\title{
Interconnection among Academic Journal Websites: Multilateral versus Bilateral Interconnection*
}

\author{
Doh-Shin Jeon ${ }^{\dagger}$ and Domenico Menicucci ${ }^{\ddagger}$
}

October 30, 2009

\begin{abstract}
Electronic academic journal websites provide new services of text and/or data mining and linking, indispensable for efficient allocation of attention among abundant sources of scientific information. Fully realizing the benefit of these services requires interconnection among websites. Motivated by CrossRef, a multilateral citation linking backbone, this paper performs a comparison between multilateral interconnection through an open platform and bilateral interconnection, and finds that publishers are fully interconnected in the former regime while they can be partially interconnected in the latter regime for exclusion or differentiation motives. Surprisingly, if partial interconnection arises for differentiation motive, exclusion of small publisher(s) occurs more often under multilateral interconnection. We also find that in the case of multilateral interconnection, a for-profit platform induces less exclusion than an open platform. Various other extensions are analyzed.
\end{abstract}

Key Words: Multilateral Interconnection, Bilateral Interconnection, Academic Journals, Internet, Open Platform, For-profit platform

JEL Code: D4, K21, L41, L82

${ }^{*}$ We thank the participants of seminars at CEMFI, IESE, Northwestern University, University of Bologna, University of Toulouse, and of the presentation at EARIE07. We also thank Guillermo Caruana, Jay Pil Choi, Giulio Federico, Chaim Fershtman (the editor), Michel Le Breton, Jin Li, Angel López, José Luis Moraga-González, Patrick Rey, Françoise Vandooren and two anonymous referees for helpful comments. Jeon gratefully acknowledges the financial support from the Spanish government under SEJ2006-09993/ECON and Ramon y Cajal grant.

${ }^{\dagger}$ Toulouse School of Economics, IESE (SP-SP) and CEPR. dohshin.jeon@gmail.com

‡Università degli Studi di Firenze, Italy. domenico.menicucci@dmd.unifi.it 
"A wealth of information creates a poverty of attention and a need to allocate that attention efficiently among the overabundance of information sources that might consume it" (Simon 1971, p. 40-41).

\section{Introduction}

Electronic publishing has been bringing fundamental changes in the market for academic journals. It has induced publishers to switch from no bundling and no price discrimination to bundling ${ }^{1}$ and price discrimination based on usage ${ }^{2}$ (McCabe, 2004). Furthermore, digitalization of text, data and image is transforming the system of academic communication into an interactive one based on the new techniques of text, data and image mining and linking. ${ }^{3}$ These techniques are extremely useful for the effective dissemination of scientific knowledge as the volume of scientific information grows exponentially. For instance, in biology where large amount of data are accumulating on genes, proteins, etc., it is all but impossible for a single researcher to keep pace with new information about just a handful of genes even though he or she has access to information about 30000 genes. The techniques help researchers to make efficient allocation of their attention among the abundant information sources by allowing them to extract meaning from digitized text and data and to search for the relevant information. The techniques are useful not only in data-rich hard sciences but also have many applications in social sciences and humanities. ${ }^{4}$

Fully realizing the benefit from techniques of text and data mining and linking requires interconnection (i.e., interoperability) among different websites of scholarly publications such that seamless cross-website search and navigation can be made. In this paper, we study publishers' incentive to interconnect their journal websites. Although we focus on the market for academic journals, our model can be applied more generally to situations in which interconnection among competing firms or information depositories generates additional value.

Actually, a large number of publishers provide links through CrossRef, a not-for-profit backbone offering a collaborative reference linking service that allows users to click on a

\footnotetext{
${ }^{1}$ Large publishers engage in direct negotiations with each library (or a consortium of libraries), in which they practice bundling and propose prices based on the individual characteristics of the library. This practice is called "Big Deal": see Edlin-Rubinfeld (2004) for more details.

${ }^{2}$ For instance, Derk Haank (2001), the CEO of Elsevier Science, says: "What we are basically doing is to say that you pay depending on how useful the publication is for you-estimated by how often you use it." See also Bolman (2002) and Key Perspectives (2002) with regard to price discrimination.

${ }^{3}$ For instance, in biology there is a software which can recognize a two-dimensional image of a molecule and search for all the articles studying the same molecule.

${ }^{4}$ For instance, "digitized corpus can be analyzed in ways scholars whose work is confined to printed volumes are not able to explore." (European Commission, 2007, p.15)
} 
citation and be taken directly to the target content. It currently has over 2,427 participating publishers and societies ${ }^{5}$ and more than 25 million content items are registered in the CrossRef system. ${ }^{6}$ In particular, it allows publishers to avoid bilateral linking agreements since a single agreement with CrossRef serves as a linking agreement with all participating publishers.

Motivated by the practice of CrossRef, this paper performs a comparison between interconnection through a multilateral platform and bilateral interconnection in terms of publishers' incentives to interconnect, profits and social welfare. In the case of multilateral interconnection, we distinguish an open platform from a for-profit platform. An open platform is defined as the one that provides interconnection service at cost. For instance, CrossRef is a not-for-profit organization and charges nominal interconnection fees. ${ }^{7}$ Another example is OpenSocial, which is application programming interfaces (APIs) for web-based social network applications, developed by Google along with MySpace. Applications implementing the OpenSocial APIs are interoperable with any social network system that supports them. OpenSocial is managed by a not-for-profit corporation created to sustain free and open development of OpenSocial specifications. ${ }^{8}$

As the main result, we find that interconnection through an open multilateral platform leads to full interconnection while bilateral interconnections often lead to partial interconnection. However, the welfare implication of the result depends on whether publishers are symmetric or not. In the case of symmetric publishers, not surprisingly, interconnection through an open multilateral platform leads to higher social welfare than bilateral interconnections. However, in the case of asymmetric publishers, which is the main focus of our paper, the comparison is ambiguous since an open platform for multilateral interconnection can lead to exclusion of small publisher more or less often than bilateral interconnections depending on whether connectivity breakdown in bilateral interconnections occurs for an exclusion motive or a differentiation motive. Furthermore, we find that a for-profit platform for multilateral interconnection gives higher social welfare than an open platform because the former induces exclusion less often than the latter.

Regarding interconnection among electronic academic journals, the U.K. Competition Commission (2001) mentions big publishers' incentive not to provide links to other pub-

\footnotetext{
${ }^{5}$ The Board of Directors currently comprises representatives from AAAS (Science), Elsevier, IEEE, John Wiley \& Sons, Nature, Sage, Springer, University of California Press etc. See http://www.crossref.org/01company/05board.html (accessed September 10, 2009).

${ }^{6}$ The statistics cited are from the annual report 2006-07 at http://www.crossref.org/07annual/exec_letter.html

${ }^{7}$ Concerning the fees charged by CrossRef, see http://www.crossref.org/02publishers/20pub_fees.html.

${ }^{8}$ For more information, see http://sites.google.com/a/opensocial.org/opensocial/opensocialfoundation
} 
lishers' websites in its report about the merger between Reed Elsevier (RE) and Harcourt: ${ }^{9}$

"... we had received some expressions of concerns from others in the industry that RE might try to undermine its competitors by denying them links with ScienceDirect, ...(p.22)".

The report on the market for academic journals commissioned by European Commission, Dewatripont et al. (2006), devotes a section to the issue of interoperability and recommends to foster interoperability by supporting research and development. To our knowledge, no paper has studied interconnection among academic journals in a formal model.

Interestingly, academic journals differ from other typical networks (such as mobile phone, Internet access, ATM cards) in their multi-homing nature. More precisely, a user must get access to both publishers' websites in order to enjoy the benefit from seamless navigation across them while in the case of mobile phone networks, for instance, it is enough to subscribe to one of them to benefit from interconnection. There are other industries such as railroads (for instance, Eurail), ski resorts, airlines etc. that share the features of the academic journal industry in that facilitating navigation across networks owned by different firms generates significant value to consumers. Furthermore, the anticompetitive issue related to refusal to provide links to rivals in academic journals is similar to the one in the well-known case, Aspen Skiing Company v. Aspen Highlands Skiing Company, 472 U.S. 585 (1985), in which the former, owning three among all four ski resorts in Aspen, refused to market all-Aspen ticket in order to weaken the competitive position of the latter, who owned only one resort.

We study games of interconnection and pricing played by competing publishers. To model price competition among publishers, we build on the common agency framework of our previous work, Jeon and Menicucci (2006): assuming price discrimination based on usage (and budget), we consider a situation in which for-profit publishers owning different portfolio of journals compete to sell them to a library ${ }^{10}$ which faces a budget constraint. Since we know from Jeon and Menicucci (2006) that each publisher has an incentive to bundle his journals, we assume, without loss of generality, that all publishers practice bundling.

In our model, there are three publishers (a large one, a middle one and a small one) and each publisher competes for relative standing as in a Hotelling model since the industry profit is equal to the budget of the library, which is given. We assume that the value created by interconnection of any pair of bundles of journals exhibits economies of scale

\footnotetext{
${ }^{9}$ At the time of the merger, RE's ScienceDirect was the most developed website and offered access to around 1,150 journals and Harcourt's IDEAL offered access to 320 journals.

${ }^{10}$ In section 7 we extend part of our analysis to the case of any number of heterogeneous libraries.
} 
and increases with the stand-alone value of each bundle in the pair. ${ }^{11}$ This implies that full interconnection among all publishers improves the relative standing of the large publisher and worsens that of the small one.

Since interconnection among firms creates a coordination problem, multiple equilibria are prevalent. We apply Coalition-Proof Nash Equilibrium (CPNE), ${ }^{12}$ introduced by Bernheim, Peleg and Whinston (1987), to interconnection games and obtain either a unique equilibrium or a unique equilibrium outcome.

When the budget is large enough that all publishers remain active regardless of interconnection profiles, we find that each publisher chooses to interconnect with all rival publishers regardless of the mode of interconnection. This is because interconnection strictly improves one's relative standing compared to no interconnection. However, if the small publisher can be excluded depending on interconnection profiles, this might affect larger publishers' incentive to interconnect.

In the case of multilateral interconnection through an open platform, we find that the possibility of exclusion does not affect incentives to interconnect and all active publishers are fully interconnected. Note first that since full interconnection among all publishers weakens the small publisher's relative standing, the latter is excluded more often than in the absence of interconnection. If the large publisher (publisher 1), for instance, does not interconnect, both publishers 2 and 3 respond by interconnecting through the platform, which weakens 1's relative standing while improving the rivals' standing.

Under bilateral interconnections, two publishers are interconnected if each of them chooses to interconnect with the other. We find that connectivity breakdown may arise for two different motives: exclusion motive or differentiation motive. First, when the big and the middle publishers are similar, in order to exclude the small one, the large one (respectively, the middle one) may break connectivity with the small one while maintaining connectivity with the middle one (respectively, the large one). In this case, the exclusion of the small one occurs more often under bilateral interconnections than under multilateral interconnection. Second, when the big one is much larger than the middle one, the big one may break connectivity with the middle one while maintaining it with the small one. This strategy allows the big one to improve its relative standing with respect to the middle one (i.e., to further differentiate itself from the middle one) since its gain from interconnecting with the small one is much larger than the middle one's gain. Then, the middle one responds by maintaining connectivity with the small one. Therefore, surprisingly, a star

\footnotetext{
${ }^{11}$ This assumption is standard in the literature: the value of interconnection increases with the size of the interconnected networks. Furthermore, in section 2, we give a microfoundation to this assumption.

${ }^{12} \mathrm{CPNE}$ is used also by Dutta and Mutuswami (1997) and by Dutta, van den Nouweland and Tijs (1998) in link announcement games a la Myerson (1991), and by Matutes and Padilla (1994) in the context of ATM networks.
} 
network emerges with the small publisher at its center. This star network minimizes the exclusion possibility of the small publisher.

From social welfare point of view, the open multilateral interconnection dominates bilateral interconnections as long as the former does not lead to exclusion of the small publisher (i.e. when the library's budget is large enough). Otherwise, bilateral interconnections dominate the open multilateral interconnection and strictly so if the small publisher becomes the center of a star network.

We also consider a hybrid interconnection game in which publishers can interconnect both bilaterally and multilaterally (through an open platform) and use this framework to study the large publisher's incentive to introduce an open multilateral platform as was the case with CrossRef. We find that this market provision of an open platform improves welfare either by reducing exclusion or by expanding interconnection.

We also investigate multilateral interconnection through a monopoly for-profit platform. We characterize the profit-maximizing fees and the resulting interconnection profile using CPNE as a solution concept. We find that a for-profit platform generates higher welfare than an open platform. If an open platform does not generate any exclusion, the two platforms are equivalent in terms of social welfare; otherwise, a for-profit platform generates less exclusion. Given that benefits from interconnections exhibit economies of scale, free access to interconnection technology through an open platform amplifies the existing asymmetry to the detriment of the small publisher. On the contrary, a monopoly for-profit platform may have an incentive to discriminate publishers in favor of the small one in order to gather fees, especially when no interconnection status quo leads to exclusion of the small one.

Finally, in the case of symmetric publishers, we find the same result that multilateral interconnection through an open platform leads to full interconnection while bilateral interconnections can lead to partial interconnection (and thereby exclusion of a publisher). This result implies that the open multilateral interconnection dominates bilateral interconnection from social welfare point of view.

Interconnection (or compatibility) choice among firms in network industries has been a subject of intensive investigation in IO literature. ${ }^{13}$ The seminal papers on economics of network externalities (Farrell and Saloner 1985, 1986, Katz and Shapiro, 1985) study the compatibility issue. For instance, Katz and Shapiro (1985) show that a dominant firm may choose to remain incompatible with a rival because it will suffer a substantial decline in market share if it becomes compatible. ${ }^{14}$ More recently, the literature on two-way

\footnotetext{
${ }^{13}$ See for instance Church and Gandal (2005) for a recent survey.

${ }^{14}$ Farrell and Saloner $(1985,1986)$ examine a dynamic issue of how network externalities affect the adoption of a new technology and identify inefficiency in terms of both "excess momentum" and "excess inertia".
} 
access pricing among telecommunication networks, initiated by Armstrong (1998) and Laffont-Rey-Tirole (1998a,b), studies how access prices affect retail competition and interconnection through telecommunications networks' choice of retail tariffs. In addition, Crémer-Rey-Tirole (2000) and Laffont-Marcus-Rey-Tirole (2003) study interconnection among Internet Backbone providers in a peering regime or in a regime of access pricing respectively. ${ }^{15} \mathrm{~A}$ general finding in the literature on interconnection without mediation through access prices is that when networks are asymmetric, big networks might have an incentive to make the networks incompatible since complete compatibility means that big and small networks become equal (Katz-Shapiro, 1985, Crémer-Rey-Tirole, 2000). We contribute to the literature by studying interconnection through an open (and a for-profit) multilateral platform and comparing it with bilateral interconnection: the existing literature on interconnection typically considers two firms and hence does not make distinction between the two modes of interconnection. ${ }^{16}$

Our bilateral interconnection game is closely related to the literature on strategic network formation (Myerson, 1991, Jackson and Wolinsky 1996, see, for survey, Goyal, 2007 and Jackson, 2008). ${ }^{17}$ Although it is standard in this literature to use pairwise stability (Jackson and Wolinsky 1996) as a refinement for Nash Equilibria, we use CPNE since we find that in some cases there are multiple pairwise stable Nash equilibria. The papers closest to ours in the literature are Goyal and Moraga-Gonzales (2001) and Goyal, Moraga-Gonzales and Konovalov (2008) in that they study games in which a network formation stage is followed by a price or quantity competition stage. Both papers study formation of $R \& D$ networks when each firm chooses the amount of cost-reducing $R \& D$ investments after network formation.

The rest of the paper is organized as follows. Section 2 describes the model. Section 3 analyzes the pricing game given any interconnection profile. Section 4 defines CPNE and studies multilateral interconnection through an open platform and bilateral interconnection. Section 5 studies the hybrid interconnection game and market provision of an open platform. Section 6 studies multilateral interconnection through a for-profit platform. Section 7 extends the analysis of open multilateral interconnection to multiple heterogeneous libraries. Section 8 studies symmetric publishers. Section 9 provides social welfare

\footnotetext{
${ }^{15}$ See Economides (2006) for a survey on the economics of the Internet Backbone market.

${ }^{16}$ To our knowledge, Matutes-Padilla (1994) and Crémer-Rey-Tirole (2000) are the only papers that consider more than two firms. However, none of them makes a distinction between a multilateral and a bilateral interconnections.

${ }^{17}$ Our multilateral interconnection game is similar to the open membership game (Yi 1998, Belleflamme, 2000, Yi and Shin 2000), in which each player announces an address and the players choosing the same address become members of a coalition. If we assume that there is a unique feasible address and each player chooses between the address and no address, then we obtain our multilateral interconnection game. Furthermore, Belleflamme (2000) and Yi and Shin (2000) use CPNE.
} 
analysis. Section 10 concludes the paper. For the sake of brevity, the appendix presents the proofs of only some of our results, while the complete proofs are found in Jeon and Menicucci (2009).

\section{Model: Moves, information, preferences}

We consider a game with three publishers and a library; publisher $i$ is often simply denoted by $i$, for $i=1,2,3$. We consider profit-maximizing publishers and assume that they practice pure bundling ${ }^{18}$ and price discrimination based on usage and budget; $\mathrm{B}_{i}$ denotes $i$ 's bundle. Since our analysis of the bilateral interconnection is complicated even with one library, we consider the case of a single library. However, we extend the analysis of an open multilateral interconnection to any number of heterogenous libraries (see section 7). Note that since publishers practice price discrimination based on usage and budget, considering only one library does not involve loss of generality at the pricing stage.

In this section, we introduce the games that we analyze.

\subsection{Games with and without interconnection}

We study two games of interconnection, which differ depending on whether the interconnection regime is multilateral or bilateral. In the case of multilateral interconnection, we study in most sections interconnection through an open platform such as CrossRef and analyze in section 6 interconnection through a for-profit platform. We here introduce the game of multilateral interconnection through an open platform denoted by $\Gamma^{m}$, the game of bilateral interconnection denoted by $\Gamma^{b}$ and, as a benchmark, the game without interconnection denoted by $\Gamma^{0}$.

We first describe $\Gamma^{m}$.

- (interconnection) In stage one, each publisher $i$ (for $i=1,2,3$ ) simultaneously decides whether or not to interconnect through CrossRef; we let $x_{i}=1$ if he ${ }^{19}$ has joined CrossRef, $x_{i}=0$ otherwise.

- (active/non-active) In stage two, after the moves at stage one have been observed by all publishers and the library, each publisher simultaneously decides whether to be active or not; we use $A \subseteq\{1,2,3\}$ to denote the set of active publishers.

\footnotetext{
${ }^{18}$ We can mimic the arguments in the proof of Proposition 2(i) in Jeon and Menicucci (2006) to show that, for each publisher, bundling the journals weakly dominates any alternative to pure bundling, such as independent sales (for instance).

${ }^{19}$ We use "he" for a publisher.
} 
- (pricing) In stage three, after the moves at stage two have been observed by all publishers and the library, each publisher $i \in A$ simultaneously chooses a price $P_{i} \geq 0$ for $\mathrm{B}_{i}$.

- In stage four, the library chooses bundles to buy from the set $\left\{\mathrm{B}_{i}: i \in A\right\}$.

In this game, by joining CrossRef a publisher makes multiple interconnections, i.e. with all the other publishers who are members of CrossRef. Conversely, if publisher $i$ does not join CrossRef then he is not interconnected with any other publisher. We use $y \equiv\left(y_{12}, y_{13}, y_{23}\right)$ to represent the interconnection profile by defining $y_{12} \equiv x_{1} x_{2}$, $y_{13} \equiv x_{1} x_{3}$ and $y_{23} \equiv x_{2} x_{3}$. Clearly, $y_{i j}=0$ or $y_{i j}=1$, and $y_{i j}=1$ means that $i$ and $j$ are interconnected, while $y_{i j}=0$ means there is no interconnection between them.

In $\Gamma^{b}$, publisher $i$ can refuse to interconnect with publisher $j$ while being interconnected with $k(\neq j)$. Formally, $\Gamma^{b}$ is such that:

- (interconnection) In stage one, each publisher $i$ (for $i=1,2,3$ ) simultaneously chooses $x_{i j} \in\{0,1\}$ and $x_{i k} \in\{0,1\}$ which denote his willingness to interconnect with publisher $j$ and with publisher $k(\neq j)$, respectively.

Stages two, three and four are like in $\Gamma^{m}$.

In $\Gamma^{b}$, the variables in $y \equiv\left(y_{12}, y_{13}, y_{23}\right)$ are defined as follows: $y_{12} \equiv x_{12} x_{21}, y_{13} \equiv$ $x_{13} x_{31}$ and $y_{23} \equiv x_{23} x_{32}$. In $\Gamma^{b}$, for instance, publishers 1 and 2 can interconnect among themselves (i.e., $x_{12}=x_{21}=1$ ) and at the same time 1 (2) can break connectivity with 3 by choosing $x_{13}=0\left(x_{23}=0\right)$. In contrast, in $\Gamma^{m}$, in order for 1 and 2 to be interconnected, it is necessary that $x_{1}=x_{2}=1$, and then 3 can interconnect with both 1 and 2 by choosing $x_{3}=1$. Another interconnection configuration of interest that does not exist in $\Gamma^{m}$ but can exist in $\Gamma^{b}$, for instance, is such that 3 is interconnected with both 1 and 2 but 1 and 2 are not interconnected between themselves. This occurs if $\left(x_{12}, x_{13}\right)=(0,1),\left(x_{21}, x_{23}\right)=(0,1)$ and $\left(x_{31}, x_{32}\right)=(1,1)$, so that $y=(0,1,1)$.

In order to isolate the effects of interconnection, we first analyze (in section 3 ) as a benchmark the game $\Gamma^{0}$ in which interconnection is infeasible. This means that in $\Gamma^{0}$ there is no stage one and the game starts with stage two; stages two-four are like in $\Gamma^{m}$. We use $\Gamma$ to denote an unspecified game in $\left\{\Gamma^{m}, \Gamma^{b}, \Gamma^{0}\right\}$ and for any $\Gamma$ we let $\gamma_{I I I}$ represent the subgame of $\Gamma$ which starts in stage three, in which the active publishers choose prices for their bundles and then the library makes her purchases. Clearly, $\gamma_{I I I}$ depends on the active publishers and on the interconnection profile $y$, but we do not emphasize this fact in the notation. Likewise, we use $\gamma_{I I}$ to denote the subgame of $\Gamma$ which starts in stage two, in which (given $y$ ) each publisher chooses whether to be active or not and then $\gamma_{I I I}$ starts. 


\subsection{The agents' preferences}

In order to determine the library's purchases in stage four it is necessary to specify the library's preferences. A publisher's website provides several services, which can be regrouped into two categories: basic service (reading, printing and downloading articles) and advanced service (text and data mining and linking). Let $b_{i}$ represent the gross utility that the library obtains from basic service after buying $\mathrm{B}_{i}$. For most sections, we consider asymmetric publishers with $b_{1}>b_{2}>b_{3}>0$. The library's utility from basic service when it purchases $\mathrm{B}_{i} \& \mathrm{~B}_{j}$ or $\mathrm{B}_{1} \& \mathrm{~B}_{2} \& \mathrm{~B}_{3}$ is $b_{i}+b_{j}$ or $b_{1}+b_{2}+b_{3}$, respectively.

The library's utility from advanced service depends both on the bundles it buys and on the interconnection profile among them. In case the library subscribes only to $\mathrm{B}_{i}$, its utility from advanced service is $I\left(b_{i}\right)$, where $I(0)=0$ and $I($.$) is assumed to be strictly$ increasing and strictly convex (for instance, $I(b)=\alpha b^{2}$ with $\alpha>0$ ); thus, the marginal surplus from advanced service increases as $b_{i}$ becomes larger. If the library purchases $\mathrm{B}_{i} \& \mathrm{~B}_{j}$, its utility from advanced service is $I\left(b_{i}+b_{j}\right)$ if $y_{i j}=1,{ }^{20}$ is $I\left(b_{i}\right)+I\left(b_{j}\right)$ if $y_{i j}=0$. Notice that $I\left(b_{i}+b_{j}\right)>I\left(b_{i}\right)+I\left(b_{j}\right)$ since $I($.$) is strictly convex; hence, interconnection$ between two websites creates a positive value for the library. ${ }^{21}$ Finally, if the library buys $\mathrm{B}_{1} \& \mathrm{~B}_{2} \& \mathrm{~B}_{3}$, its utility from advanced service is determined as follows: ${ }^{22}$

$\begin{aligned} & \text { library's utility from advanced } \\ & \text { service if it buys } \mathrm{B}_{1} \& \mathrm{~B}_{2} \& \mathrm{~B}_{3}{ }^{23}\end{aligned}=\left\{\begin{array}{cc}I\left(b_{1}\right)+I\left(b_{2}\right)+I\left(b_{3}\right) & \text { if } y_{12}=y_{13}=y_{23}=0 \\ I\left(b_{i}+b_{j}\right)+I\left(b_{k}\right) & \text { if } y_{i j}=1 \text { and } y_{i k}=y_{j k}=0 \\ I\left(b_{i}+b_{j}\right)+I\left(b_{i}+b_{k}\right)-I\left(b_{i}\right) & \text { if } y_{i j}=y_{i k}=1 \text { and } y_{j k}=0 \\ I\left(b_{1}+b_{2}+b_{3}\right) & \text { if } y_{12}=y_{13}=y_{23}=1\end{array}\right.$

In order to simplify notation, we introduce $U_{i} \equiv b_{i}+I\left(b_{i}\right), \mathbf{U} \equiv\left(U_{1}, U_{2}, U_{3}\right), U \equiv$ $U_{1}+U_{2}+U_{3}, I_{i j} \equiv I\left(b_{i}+b_{j}\right)-I\left(b_{i}\right)-I\left(b_{j}\right)>0$ and $\mathbf{I} \equiv\left(I_{12}, I_{13}, I_{23}\right)$. While $U_{i}$ represents the "stand-alone" utility that the library obtains from $\mathrm{B}_{i}$ when $y_{i j}=y_{i k}=0, I_{i j}$ is the increase in surplus for the library, with respect to $U_{i}+U_{j}$, from interconnection between $i$ and $j$. The assumption $b_{1}>b_{2}>b_{3}$ implies $U_{1}>U_{2}>U_{3}$ and $I_{12}>I_{13}>I_{23}$.

The library's total utility from buying one or more bundles is given by its utility from basic service plus the utility from advanced service, minus the money spent. We assume

\footnotetext{
${ }^{20}$ We may also add a parameter representing the quality of interconnection, but it would not affect our results while adding notational complexity.

${ }^{21}$ The value added by interconnection would be zero if $I($.$) were linear (i.e., if I(b)=\alpha b$ ) and would be negative if $I($.$) were strictly concave. Therefore, we view convexity of I($.$) as a reasonable assumption.$

${ }^{22} \mathrm{By}$ the strictly convexity of $I($.$) , this utility increases as the number of interconnected websites$ increases. We prove below, however, that the equilibrium prices are determined only by the library's payoff when it buys one or two bundle(s), and not by its payoff when it buys $\mathrm{B}_{1} \& \mathrm{~B}_{2} \& \mathrm{~B}_{3}$.

${ }^{23}$ For instance, when $I(b)=\alpha b^{2}$ with $\alpha>0$, we have: $I\left(b_{1}\right)+I\left(b_{2}\right)+I\left(b_{3}\right)=\alpha\left[\left(b_{1}\right)^{2}+\left(b_{2}\right)^{2}+\left(b_{3}\right)^{2}\right]$, $I\left(b_{i}+b_{j}\right)+I\left(b_{k}\right)=I\left(b_{1}\right)+I\left(b_{2}\right)+I\left(b_{3}\right)+2 \alpha b_{i} b_{j}, I\left(b_{i}+b_{j}\right)+I\left(b_{i}+b_{k}\right)-I\left(b_{i}\right)=I\left(b_{1}\right)+I\left(b_{2}\right)+I\left(b_{3}\right)+$ $2 \alpha b_{i} b_{j}+2 \alpha b_{i} b_{k}, I\left(b_{1}+b_{2}+b_{3}\right)=I\left(b_{1}\right)+I\left(b_{2}\right)+I\left(b_{3}\right)+2 \alpha b_{i} b_{j}+2 \alpha b_{i} b_{k}+2 \alpha b_{j} b_{k}$.
} 
that the library has a fixed budget $M>0$ which can be used only to buy journals. ${ }^{24}$ Therefore, publishers compete for the library's budget and we assume that they have complete information about $(M, \mathbf{U}, \mathbf{I})$.

If we assume $M \geq U$, then we find that in $\Gamma^{0}$ (the benchmark without interconnection) there is no competition among publishers since publisher $i=1,2,3$ can extract the library's full surplus from $\mathrm{B}_{i}$ by charging a price equal to $U_{i}$. While this fact suggests to restrict attention to $M<U$, we actually assume $M \leq U-I_{12}$, which is a sufficient condition to remove the multiplicity of equilibrium prices in $\gamma_{I I I}$ that arises because of interconnection. ${ }^{25}$ When we study asymmetric publishers, the two following assumptions play an important role.

A1: $I($.$) is not too convex such that the following inequalities hold: U_{1} \geq U_{2}+I_{23}$, $U_{2} \geq U_{3}+I_{13}, U_{3} \geq I_{12}$.

$\mathbf{A 1}^{w}: I($.$) is not too convex such that the following inequalities hold: U_{2}+I_{23} \geq U_{3}+I_{13}$, $U_{3} \geq I_{12}$.

The inequalities $U_{1} \geq U_{2}+I_{23}$ and $U_{2} \geq U_{3}+I_{13}$ in A1 imply that interconnection does not alter the ranking of profits among the publishers with respect to the benchmark of no interconnection. This is a simplifying assumption that allows us to focus on publisher 3's decision to be active or not when we study how interconnection affects the set of active publishers. However, when we consider multilateral interconnection we find that the game is simple enough in terms of strategy spaces and of possible outcomes that the same results obtained under A1 hold even under the weaker assumption $\mathrm{A} 1^{w}$ where the superscript $w$ means "weak". The inequality $U_{2}+I_{23} \geq U_{3}+I_{13}$ rules out that only 1 and 3 are active when publishers are fully interconnected, as it guarantees that 2 can profitably become active. Since the analysis under A1 is considerably simpler than under $\mathrm{A} 1^{w}$, all the proofs in this paper rely on $\mathrm{A} 1$, while the proofs under $\mathrm{A} 1^{w}$ can be found in Jeon and Menicucci (2009). ${ }^{26}$ In order to check the robustness of our results derived from asymmetric publishers, we consider symmetric publishers (i.e., $U_{1}=U_{2}=U_{3}$ ) in section 8 .

\footnotetext{
${ }^{24}$ In Jeon and Menicucci (2006) we allow the library to use the budget to buy journals and books and assume that the library obtains utility $v(m)$ from spending $m \leq M$ to purchase books, with $v$ increasing and concave. Hence, we can see our current setting as one in which $v(m)=m$. Since interconnection complicates the analysis, we make this simplification to obtain closed form formulas for equilibrium prices.

${ }^{25}$ Two interconnected publishers sell complementary products and this creates multiple equilibrium prices in $\gamma_{I I I}$ if $M>U-I_{12}$ holds.

${ }^{26}$ The inequality $U_{3} \geq I_{12}$ implies that the value from interconnection is not large relative to the value from the original bundle of journals. This simplifies the statements of our results but does not affect the results themselves. For instance, if $I_{12}>2 U_{2}+U_{3}$ then $U-I_{12}$ is smaller than $U_{1}-U_{2}$ and sometimes this implies that only 1 is active for any $M$ that we consider. This case is not interesting since interconnection plays no role.
} 
Since we would like to study publishers' incentives to interconnect and how interconnection affects which publishers succeed in selling their bundles and at what prices, we assume that the fixed cost of having the first electronic copy of each journal has already been incurred. We also assume that the marginal cost of distributing each electronic journal through Internet to the library is zero. Furthermore, we assume that providing interconnection is costless and hence an open multilateral platform charges zero interconnection fee. This simplifying assumption implies that the profit of an active publisher $i$ is equal to $P_{i}$ if the library purchases $\mathrm{B}_{i}$, while $i$ 's profit is 0 otherwise, and thus we can focus on strategic interconnection incentives related to journal pricing. ${ }^{27}$ However, for comparison with an open platform, in section 6 we study a for-profit platform for multilateral interconnection which charges interconnection fees.

In the next sections we use the concept of subgame perfect Nash equilibrium (SPNE henceforth) to determine the publishers' behavior in $\Gamma$. For this purpose, we apply backward induction and thus we need to find Nash equilibrium (NE henceforth) of $\gamma_{I I I}$ under various possible scenarios regarding the interconnection profile and the set of active firms. However, when all three publishers are active, in some cases $\gamma_{I I I}$ has infinitely many NEs in which the prices of the bundles the library buys depend on the prices of bundles the library does not buy. ${ }^{28}$ In order to eliminate this indeterminacy, we adopt the following tie-breaking rule as in Jeon and Menicucci (2006).

T1: In each game $\Gamma \in\left\{\Gamma^{m}, \Gamma^{b}, \Gamma^{0}\right\}$, any publisher $i$ prefers being non-active to being active but unable to make a strictly positive profit.

T1 can be justified if a publisher would incur a very small but positive cost for contracting with the library. Therefore, in a SPNE, publisher $i$ is active if and only if in stage four the library purchases $\mathrm{B}_{i}$ at some price $P_{i}>0$.

In what follows, we let $P_{i}^{*}$ denote the equilibrium price for $\mathrm{B}_{i}$ and, likewise, $\left(x_{i}^{*}, x_{i j}^{*}, y_{i j}^{*}, A^{*}\right)$ represents $\left(x_{i}, x_{i j}, y_{i j}, A\right)$ in equilibrium. For each $\Gamma \in\left\{\Gamma^{m}, \Gamma^{b}, \Gamma^{0}\right\}$, under A1 an intuitive result holds and can be stated as follows: in any SPNE, publisher 1 is active; if publisher 3 is active, then publisher 2 is active as well. In other words, the following lemma applies.

Lemma 1 For any interconnection profile y and any interconnection game $\Gamma \in\left\{\Gamma^{m}, \Gamma^{b}, \Gamma^{0}\right\}$, A1 implies that any SPNE of $\gamma_{I I}$ is such that the set of active publishers $A^{*}$ is either $\{1\}$ or $\{1,2\}$ or $\{1,2,3\}$.

\footnotetext{
${ }^{27}$ We remark, however, that our results qualitatively hold if we introduce a small positive interconnection fee.

${ }^{28}$ For instance, suppose that interconnection is infeasible and $U_{1}=10, U_{2}=2, U_{3}=1$ and $M=9$. Then, for any $\beta \in\left[0, \frac{2}{3}\right]$, there exists a NE of $\gamma_{I I I}$ in which $P_{1}=9-\beta, P_{2}=P_{3}=\beta$ and the library buys $\mathrm{B}_{1} \& \mathrm{~B}_{2}$.
} 


\section{Subgame for given interconnection profile}

In this section, we first describe the unique SPNE of the subgame $\gamma_{I I}$ that starts in stage two as a function of the interconnection profile and then describe the unique SPNE of the game without interconnection $\Gamma^{0}$.

\subsection{Subgame for given interconnection profile}

In order to apply backward induction to $\gamma_{I I}$, we start by studying the NE of the pricing game $\gamma_{I I I}$ that starts in stage three. Namely, the next lemma considers the cases of $A=\{1,2,3\}$ and $A=\{1,2\}$, in view of Lemma 1 , and provides the conditions on $M$ under which all active publishers realize positive profits; it also characterizes the equilibrium prices.

Lemma 2 (i) Under A1, let the set of active publishers be $A=\{1,2,3\}$. Then, for any given interconnection profile $y$, there exists a NE of the pricing game $\left(\gamma_{I I I}\right)$ in which the library buys all three bundles and all publishers realize strictly positive profits if and only if $M>M^{t}(y) \equiv U-3 U_{3}+2 I_{12} y_{12}-I_{13} y_{13}-I_{23} y_{23}$. Furthermore, for any $M$ between $M^{t}(y)$ and $U-I_{12}$, the $N E$ is unique and prices are given by:

$$
\begin{aligned}
& P_{1}^{*}=P_{1}^{t}(y) \equiv U_{1}+\frac{1}{3}\left(M-U+\bar{I}-3 I_{23} y_{23}\right), \\
& P_{2}^{*}=P_{2}^{t}(y) \equiv U_{2}+\frac{1}{3}\left(M-U+\bar{I}-3 I_{13} y_{13}\right), \\
& P_{3}^{*}=P_{3}^{t}(y) \equiv U_{3}+\frac{1}{3}\left(M-U+\bar{I}-3 I_{12} y_{12}\right) ;
\end{aligned}
$$

where $\bar{I} \equiv I_{12} y_{12}+I_{13} y_{13}+I_{23} y_{23}$. Therefore, $P_{1}^{t}(y)+P_{2}^{t}(y)+P_{3}^{t}(y)=M$.

(ii) Let the set of active publishers be $A=\{1,2\}$. Then, independently of the interconnection between 1 and 2, there exists a NE of the pricing game $\left(\gamma_{I I I}\right)$ in which the library buys both bundles and both publishers 1 and 2 realize strictly positive profits if and only if $M>U_{1}-U_{2}$. Furthermore, for any $M$ between $U_{1}-U_{2}$ and $U_{1}+U_{2}$, the $N E$ is unique and prices are given by

$$
P_{1}^{*}=P_{1}^{d} \equiv \frac{1}{2}\left(M+U_{1}-U_{2}\right), \quad P_{2}^{*}=P_{2}^{d} \equiv \frac{1}{2}\left(M+U_{2}-U_{1}\right)
$$

Therefore, $P_{1}^{d}+P_{2}^{d}=M$.

In order to explain Lemma 2(i), we suppose that $A=\{1,2,3\}$ and study a NE in which the library buys all three bundles. The equilibrium prices $\left(P_{1}^{*}, P_{2}^{*}, P_{3}^{*}\right)$ need to be such that the library does not purchase $\mathrm{B}_{i}$ anymore if publisher $i$ increases $P_{i}$ above $P_{i}^{*}$, and in the proof of the lemma we show that this condition is satisfied for each $i$ if and 
only if the library is indifferent between buying $\mathrm{B}_{i} \& \mathrm{~B}_{j}$ and buying $\mathrm{B}_{i} \& \mathrm{~B}_{k}$, for any $i, j, k$. This is equivalent to the following equalities

$$
\begin{aligned}
& U_{1}+U_{2}+I_{12} y_{12}-P_{1}^{*}-P_{2}^{*}=U_{1}+U_{3}+I_{13} y_{13}-P_{1}^{*}-P_{3}^{*} \\
& U_{1}+U_{2}+I_{12} y_{12}-P_{1}^{*}-P_{2}^{*}=U_{2}+U_{3}+I_{23} y_{23}-P_{2}^{*}-P_{3}^{*} .
\end{aligned}
$$

Furthermore, the assumption $M<U-I_{12}$ implies $P_{1}^{*}+P_{2}^{*}+P_{3}^{*}=M$, and using this equality ${ }^{29}$ jointly with (1)-(2) we find $\left(P_{1}^{*}, P_{2}^{*}, P_{3}^{*}\right)=\left(P_{1}^{t}(y), P_{2}^{t}(y), P_{3}^{t}(y)\right)^{30}$ as in Lemma 2(i). It is readily seen that $P_{i}^{t}(y)$ is increasing in $y_{i j}$ and $y_{i k}$ and decreasing in $y_{j k}$, so that publisher $i$ 's profit is positively affected by his own interconnection with other publishers, but is negatively affected if his rivals are interconnected. The reason is that the library's payoff from buying $\mathrm{B}_{i} \& \mathrm{~B}_{j}$ (for instance) is higher when $y_{i j}=1$ than when $y_{i j}=0$ and therefore, after interconnection between $i$ and $j, P_{i}$ needs to be higher in order to make the library remain indifferent between $\mathrm{B}_{i} \& \mathrm{~B}_{j}$ and $\mathrm{B}_{j} \& \mathrm{~B}_{k}$.

Quite importantly, we notice that A1 implies $P_{1}^{t}(y)>P_{2}^{t}(y)>P_{3}^{t}(y)$ for any interconnection profile $y$, and $P_{3}^{t}(y)>0$ if and only if $M>M^{t}(y)$. Therefore, when $M \leq M^{t}(y)$, no NE of $\gamma_{I I I}$ exists in which all three publishers make a profit. Since $P_{3}^{t}$ is increasing in $y_{13}$ and $y_{23}$ and decreasing in $y_{12}$, it follows that $M^{t}(y)$ increases (decreases) in $y_{12}$ (in $y_{13}$ and $\left.y_{23}\right)$.

In the case that $M \leq M^{t}(y)$, either only 1 is active, or only 1 and 2 are active. Let us consider first the case in which 1 and 2 are active. Then their prices are determined by a principle similar to the one explained above: given $\left(P_{1}^{*}, P_{2}^{*}\right)$, publisher $i(i=1,2)$ has no incentive to increase $P_{i}$ above $P_{i}^{*}$ if and only if the library is indifferent between purchasing only $\mathrm{B}_{1}$ and purchasing only $\mathrm{B}_{2}$ :

$$
U_{1}-P_{1}^{*}=U_{2}-P_{2}^{*}
$$

This condition and $P_{1}^{*}+P_{2}^{*}=M$ yield $\left(P_{1}^{d}, P_{2}^{d}\right)^{31}$ and we notice that $P_{2}^{d}>0$ if and only if $M>U_{1}-U_{2}$. Therefore, when $M \leq U_{1}-U_{2}$, no NE of $\gamma_{I I I}$ exists in which both 1 and 2 make a profit. Notice that the interconnection profile has no impact on the equilibrium prices when only two publishers are active.

Finally, in the case of $M \leq U_{1}-U_{2}$ (independently of $y$ ) there is no NE of $\gamma_{I I I}$ in which both publishers 1 and 2 (or all the three publishers) make a positive profit. This implies that, when $M \leq U_{1}-U_{2}$, only 1 will be active and $P_{1}^{*}=M$. The reason is that if publisher 2 (for instance) is active as well, he cannot make any profit in $\gamma_{I I I}$ because the library's profit from buying only $\mathrm{B}_{1}$ is $U_{1}-M$, which is higher than the profit $U_{2}-P_{2}$

\footnotetext{
${ }^{29}$ If $P_{1}^{*}+P_{2}^{*}+P_{3}^{*}<M$, then $P_{i}^{*}<U_{i}$ holds for at least one $i$. As a consequence, there exists a profitable deviation for publisher $i$ : if he slightly increases $P_{i}$ above $P_{i}^{*}$, he still succeed in selling $\mathrm{B}_{i}$.

${ }^{30}$ The superscript $t$ means triopoly.

${ }^{31}$ The superscript d means duopoly.
} 
obtained by purchasing $\mathrm{B}_{2}$, for any $P_{2}>0$. When $M>U_{1}-U_{2}$, instead, this argument does not apply because $U_{2}-P_{2}>U_{1}-M$ for $P_{2}$ close to 0 ; thus $A^{*}$ will include at least $\{1,2\}$. However, whether also 3 will be active or not depends on whether $M$ is larger or smaller than $M^{t}(y)$. Thus, $y$ does not affect the set of parameter values for which only 1 is active but determines the values of $M$ for which all publishers are active.

The results of Lemma 2 help us to derive the set of active publishers as a function of $y$ as in the next lemma.

Lemma 3 Under A1, for any given interconnection profile $y$, the unique SPNE of the game starting with stage two $\left(\gamma_{I I}\right)$ is such that

(i) if $M \leq U_{1}-U_{2}$, only publisher 1 is active and charges $P_{1}^{*}=M$;

(ii) if $U_{1}-U_{2}<M \leq M^{t}(y)$, only publisher 1 and publisher 2 are active and charge $P_{1}^{d}$ and $P_{2}^{d}$;

(iii) if $M^{t}(y)<M$, all three publishers are active and charge $P_{1}^{t}(y), P_{2}^{t}(y)$ and $P_{3}^{t}(y)$.

From now on, we neglect the case of $M \leq U_{1}-U_{2}$ since then only 1 is active regardless of interconnection profile $y$. In section 4 , we use this lemma in order to examine how stage-one interconnection decisions affect the outcome of the game.

\subsection{Benchmark: The game without interconnection}

We now consider as a benchmark the game without interconnection $\Gamma^{0}$. In $\Gamma^{0}$ there is no stage one and the interconnection profile is $y=(0,0,0)$; this implies that $\Gamma^{0}$ coincides with the subgame $\gamma_{I I}$ of $\Gamma^{m}$ following $y=(0,0,0)$. The next proposition, which is just an immediate consequence of Lemma 3 , describes the unique SPNE of $\Gamma^{0}$.

Proposition 1 In the game without interconnection, there exists a unique SPNE and the equilibrium active publishers and prices are given by:

(i) if $U_{1}-U_{2}<M \leq M^{t}(0,0,0)$, then only publisher 1 and publisher 2 are active and charge $P_{i}^{*}=P_{i}^{d}$ for $i=1,2$;

(ii) if $M^{t}(0,0,0)<M \leq U-I_{12}$, then all three publishers are active and charge $P_{i}^{*}=$ $P_{i}^{t}(0,0,0)$ for $i=1,2,3$.

When there is no interconnection, Proposition 1 establishes that the library buys $\mathrm{B}_{1} \& \mathrm{~B}_{2} \& \mathrm{~B}_{3}$ if and only if $M$ is larger than $M^{t}(0,0,0)=U-3 U_{3}$; otherwise publisher 3 is excluded from the market. 


\section{The interconnection games}

In this section, we study the two interconnection games: open multilateral interconnection game $\Gamma^{m}$ and bilateral interconnection game $\Gamma^{b}$. From Lemma 3, for any interconnection profile $y$ determined in stage one, we can find each publisher's profit. Therefore, we can find the equilibrium interconnection profile by considering a one-stage game in which each publisher chooses his interconnection strategy knowing that the resulting interconnection profile generates profits as described by Lemma 3. Since this game has multiple equilibria, we first introduce a refinement concept and then study the open multilateral interconnection and the bilateral interconnection.

\subsection{Coalition-Proof Nash Equilibrium (CPNE)}

Before introducing the equilibrium refinement concept, we define a one-stage game of interconnection. Let $s_{i}$ denote publisher $i$ 's interconnection strategy: in the case of multilateral interconnection, $s_{i}=x_{i} \in\{0,1\}$ and in the case of bilateral interconnection, $s_{i}=\mathbf{x}_{i}=\left(x_{i j}, x_{i k}\right) \in\{(0,0),(0,1),(1,0),(1,1)\}$. As a function of $s=\left(s_{1}, s_{2}, s_{3}\right)$, an interconnection profile $y(s)$ is determined and the resulting profit of publisher $i$, denoted by $\pi_{i}(s)$, is obtained from Lemma 3 as follows

$$
\left\{\begin{array}{c}
\pi_{1}(s)=M, \quad \pi_{2}(s)=\pi_{3}(s)=0 \quad \text { if } \quad M \leq U_{1}-U_{2} \\
\pi_{1}(s)=P_{1}^{d}, \quad \pi_{2}(s)=P_{2}^{d}, \quad \pi_{3}(s)=0 \quad \text { if } \quad U_{1}-U_{2}<M \leq M^{t}(y(s)) \\
\pi_{i}(s)=P_{i}^{t}(y(s)) \text { for } i=1,2,3 \quad \text { if } \quad M^{t}(y(s))<M<U-I_{12}
\end{array}\right.
$$

In this section, we consider the one-stage game in which each publisher $i$ simultaneously chooses $s_{i}$ taking into account that his profit is $\pi_{i}(s)$. Let $G^{m}$ denote the one-stage game with open multilateral interconnection and $G^{b}$ be the one-stage game with bilateral interconnection.

Both $G^{m}$ and $G^{b}$ can be viewed as games of network formation, and indeed a game which is essentially equivalent to $G^{b}$ has received a lot of attention in the last few years in the literature on strategic network formation (for surveys, see Goyal, 2007, and Jackson, 2008), which has been developed independently of the literature on interconnection in network industries. Precisely, a game with the bilateral linking structure of $G^{b}$ has been proposed by Myerson (1991, page 448) under the assumption that the resulting network determines profits as a function of an underlying cooperative game. As it is apparent, the notion of NE for this game is not particularly useful: since a link between two players is formed only when both players agree, a coordination problem arises and multiple NEs exist. For instance, independently of the parameters, in our setting $\mathbf{x}_{1}=\mathbf{x}_{2}=\mathbf{x}_{3}=$ $(0,0)$ is a NE of $G^{b}$, even though there could be a pair of publishers which benefit from interconnection. As in $G^{b}$, a coordination problem arises in $G^{m}$ as well and multiple NEs 
exist (although the degree of multiplicity is less severe in $G^{m}$ than in $G^{b}$ ); for instance, $x_{1}=x_{2}=x_{3}=0$ is always a NE of $G^{m}$.

An obvious difference between $G^{m}$ and $G^{b}$ is that in $G^{b}$ any interconnection profile $y \in\{0,1\}^{3}$ is feasible, unlike in $G^{m}$. Precisely, the empty network, one-link networks and the complete network can be formed both in $G^{m}$ and in $G^{b}$. But a two-link network can arise only in $G^{b}$ when publishers play $x_{i j}=x_{j i}=1, x_{i k}=x_{k i}=1$ and $x_{j k} x_{j k}=0$; in this case the network is called a star, with publisher $i$ at the center of the star.

In order to address the multiplicity of equilibria, we apply to $G^{m}$ and $G^{b}$ the notion of Coalition-Proof Nash Equilibrium (CPNE), a refinement introduced by Bernheim, Peleg and Whinston (1987). The general definition of CPNE is recursive and hence is not straightforward, but it is simple in a three-player game like $G^{m}$ or $G^{b}$. Let $G_{i, j}^{m}\left(s_{k}\right)$ denote the game of multilateral interconnection played between publishers $i$ and $j$ in which the interconnection choice of firm $k$ (the remaining publisher) is kept fixed at a certain $s_{k}$; $G_{i, j}^{b}\left(s_{k}\right)$ is defined similarly. Then

Definition 1 A profile $s^{*}=\left(s_{1}^{*}, s_{2}^{*}, s_{3}^{*}\right)$ is a CPNE of the open multilateral interconnection game $G^{m}$ (respectively, of the bilateral interconnection game $G^{b}$ ) if and only if

(i) $s^{*}$ is a $N E$ of $G^{m}$ (respectively, of $G^{b}$ )

(ii) for any pair of publishers $i, j$, there is no $N E$ of $G_{i, j}^{m}\left(s_{k}^{*}\right)$ (respectively, of $G_{i, j}^{b}\left(s_{k}^{*}\right)$ ) in which the profits of $i$ and $j$ are both strictly higher than $\pi_{i}\left(s^{*}\right)$ and $\pi_{j}\left(s^{*}\right)$, respectively.

In fact, in order to apply the definition of Bernheim, Peleg and Whinston (1987) we should also verify that there is no $s$ which satisfies (i)-(ii) and such that $\pi_{i}(s)>\pi_{i}\left(s^{*}\right)$ for $i=1,2,3$. But this condition is certainly satisfied, since $\pi_{1}(s)+\pi_{2}(s)+\pi_{3}(s)=M$ holds for any s. In short, CPNE eliminates the NE of $G^{m}$ (respectively, of $G^{b}$ ) for which there exists a profitable joint deviation for publishers $i$ and $j$, provided that this joint deviation is a NE in the game between $i$ and $j$, taking as fixed the strategy of publisher $k$. By applying CPNE, we obtain a unique equilibrium outcome both in $G^{m}$ and $G^{b}$, except for non-generic parameter values.

We mention, however, a well-known refinement for the Myerson game, which is called pairwise stable NE. When applied to $G^{b}$, this refinement eliminates a NE $s^{*}$ if $i$ and $j$ are not linked under $s^{*}$, and the creation of the link between $i$ and $j$ increases the profit of at least one of these two players without reducing the profit of the other. Thus, pairwise stable NE are NE which are robust to deviations by any pair of players, when deviations are restricted to generating a link between a pair of deviating players which initially does not exist. ${ }^{32}$ In our context, pairwise stability differs from CPNE because of this restriction on possible joint deviations, and because these are not required to be a NE of $G_{i, j}^{b}\left(s_{k}^{*}\right)$. In

\footnotetext{
${ }^{32}$ For instance, Bloch and Belleflamme (2004) use pairwise stable NE as a solution concept. "Simple" Pairwise stability has been introduced by Jackson and Wolinsky (1996) as a robustness criterion for a
} 
particular, the former feature sometimes makes pairwise stability somewhat weak so that there exist multiple pairwise stable NE in $G^{b}$ (see the remark at the end of section 4.3).

The next table summarizes key notation introduced up to now.

Table 1: Notation

\begin{tabular}{ll}
\hline $\mathrm{B}_{i}$ & bundle of journals of publisher $i$ \\
\hline$U_{i}\left(I_{i j}\right)$ & stand-alone value of $\mathrm{B}_{i}$ (value from interconnection of $\left.\mathrm{B}_{i} \& \mathrm{~B}_{j}\right)$ \\
\hline$A$ & set of active publishers \\
\hline$\Gamma^{m}\left(\Gamma^{b}\right)$ & open multilateral (bilateral) interconnection game \\
\hline$\gamma_{I I}\left(\gamma_{I I I}\right)$ & subgame starting in stage two (stage three) \\
\hline$x_{i}$ & $i$ 's interconnection strategy in multilateral interconnection game \\
\hline $\mathbf{x}_{i}=\left(x_{i j}, x_{i k}\right)$ & $i$ 's interconnection strategy in bilateral interconnection game \\
\hline$x \quad(\mathbf{x})$ & interconnection strategy profile $x=\left(x_{1}, x_{2}, x_{3}\right)\left(\mathbf{x}=\left(\mathbf{x}_{1}, \mathbf{x}_{2}, \mathbf{x}_{3}\right)\right)$ \\
\hline$s_{i}$ & $i$ 's interconnection strategy: either $s_{i}=x_{i}$ or $s_{i}=\mathbf{x}_{i}$ \\
\hline$y$ & interconnection profile $y=\left(y_{12}, y_{13}, y_{23}\right)$ \\
\hline$M^{t}(y)$ & given $y$, the threshold value of budget that allows 3 \\
\hline$P_{i}^{t}(y)$ & to realize a positive profit \\
\hline$P_{i}^{d}$ & given $y, i$ 's profit under triopoly when $M>M^{t}(y)$ \\
\hline$G^{m}\left(G^{b}\right)$ & one-stage game of open multilateral (bilateral) interconnection \\
\hline$G_{i j}^{m}\left(s_{k}\right)$ & $G^{m}$ played between $i$ and $j$ given that $k$ plays $s_{k}$ \\
\hline$G_{i j}^{b}\left(s_{k}\right)$ & $G^{b}$ played between $i$ and $j$ given that $k$ plays $s_{k}$ \\
\hline
\end{tabular}

\subsection{Open multilateral interconnection}

We now examine the one-stage game of open multilateral interconnection $G^{m}$. Since $M^{t}(y)$ is increasing in $y_{12}$ and decreasing in $\left(y_{13}, y_{23}\right)$, we note that the following inequalities hold;

$$
U_{1}-U_{2}<M^{t}(0,1,0)<M^{t}(0,0,1)<M^{t}(0,0,0)<M^{t}(1,1,1)<M^{t}(1,0,0)<U-I_{12}
$$

For $M$ between $U_{1}-U_{2}$ and $M^{t}(0,1,0)$, even the interconnection profile the most favorable for 3 under $G^{m}$ (i.e. $y=(0,1,0)$ ) does not allow 3 to realize a positive profit. Then, there is duopoly and the interconnection profile does not affect the profits of 1 and 2. Hence, we consider $M>M^{t}(0,1,0)$. Let $x=\left(x_{1}, x_{2}, x_{3}\right)$ denote a strategy profile in $G^{m}$. The next lemma shows how CPNE rules out a particular strategy profile:

network, without any reference to the Myerson game or to any other non-cooperative procedure of network formation. Precisely, a network is said to be pairwise stable if (i) no player can gain from severing an existing link he has with another player; (ii) for any pair of players $i, j$ which are not linked, if the creation of the link between $i$ and $j$ increases the profit of $i$, then it reduces the profit of $j$. For instance, Goyal and Moraga-Gonzales (2001) use pairwise stability. 
Lemma 4 The interconnection strategy profile $x=(0,0,0)$ is not a CPNE of the open multilateral interconnection game $G^{m}$ as long as $M>M^{t}(0,1,0)$.

To illustrate how CPNE is applied in the lemma, we consider the case of $M^{t}(0,0,0)<$ $M \leq M^{t}(1,0,0)$. Then $G_{1,2}^{m}(0)$, in which $x_{3}$ is fixed at 0 , is defined as follows:

$$
\begin{array}{cc|c|c|}
\multicolumn{1}{c}{1 \backslash 2} & \multicolumn{1}{c}{x_{2}=0} & \multicolumn{1}{c}{x_{2}=1} \\
\cline { 3 - 4 } G_{1,2}^{m}(0): & x_{1}=0 & P_{1}^{t}(0,0,0), P_{2}^{t}(0,0,0) & P_{1}^{t}(0,0,0), P_{2}^{t}(0,0,0) \\
\cline { 3 - 4 } & x_{1}=1 & P_{1}^{t}(0,0,0), P_{2}^{t}(0,0,0) & P_{1}^{d}, P_{2}^{d} \\
\cline { 3 - 4 } & &
\end{array}
$$

In $G_{1,2}^{m}(0)$, we have two NEs: $\left(x_{1}, x_{2}\right)=(0,0)$ is a NE and, since $P_{1}^{d}>P_{1}^{t}(0,0,0)$ and $P_{2}^{d}>P_{2}^{t}(0,0,0)$, also $\left(x_{1}, x_{2}\right)=(1,1)$ is a NE. Moreover, $\left(x_{1}, x_{2}\right)=(1,1)$ strictly Pareto dominates $\left(x_{1}, x_{2}\right)=(0,0)$ for publishers 1 and 2 . Therefore, condition (ii) in Definition 1 is not satisfied when $x=(0,0,0)$, and this profile is not a CPNE for $M^{t}(0,0,0)<$ $M \leq M^{t}(1,0,0)$. Intuitively, when one publisher does not interconnect, interconnection between the remaining publishers improves their competitive positions and hence they have an incentive to interconnect, which makes $x=(0,0,0)$ not a CPNE.

The notion of CPNE does not deliver uniqueness in a strict sense, but it yields uniqueness in the sense that when multiple CPNE exist, they are all outcome equivalent in terms of active publishers and prices. The next proposition establishes that (i) for $M \leq M^{t}(1,1,1)$, full interconnection $x=(1,1,1)$ is a CPNE and any CPNE generates the same outcome, in terms of active publishers and prices, as full interconnection; (ii) for $M>M^{t}(1,1,1)$, full interconnection is the unique CPNE.

Proposition 2 Consider the open multilateral interconnection game $G^{m}$. Under $A 1^{w}$ :

(i) When $U_{1}-U_{2}<M \leq M^{t}(1,1,1)$, full interconnection is a CPNE of $G^{m}$ and any CPNE is outcome equivalent in terms of active publishers and prices: only publisher 1 and publisher 2 are active and charge $P_{i}^{*}=P_{i}^{d}$ for $i=1,2$;

(ii) When $M^{t}(1,1,1)<M \leq U-I_{12}$, full interconnection is the unique CPNE of $G^{m}$ and hence all three publishers are active and charge $P_{i}^{*}=P_{i}^{t}(1,1,1)$ for $i=1,2,3$.

A main difference with respect to what happens without interconnection (i.e., in $\Gamma^{0}$ ) is that now 3 is active if and only if $M>M^{t}(1,1,1)$. Since $M^{t}(1,1,1)>M^{t}(0,0,0)$, this implies that an open multilateral platform makes the exclusion of the smallest publisher, publisher 3, more likely than in the absence of the platform.

In order to provide an intuition for the result in this proposition, it is useful to consider the case of $M>M^{t}(1,0,0)$, which means that the budget is large enough that even the interconnection profile the least favorable for 3 allows him to sell $\mathrm{B}_{3}$ at a strictly positive price; therefore, all publishers will be active and profits will be $P_{1}^{t}(y), P_{2}^{t}(y), P_{3}^{t}(y)$. Since we have ruled out above the case of $x=(0,0,0)$, we infer that $x_{i}=1$ for at least one 
publisher $i$, and then we see that publisher $j(\neq i)$ prefers to play $x_{j}=1$ rather than $x_{j}=0$. Indeed, $x_{j}=1$ strictly increases $y_{j i}$ and weakly increases $y_{j k}$, which in turn increases the price $P_{j}^{t}(y)$ of $\mathrm{B}_{j}$ because the value of $\mathrm{B}_{i} \& \mathrm{~B}_{j}$ (and perhaps also that of $\left.\mathrm{B}_{j} \& \mathrm{~B}_{k}\right)$ has increased [see (1)-(2) and the related discussion]. In other words, since in our model each publisher competes for relative standing, as the industry profit is equal to $M$ and is constant, $x_{j}=1$ strictly improves the relative standing of $\mathrm{B}_{j}$ compared to $x_{j}=0$, as long as there is an $i$ with $x_{i}=1$, and thereby increases $j$ 's profit. This implies that $x=(1,1,1)$ is the unique CPNE when $M>M^{t}(1,0,0) \cdot{ }^{33}$

Consider now $M \leq M^{t}(1,0,0)$. Then, whether 3 is active or not depends on the interconnection profile and thus publisher 1's (or 2's) incentive to interconnect may be affected by the possibility to exclude 3 . Obviously, 3 chooses $x_{3}=1$ since his interconnection increases the chance to be active by lowering the threshold $M^{t}$. Furthermore, the possibility to exclude 3 does not affect 1's or 2's incentive to interconnect. For instance, if 2 interconnects, 1 wants to interconnect in order to avoid the interconnection profile $y_{12}=y_{13}=0, y_{23}=1$, which is the least favorable for 1 . If 2 does not interconnect, it is still optimal for 1 to interconnect (so that $y_{13}=1, y_{12}=y_{23}=0$ ). Therefore, it is easier for 3 to be active when 2 does not interconnect than when 2 interconnects.

Given that the interconnection technology exhibits economies of scale, full interconnection of all publishers worsens the relative standing of the small publisher, which explains why 3 is less often active under the open multilateral interconnection than in the absence of interconnection.

\subsection{Bilateral interconnections}

We now consider the one-stage game of bilateral interconnection $G^{b}$. We find that several cases may arise depending on the relative values of $I_{12}, I_{13}, I_{23}$. In particular, publisher 3 can be excluded more often or less often in $G^{b}$ than in $G^{m}$. We notice that when all three publishers are active in $G^{b}$, it is possible that they are not fully interconnected, while we have seen that in $G^{m}$ full interconnection is the only CPNE when all publishers are active. We explain below why these results arise in $G^{b}$.

By applying CPNE in $G^{b}$ we obtain a unique equilibrium outcome for any generic parameter values, and in particular the next lemma shows that there are three possible CPNE outcomes: either 3 is excluded, or 3 is active and $y=(0,1,1)$, or 3 is active and $y=(1,1,1)$.

Lemma 5 If $\mathbf{x}^{*}$ is a CPNE of the bilateral interconnection game $G^{b}$ such that 3 is active, then $\mathbf{x}^{*}$ generates as interconnection profile either $y=(0,1,1)$ or $y=(1,1,1)$.

\footnotetext{
${ }^{33}$ This intuition holds regardless of whether the mode of interconnection is multilateral or bilateral.
} 
Lemma 5 essentially says that $y_{13}=y_{23}=1$ in any CPNE in which 3 is active. Therefore, when we investigate the existence of a CPNE in which 3 is active, the only indeterminacy is about whether $y_{12}=0$ or $y_{12}=1$. The intuition for this result is that if a strategy profile $\mathbf{x}$ is such that 3 is active and $y_{13}=0$, then in $G_{1,3}^{b}\left(\mathbf{x}_{2}\right)$ there exists a NE in which 1 and 3 are interconnected and they are both better off than in $\mathbf{x}$, which implies that $\mathbf{x}$ is not a CPNE; the same argument applies if $y_{23}=0$, with reference to $G_{2,3}^{b}\left(\mathbf{x}_{1}\right)$. Notice that $y=(0,1,1)$ is a star network in which the center of the star is the smallest publisher.

Because of the greater interconnection flexibility in $G^{b}$ with respect to $G^{m}$, the values of $M$ in the interval $\left[U_{1}-U_{2}, U-I_{12}\right]$ need to be divided more finely than in $G^{m}$, and it is useful to recall that the following inequalities hold

$$
\begin{aligned}
U_{1}-U_{2} & <M^{t}(0,1,1)<M^{t}(0,1,0)<M^{t}(0,0,1)<M^{t}(0,0,0) \\
& <M^{t}(1,1,1)<M^{t}(1,1,0)<M^{t}(1,0,1)<M^{t}(1,0,0)<U-I_{12}
\end{aligned}
$$

From the arguments in sections 4.1-4.2, the following results are straightforward. For $U_{1}-U_{2}<M \leq M^{t}(0,1,1), y$ does not matter and only 1 and 2 are active since even the interconnection profile the most favorable for $3, y=(0,1,1)$, does not allow him to make a profit. Similarly, for $M^{t}(0,1,1)<M \leq M^{t}(0,1,0)$ only 1 and 2 are active: 2 has an incentive to choose $x_{23}=0$ in order to exclude 3 since $P_{2}^{d}>P_{2}^{t}(0,1,1)$. For $M^{t}(1,0,0)<M \leq U-I_{12}$, all publishers are active and fully interconnected since even the interconnection profile the least favorable for $3, y=(1,0,0)$, allows him to make a profit. For expositional simplicity, Proposition 3 presents a weak version of our results restricting attention to the case in which $2 I_{12}>I_{13}+3 I_{23}$ for $M$ between $M^{t}(1,1,1)$ and $M^{t}(1,0,1)$, while in the proof in the appendix we present (and prove) a more general version of Proposition 3 without the parameter restriction. In particular, $2 I_{12}>I_{13}+3 I_{23}$ implies $P_{2}^{d} \geq P_{2}^{t}(0,0,1)$ when $M>M^{t}(1,1,1)$ and hence makes 2 strongly prefer exclusion of $3 .^{34}$

Proposition 3 Under A1, any CPNE of the bilateral interconnection game $G^{b}$ is such that

(i) for $M$ between $U_{1}-U_{2}$ and $M^{t}(0,1,0)$ : $A^{*}=\{1,2\}$.

(ii) for $M$ between $M^{t}(0,1,0)$ and $M^{t}(1,1,1)$ :

(a) if $P_{1}^{d}>P_{1}^{t}(0,1,1)$, then $A^{*}=\{1,2\}$. In this case, $\mathbf{x}_{1}^{*}=\mathbf{x}_{2}^{*}=(1,1), \mathbf{x}_{3}^{*}=(0,0)$ is a CPNE.

(b) if $P_{1}^{d}<P_{1}^{t}(0,1,1)$, then $A^{*}=\{1,2,3\}$ with $y=(0,1,1)$. In this case, $\mathbf{x}_{1}^{*}=\mathbf{x}_{2}^{*}=$ $(0,1), \mathbf{x}_{3}^{*}=(1,1)$ is a CPNE.

(iii) for $M$ between $M^{t}(1,1,1)$ and $M^{t}(1,1,0)$ and under $2 I_{12}>I_{13}+3 I_{23}$ :

\footnotetext{
${ }^{34}$ In addition, it implies $P_{1}^{t}(1,1,1)>P_{1}^{t}(0,1,0)$.
} 
(a) if $P_{1}^{d}>P_{1}^{t}(0,1,0)$, then $A^{*}=\{1,2\}$. In this case, $\mathbf{x}_{1}^{*}=(1,1), \mathbf{x}_{2}^{*}=(1,0)$, $\mathbf{x}_{3}^{*}=(0,0)$ is a CPNE.

(b) if $P_{1}^{d}<P_{1}^{t}(0,1,0)$, then $A^{*}=\{1,2,3\}$ with $y=(0,1,1)$. In this case, $\mathbf{x}_{1}^{*}=\mathbf{x}_{2}^{*}=$ $(0,1), \mathbf{x}_{3}^{*}=(1,1)$ is a CPNE.

(iv) for $M$ between $M^{t}(1,1,0)$ and $M^{t}(1,0,1)$ and under $2 I_{12}>I_{13}+3 I_{23}$ :

(a) if $P_{1}^{d}>P_{1}^{t}(1,1,1)$, then $A^{*}=\{1,2\}$. In this case, $\mathbf{x}_{1}^{*}=(1,0), \mathbf{x}_{2}^{*}=(1,1)$, $\mathbf{x}_{3}^{*}=(0,0)$ is a CPNE.

(b) if $P_{1}^{d}<P_{1}^{t}(1,1,1)$, then $A^{*}=\{1,2,3\}$ with $y=(1,1,1)$. In this case, full interconnection $\mathbf{x}_{1}^{*}=\mathbf{x}_{2}^{*}=\mathbf{x}_{3}^{*}=(1,1)$ is the unique CPNE.

(v) for $M$ between $M^{t}(1,0,1)$ and $U-I_{12}: A^{*}=\{1,2,3\}$ with $y=(1,1,1)$. In this case, full interconnection $\mathbf{x}_{1}^{*}=\mathbf{x}_{2}^{*}=\mathbf{x}_{3}^{*}=(1,1)$ is the unique CPNE.

This proposition reveals that the outcome of $G^{b}$ is the same as that of $G^{m}$ if $M \leq$ $M^{t}(0,1,0)$ or if $M>M^{t}(1,0,1)$ but is often quite different otherwise. For $M$ between $M^{t}(0,1,0)$ and $M^{t}(1,1,0)$, we have either duopoly or triopoly (with 3 as the center of a star) while for $M$ between $M^{t}(1,1,0)$ and $M^{t}(1,0,1)$, we have either duopoly or triopoly (with full interconnection). As we explain below, the outcome between duopoly and triopoly is determined by the existence of a (self-enforcing) ${ }^{35}$ joint deviation which is profitable for 1 and for another publisher (as required by CPNE). Except for the case of $M$ between $M^{t}(1,1,1)$ and $M^{t}(1,1,0)$, the existence of such a deviation is determined by 1 's trade-off between the profit upon excluding 3 and the profit upon inducing 3 to be active, which takes the form of $P_{1}^{d} \gtrless P_{1}^{t}(y)$, where the interconnection profile $y$ in $P_{1}^{t}(y)$ varies depending on the level of $M$.

We consider first $M$ between $M^{t}(0,1,0)$ and $M^{t}(1,1,1)$ and explain why $P_{1}^{t}(0,1,1)>$ $P_{1}^{d}$ leads to $A^{*}=\{1,2,3\}$ and $y=(0,1,1)$. We start by noticing that there exists a NE $\mathbf{x}^{\prime}$ of $G^{b}$, such as $\mathbf{x}_{1}^{\prime}=\mathbf{x}_{2}^{\prime}=(1,1), \mathbf{x}_{3}^{\prime}=(0,0)$, which leads to exclusion of 3. However, when $P_{1}^{t}(0,1,1)>P_{1}^{d}$ we can find a NE in $G_{1,3}^{b}\left(\mathbf{x}_{2}^{\prime}\right)$ which gives both 1 and 3 higher profits than when 3 is excluded, namely $\mathbf{x}_{1}=(0,1), \mathbf{x}_{3}=(1,1)$ (which induces $y=(0,1,1)$ and makes 3 active). Therefore, if $P_{1}^{t}(0,1,1)>P_{1}^{d}, \mathbf{x}^{\prime}$ is not a CPNE and, for the same reason, 3 must be active in any CPNE. Furthermore, since $M<M^{t}(1,1,1)$, if 3 is active then $y=(0,1,1)$ from Lemma 5 ; actually, we find that $\mathbf{x}^{*}$ with $\mathbf{x}_{1}^{*}=\mathbf{x}_{2}^{*}=(0,1), \mathbf{x}_{3}^{*}=(1,1)$ is a CPNE. Notice that 2's duopoly profit $P_{2}^{d}$ is larger than $P_{2}^{t}(0,1,1)$, thus 2 always gains from deviating jointly with 1 (by playing $\mathbf{x}_{1}=\mathbf{x}_{2}=(1,1)$ ) in order to exclude 3. Nevertheless, if $P_{1}^{t}(0,1,1)>P_{1}^{d}$, such deviation is not profitable for 1 . If instead $P_{1}^{d}>P_{1}^{t}(0,1,1), 1$ prefers duopoly to $y=(0,1,1)$ and 3 becomes excluded.

Since $P_{1}^{t}(0,1,1)=U_{1}+\frac{1}{3}\left(M-U+I_{13}-2 I_{23}\right)$ and $P_{1}^{d}$ does not depend on $I_{i j}$, $P_{1}^{t}(0,1,1)>P_{1}^{d}$ holds if $I_{13}$ is large enough with respect to $I_{23}$, which means that pub-

\footnotetext{
${ }^{35}$ Self-enforcing in the sense that the joint deviation is a NE.
} 
lisher 1 is much larger than 2. Basically, in this case, 1 breaks connectivity with 2 while maintaining it with 3 for differentiation motive (i.e. to further differentiate himself from 2 ); since 1's gain from interconnection with 3 is much larger than 2's gain from interconnection with 3 , this improves 1's relative standing and makes 1's profit larger than his profit from excluding 3 . If instead 1 is not much larger than 2 , then $I_{13} \simeq I_{23}$ and 1 maintains connectivity with 2 for exclusion motive (i.e. to exclude 3 ).

Consider now $M$ between $M^{t}(1,1,1)$ and $M^{t}(1,1,0)$ and $2 I_{12}>I_{13}+3 I_{23}$. In this case we find that full interconnection is never a NE; since $P_{2}^{d}>P_{2}^{t}(1,1,1), 2$ has an incentive to exclude 3 by choosing $x_{23}=0$ (this result does not depend on $2 I_{12}>I_{13}+3 I_{23}$ ). Hence, Lemma 5 implies that either 3 is excluded, or he is active with $y=(0,1,1)$. The latter is a CPNE outcome if $P_{1}^{t}(0,1,0)>P_{1}^{d}$. In order to explain the inequality, we note that $\mathbf{x}^{*}$ such that $\mathbf{x}_{1}^{*}=\mathbf{x}_{2}^{*}=(0,1), \mathbf{x}_{3}^{*}=(1,1)$ is a NE of $G^{b}$. But if $P_{1}^{d}>P_{1}^{t}(0,1,1)$, there are Pareto improving joint deviations of 1 and 2 in $G_{1,2}^{b}\left(\mathbf{x}_{3}^{*}\right)$ which exclude 3 . Nevertheless, if $P_{1}^{t}(0,1,0)>P_{1}^{d}$ then none of these deviations is a NE, which makes $\mathbf{x}^{*}$ a CPNE. ${ }^{36}$

Consider finally $M$ between $M^{t}(1,1,0)$ and $M^{t}(1,0,1)$ and $2 I_{12}>I_{13}+3 I_{23}$. Suppose $P_{1}^{d}>P_{1}^{t}(1,1,1)$. Then, $y=(1,1,1)$ cannot occur in equilibrium because if $\mathbf{x}_{2}=\mathbf{x}_{3}=$ $(1,1)$, then 1 prefers to exclude 3 by playing $\mathbf{x}_{1}=(1,0)$. The profile $y=(0,1,1)$ cannot arise either because in $G_{1,2}^{b}(1,1)$ there is a NE (such as $\mathbf{x}_{1}=(1,0), \mathbf{x}_{2}=(1,1)$ ) which excludes 3 and yields a Pareto improvement to 1 and 2. Therefore, Lemma 5 implies that 3 is not active. In the opposite case of $P_{1}^{d}<P_{1}^{t}(1,1,1)$, publisher 3 is active in any CPNE because, if he is not active, there exists a profitable joint deviation for 1 and 3 [here $P_{1}^{d}<P_{1}^{t}(1,1,1)$ matters] which is a NE in $G_{1,3}^{b}\left(\mathbf{x}_{2}\right)$. Furthermore, $y=(1,1,1)$ because, if $y=(0,1,1)$, then it is a NE in $G_{1,2}^{b}\left(\mathbf{x}_{3}\right)$ to create the link between 1 and 2 .

When we compare $G^{m}$ and $G^{b}$, we find three interesting differences. First, there is less interconnection in $G^{b}$ than in $G^{m}$. Recall that in $G^{m}$ full interconnection is an outcome of CPNE and is the unique outcome for $M>M^{t}(1,1,1)$. Surprisingly, in $G^{b}$, the smallest publisher can emerge as the center of a star network. Second, partial interconnection in $G^{b}$ leads to less exclusion than in $G^{m}$ if it results from 1's differentiation motive (i.e. when 3 is the center of a star); otherwise, it leads to more exclusion. Last, exclusion of 3 is less likely in $G^{m}$ as $M$ increases. However, this monotonicity does not hold in $G^{b}$. For instance, when $M$ is between $M^{t}(0,1,0)$ and $M^{t}(1,1,1)$, we could have $P_{1}^{d}<P_{1}^{t}(0,1,1)$ so that 3 is active - for $M$ slightly larger than $M^{t}(0,1,0)$, but $P_{1}^{d}>P_{1}^{t}(0,1,1)$ - so that 3 is excluded - for $M$ close to $M^{t}(1,1,1)$. This is because, as $M$ increases, 1 's duopoly profit increases faster than 1's triopoly profit since the former increases by a factor of $1 / 2$ while the latter by a factor of $1 / 3$.

Remark 1 (pairwise stable NEs): For $M$ between $M^{t}(0,0,0)$ and $M^{t}(1,1,1)$, if

\footnotetext{
${ }^{36}$ When $M<M^{t}(1,1,1)$, instead, $P_{1}^{d}>P_{1}^{t}(0,1,1)$ suffices to destroy $\mathbf{x}^{*}$ because $\mathbf{x}_{1}=\mathbf{x}_{2}=(1,1)$ induces 3 to be inactive and is a NE of $G_{1,2}^{b}\left(\mathbf{x}_{3}^{*}\right)$.
} 
$P_{1}^{t}(0,1,1)>P_{1}^{d}$ then there exist two pairwise stable NEs: $\mathbf{x}_{1}^{*}=\mathbf{x}_{2}^{*}=(1,0), \mathbf{x}_{3}^{*}=(0,0)$ and also $\mathbf{x}_{1}^{*}=\mathbf{x}_{2}^{*}=(0,1), \mathbf{x}_{3}^{*}=(1,1)$.

\section{Endogenous interconnection regime}

In this section, we address the issue of market provision of an open multilateral interconnection platform by endogenizing the creation of the platform. Actually, OpenSocial was created by Google, and CrossRef was created by the initiative of big commercial publishers. In the context of our model, we ask when the largest publisher has an incentive to create CrossRef. Addressing this question requires us to study what we call a hybrid interconnection game in which publishers can interconnect both bilaterally and through an open multilateral platform. This is because, even though an open multilateral platform is created, publishers would still have the freedom to sign bilateral agreements to interconnect.

In the next subsection we study the hybrid interconnection game, in which we assume that the open multilateral interconnection platform already exists. This analysis allows to understand publisher 1's incentive to create such a platform.

\subsection{Hybrid interconnection game}

In this section, we study a one-stage game of hybrid interconnection, denoted by $G^{h}$, which is defined as follows. The interconnection strategy space for each publisher is $\{0,1\}^{3}$, and a strategy for publisher $i$ is $X_{i}=\left(x_{i}, x_{i j}, x_{i k}\right)$, where $x_{i}$ is $i$ 's action to interconnect through the multilateral platform and $x_{i j}\left(x_{i k}\right)$ represents $i$ 's action to bilaterally interconnect with $j$ (with $k$ ). Publishers $i$ and $j$ are interconnected, that is $y_{i j}=1$, if $x_{i}=x_{j}=1$ and/or $x_{i j}=x_{j i}=1$, otherwise $y_{i j}=0$. Thus, $G^{h}$ is defined by taking $s_{i}=X_{i}$ in section 4.1: a given strategy profile $s=\left(s_{1}, s_{2}, s_{3}\right)$ determines an interconnection profile $y(s)$, which in turn determines each publisher's profit from Lemma 3 as in (4).

The next proposition describes CPNEs of $G^{h}$ :

Proposition 4 Consider the hybrid interconnection game $G^{h}$ and assume A1.

(i) Any CPNE outcome of the bilateral interconnection game is a CPNE outcome of the hybrid interconnection game in the following sense: if $\mathbf{x}^{*}=\left(\mathbf{x}_{1}^{*}, \mathbf{x}_{2}^{*}, \mathbf{x}_{3}^{*}\right)$ is a CPNE of the bilateral interconnection game, then the profile of strategies $X_{1}^{*}=\left(0, \mathbf{x}_{1}^{*}\right), X_{2}^{*}=\left(0, \mathbf{x}_{2}^{*}\right)$, $X_{3}^{*}=\left(0, \mathbf{x}_{3}^{*}\right)$ is a CPNE of the hybrid interconnection game.

(ii) Assume $2 I_{12}>I_{13}+3 I_{23}$. Then there exists a CPNE outcome of the hybrid interconnection game which is different from a CPNE outcome of the bilateral interconnection game only if $M^{t}(1,1,1)<M \leq M^{t}(1,1,0)$ and $P_{1}^{t}(1,1,1)>P_{1}^{d}$. In this case, the full 
interconnection outcome (that is, the CPNE outcome of the open multilateral interconnection game) is a CPNE outcome of the hybrid interconnection game, delivered by the strategy profile $\hat{X}_{1}=\hat{X}_{2}=\hat{X}_{3}=(1,0,0)$.

This proposition says that for some parameters, in $G^{h}$ there are multiple CPNE outcomes. Precisely, any CPNE outcome of the bilateral interconnection game is a CPNE outcome of the hybrid interconnection game and, in addition, when $P_{1}^{t}(1,1,1)>P_{1}^{d}$ and $M$ is between $M^{t}(1,1,1)$ and $M^{t}(1,1,0)$, we find that the full interconnection outcome of the open multilateral interconnection game is also a CPNE outcome of the hybrid interconnection game.

\subsection{Market provision of open multilateral platform}

Now we suppose that publisher 1 has the possibility to create CrossRef by paying a certain $\operatorname{cost} c>0$, and thus we consider a game in which first he decides whether to create or not CrossRef, and then the three publishers play the hybrid interconnection game $G^{h}$ (in the first case) or the bilateral interconnection game $G^{b}$ (in the second case). Proposition 4 reveals that the creation of CrossRef may have an effect only when $M$ is between $M^{t}(1,1,1)$ and $M^{t}(1,1,0)$ and $P_{1}^{t}(1,1,1)>P_{1}^{d: 37}$ in such a case full interconnection is a CPNE outcome, in addition to the CPNE outcome of $G^{b}$ (which depends on the parameters). Therefore, there is a multiplicity problem if $G^{h}$ is entered, but it is important to notice that 1's profit under full interconnection is $P_{1}^{t}(1,1,1)$, which is higher than 1 's profit in $G^{b}$ as $P_{1}^{t}(1,1,1)>P_{1}^{t}(0,1,1)$ and $P_{1}^{t}(1,1,1)>P_{1}^{d}$. Then, for $c>0$, (essentially) the same forward induction argument invoked in Cabrales and Calvò-Armengol (2007) suggests that if 1 decides to create CrossRef, then he will not play in $G^{h}$ the interconnection action which is consistent with the CPNE of $G^{b}$, as that is a weakly dominated strategy for him. In other words, creating CrossRef can be interpreted as a credible signal which reveals 1's intention to play the full interconnection equilibrium in $G^{h}$. In the next corollary, we assume that publisher 1 avoids weakly dominated strategies in the game of creation of an open multilateral interconnection platform.

Corollary 1 (i) Suppose that $M^{t}(1,1,1)<M \leq M^{t}(1,1,0)$ and $c<\max \left\{P_{1}^{t}(1,1,1)-\right.$ $\left.P_{1}^{d}, P_{1}^{t}(1,1,1)-P_{1}^{t}(0,1,1)\right\}$. Then, the largest publisher creates an open multilateral platform and the full interconnection outcome is realized.

(ii) This creation of the open multilateral interconnection platform by the largest publisher is welfare improving.

\footnotetext{
${ }^{37}$ In any other case, $G^{b}$ and $G^{h}$ have the same CPNE outcome.
} 
In particular, we notice that the market provision of the open multilateral interconnection platform is welfare improving because it either induces 3 to be active or improves interconnection between 1 and 2 .

\section{For-profit platform for multilateral interconnection}

In this section we consider a monopoly for-profit platform for multilateral interconnection. ${ }^{38}$ The platform charges fees on each publisher who wants to interconnect, in order to maximize her profit, that is her revenue. Let $f_{i}>0$ denote the fee which publisher $i$ is required to pay in order to interconnect, for $i=1,2,3$. Formally, we consider a game in which first the for-profit platform chooses $f_{1}, f_{2}, f_{3}$, and then publishers, after observing the fees, play the multilateral interconnection game $G^{m}$ augmented with $f_{1}, f_{2}, f_{3}$. This means that given $x=\left(x_{1}, x_{2}, x_{3}\right)$, the profit of publisher $i$ is $\pi_{i}(x)$ if $x_{i}=0$ and is $\pi_{i}(x)-f_{i}$ if $x_{i}=1$, with $\pi_{i}(x)$ defined in (4). For simplicity, we continue to use $G^{m}$ to represent the multilateral interconnection game with given $f_{1}, f_{2}, f_{3}$, but we let $G^{f m}$ represent the multilateral interconnection game that starts with the for-profit platform's choice of fees.

As before, we apply CPNE to find equilibrium outcome(s) of $G^{m}$ for given $f_{1}, f_{2}, f_{3}$. This makes it quite challenging to determine the revenue maximizing fees. We solve this problem by using a two-step procedure in the spirit of mechanism design. As a first step, for any strategy profile $x$ we determine the revenue maximizing fees among the fees which induce publishers to play $x$, denoted by $f_{1}(x), f_{2}(x), f_{3}(x)$; this is analogous to finding the highest revenue for a principal, given the allocation he wants to implement. As a second step, given the results from the previous step, we select the $x$ with the highest associated revenue, that is the $x$ which maximizes $x_{1} f_{1}(x)+x_{2} f_{2}(x)+x_{3} f_{3}(x)$; this is similar to determining the allocation which maximizes the revenue, given the result of the previous step.

It is important to notice that the only interesting interconnection strategy profiles for the for-profit platform are $x=(1,1,1), x=(1,1,0), x=(1,0,1), x=(0,1,1)$, since the platform cannot make any money from inducing the publishers to play $x=(0,0,0)$, and it is impossible to induce the publishers to play $x=(0,1,0)$, or $x=(0,0,1)$, or $x=(1,0,0)$. Indeed, $x=(1,0,0)$, for instance, determines the interconnection profile $y=(0,0,0)$, exactly like $x=(0,0,0)$, and thus it is not a best reply for 1 to play $x_{1}=1$ as it has no effect on $y$ but costs $f_{1}>0$ to 1 .

As we have noticed above, if $M \leq M^{t}(0,1,0)$ then 3 cannot be active, independently of the interconnection profile. Then necessarily $x_{1}=x_{2}=0$, and thus the platform cannot

\footnotetext{
${ }^{38}$ For bilateral interconnections, it is much less interesting to consider a for-profit platform since any pair of publishers can sign bilateral agreements without going through the platform.
} 
make money when $M \leq M^{t}(0,1,0)$. Therefore, we only consider the values of $M$ between $M^{t}(0,1,0)$ and $U-I_{12}$.

Proposition 5 Consider a monopoly for-profit platform for multilateral interconnection. Under A1;

(i) When $M^{t}(0,1,0)<M \leq M^{t}(0,0,0)$, the revenue maximizing fees are given as follows

$$
f_{1}=P_{1}^{t}(0,1,0)-P_{1}^{d}, \quad f_{2} \text { large }, \quad f_{3}=P_{3}^{t}(0,1,0) .
$$

Then, only publisher 1 and publisher 3 join the platform and all three publishers are active. (ii) When $M^{t}(0,0,0)<M \leq M^{t}(1,1,1)$, the revenue maximizing fees are given as follows.

$$
\begin{array}{r}
f_{1}=P_{1}^{t}(0,1,0)-P_{1}^{d}, \quad f_{2} \text { large, } \quad f_{3}=P_{3}^{t}(0,1,0) \quad \text { if } \frac{2}{3} I_{13}>P_{3}^{t}(0,0,0) \\
f_{1}=f_{2}=P_{1}^{d}-P_{1}^{t}(0,0,0), \quad f_{3} \text { large } \quad \text { if } \frac{2}{3} I_{13}<P_{3}^{t}(0,0,0)
\end{array}
$$

In the first case, only publisher 1 and publisher 3 join the platform and all three publishers are active. In the second case, only publisher 1 and publisher 2 join the platform and publisher 3 is not active.

(iii) When $M^{t}(1,1,1)<M \leq U-I_{12}$, the optimal fees are complicated functions of the parameters, ${ }^{39}$ but in any case it is optimal for the platform to select fees such that all publishers join the platform and are active.

The most interesting result in the above proposition is that exclusion of 3 occurs in $G^{f m}$ only for $M$ between $M^{t}(0,0,0)$ and $M^{t}(1,1,1)$, and if $I_{13}$ is not too large. Therefore, 3 is excluded less often when the multilateral interconnection is mediated by a for-profit platform (i.e. in $G^{f m}$ ) than when it is mediated by an open platform (i.e. in $G^{m}$ ), since in $G^{m} 3$ is inactive for any $M \leq M^{t}(1,1,1)$. In order to see what generates this result, we consider the case of $M \leq M^{t}(0,0,0)$ [part (i) of the Proposition]. In order to make a profit, the for-profit platform cannot induce publishers to play $x=(1,1,1)$ : 3 will not pay any fee since he is excluded regardless of the value of $x_{3}$. The platform cannot induce $x=(1,1,0)$ either since publisher 1 realizes the same duopoly profit regardless of whether $x_{1}=1$ or $x_{1}=0$. However, the platform can make money by inducing $x=(1,0,1)$ because this allows 3 to be active, and can extract 1's and 3's surplus from interconnection: $P_{1}^{t}(0,1,0)-P_{1}^{d}$ and $f_{3}=P_{3}^{t}(0,1,0)$ respectively. Given that the total (gross) profit of the publishers is equal to $M$, this revenue of the for-profit platform is equal to the reduction in 2's profit resulting from the interconnection between 1 and 3 . Now, if $f_{2}$ were small, then 2 would play $x_{2}=1$, which would induce 3 to be inactive and in turn would make it impossible to earn interconnection fees from 3 ; for this reason the

\footnotetext{
${ }^{39}$ The optimal fees are reported in the appendix of the working paper version, Jeon-Menicucci (2009).
} 
fee for 2 must be large enough. ${ }^{40}$ Intuitively, 3 is more often active in $G^{f m}$ than in $G^{m}$ since for low values of $M$, inducing an interconnection profile which makes 3 active is the only way for the platform to earn a revenue.

Things are quite different for $M$ between $M^{t}(0,0,0)$ and $M^{t}(1,1,1)$. Indeed, in this case 1 and 2 cannot anymore exclude 3 without being interconnected, and thus they are willing to pay for interconnection, $P_{1}^{d}-P_{1}^{t}(0,0,0)$ and $P_{2}^{d}-P_{2}^{t}(0,0,0)$ respectively; these two terms (which turn out to be equal) add up to $P_{3}^{t}(0,0,0)$. Therefore, the platform needs to compare the profit it can make by inducing $x=(1,1,0)$ (which makes 3 inactive) with the profit it can make with $x=(1,0,1)$; this explains the comparison between $\frac{2}{3} I_{13}$ and $P_{3}^{t}(0,0,0)$ in Proposition 5(ii).

Finally, for $M>M^{t}(1,1,1)$, the platform finds full interconnection, that is $x=$ $(1,1,1)$, the most profitable so that all firms are active and interconnected. To give an intuition, we note that what the platform charges as an interconnection fee is roughly ${ }^{41}$ the difference between a publisher's profit with interconnection and his profit without interconnection. As the number of rival publishers who join the platform increases, this difference increases. This argument suggests that the platform's profit is maximized with full interconnection as long as full interconnection leads to no exclusion.

\section{Multilateral interconnection and heterogenous li- braries}

In this section we perform a robustness exercise by showing that under multilateral interconnection through an open platform, all publishers interconnect for any number of heterogenous libraries.

Precisely, we suppose that there are $n(\geq 2)$ number of heterogenous libraries: libraries differ in terms of the budget and the values that they obtain from the bundles offered by the publishers. We use $i^{h}(i=1,2,3)$ to denote the publisher with the $i$ th bundle in terms of the stand-alone value that library $h$ obtains, implying $U_{1^{h}}^{h}>U_{2^{h}}^{h}>U_{3^{h}}^{h}$. This also implies $I_{1^{h} 2^{h}}^{h}>I_{1^{h} 3^{h}}^{h}>I_{2^{h} 3^{h}}^{h}$ where $I_{i j}^{h}$ represents the value that library $h$ obtains from interconnection between $i$ and $j$. Let $U^{h} \equiv U_{1^{h}}^{h}+U_{2^{h}}^{h}+U_{3^{h}}^{h}$. Let $M^{h}(>0)$ represent library $h$ 's budget such that $M^{h} \leq U^{h}-I_{1^{h} 2^{h}}^{h}$. We suppose that A1 ${ }^{w}$ holds for each library - we call $\mathrm{A} 1^{w}$ ' the joint assumption for all libraries:

\footnotetext{
${ }^{40}$ When $M$ is between $M(0,0,1)$ and $M(0,0,0)$, it is also possible for the platform to induce $x=(0,1,1)$, but it turns out that the profit it can make in this way is smaller than the profit with $x=(1,0,1)$ : see step 2 in the proof of Proposition 5 in the appendix of the working paper version, Jeon-Menicucci (2009).

${ }^{41}$ The qualification "roughly" is there since the platform cannot fully extract this difference because some coalitional deviations are allowed by the notion of CPNE.
} 
$\mathrm{A} 1^{w}$ ': For $h=1, \ldots, n, U_{3^{h}}^{h} \geq I_{1^{h} 2^{h}}^{h}$ and $U_{2^{h}}^{h}+I_{2^{h} 3^{h}}^{h} \geq U_{3^{h}}^{h}+I_{1^{h} 3^{h}}^{h}$.

Under these assumptions, we consider a game with the following structure.

- (interconnection) In stage one, each publisher $i$ (for $i=1,2,3$ ) simultaneously decides whether or not to interconnect through CrossRef; we let $x_{i}=1$ if he has joined CrossRef, $x_{i}=0$ otherwise.

- (active/non-active) In stage two, after the moves at stage one have been observed by all publishers and all libraries, each publisher simultaneously decides in which markets he wants to be active; we use $A^{h} \subseteq\{1,2,3\}$ to denote the set of active publishers in the market for library $h$.

- (pricing) In stage three, after the moves at stage two have been observed by all publishers and all libraries, publisher $i$ simultaneously chooses a price $P_{i}^{h} \geq 0$ for $\mathrm{B}_{i}$, for $i \in A^{h}$, for $h=1, \ldots, n$.

- In stage four, each library $h$ chooses bundles to buy from the set $\left\{\mathrm{B}_{i}: i \in A^{h}\right\}$.

Note that the publishers' interconnection decisions affect all libraries in the same way. As before, we adopt the tie-breaking rule that if publisher $i$ expects to make zero profit in the market for library $h$, then in stage two he chooses to be non-active in that market. Each publisher wants to maximize the sum of the profits he earns in the different markets.

We use $\hat{G}^{m}$ to denote the game of multilateral interconnection with heterogenous libraries, and in the next corollary we analyze this game. For this purpose we need to define $P_{i, j}^{d, h}=\frac{1}{2}\left(M^{h}+U_{i}^{h}-U_{j}^{h}\right)$ and $P_{i}^{t, h}(1,1,1)=U_{i}^{h}+\frac{1}{3}\left(M^{h}-U^{h}+I_{i j}^{h}+I_{i k}^{h}-2 I_{j k}^{h}\right)$; $P_{i, j}^{d, h}$ represents the profit of publisher $i$ in market $h$ if $A^{h}=\{i, j\}$, and $P_{i}^{t, h}(1,1,1)$ is the profit of publisher $i$ in market $h$ if all publishers are active, fully interconnected and $M^{h}>M^{t, h}(1,1,1)=U^{h}-3 U_{3^{h}}^{h}+2 I_{1^{h} 2^{h}}^{h}-I_{1^{h} 3^{h}}^{h}-I_{2^{h} 3^{h}}^{h}$.

Corollary 2 Consider the multilateral interconnection though an open platform. Suppose there are $n(\geq 2)$ number of libraries which can be heterogenous in terms of budgets and preferences, but that $A 1^{w}$ ' holds. Then

(i) if $M^{t, h^{\prime}}(1,1,1)<M^{h^{\prime}}$ for at least one library $h^{\prime}$, then the unique CPNE is such that all publishers are interconnected. As a consequence, in the market for library h (for any $h=1, \ldots, n)$ the active publishers and prices are determined as follows:

$$
\begin{gathered}
\text { if } M^{h} \leq U_{1^{h}}^{h}-U_{2^{h}}^{h} \text {, then } A^{* h}=\left\{1^{h}\right\} \text { and } P_{1^{h}}^{* h}=M^{h} \\
\text { if } U_{1^{h}}^{h}-U_{2^{h}}^{h}<M^{h} \leq M^{t, h}(1,1,1) \text {, then } A^{* h}=\left\{1^{h}, 2^{h}\right\} \text { and } P_{1^{h}}^{* h}=P_{1^{h}, 2^{h}}^{d, h}, P_{2^{h}}^{* h}=P_{2^{h}, 1^{h}}^{d, h}
\end{gathered}
$$




$$
\text { if } M^{t, h}(1,1,1)<M^{h} \text {, then } A^{* h}=\{1,2,3\} \text { and } P_{i}^{* h}=P_{i}^{t, h}(1,1,1) \text { for } i=1,2,3 \text {. }
$$

(ii) If $M^{h} \leq M^{t, h}(1,1,1)$ for all libraries $h=1, \ldots, n$, then full interconnection is a CPNE and all CPNEs yield the same outcome in terms of active publishers and prices in each market $h$ as in (5) and (6).

This corollary says that in the setting of multilateral interconnection it is not restrictive to limit the analysis to the case of a single library, as the game with many heterogenous libraries replicates the outcomes of the various single-library games. The reason for this result is that in the game with multiple libraries, the markets for different libraries are linked one to another only by the publishers' interconnection decisions and we know from Proposition 2 that all publishers have an incentive to interconnect when they consider the market for a single library $h$, an incentive which is strict if $M^{h}>M^{t, h}(1,1,1)$. Since choosing $x_{i}=1$ never hurts publisher $i$ in any market (independently of how the library values $\mathrm{B}_{i}$ ), we obtain that each publisher will interconnect if $M^{h}>M^{t, h}(1,1,1)$ for at least one library.

\section{Symmetric publishers}

In the above sections we have often assumed that A1 holds, which means that publishers are sufficiently asymmetric. We now consider the opposite case of symmetric publishers, which means that $U_{1}=U_{2}=U_{3}$, so that $U=3 U_{3}$, and $I_{12}=I_{13}=I_{23}=I$ with $I<U_{3}$. In this setting we analyze multilateral interconnection through an open platform and bilateral interconnection.

In the case of open multilateral interconnection, we need to recall that the possible interconnection profiles are $y=(0,0,0), y=(1,0,0), y=(0,1,0), y=(0,0,1)$, and $y=(1,1,1)$, but it is clear that $y=(1,0,0), y=(0,1,0)$ and $y=(0,0,1)$ are essentially equivalent when publishers are symmetric. Therefore, without loss of generality, when only two publishers are connected to the platform, we consider that they are publishers 1 and 2. Likewise, for bilateral interconnection symmetry allows to restrict attention to the interconnection profiles $y=(0,0,0), y=(1,0,0)$ [which is essentially equivalent to $y=(0,1,0)$ and to $y=(0,0,1)], y=(1,1,0)$ [which is essentially equivalent to $y=(1,0,1)$ and to $y=(0,1,1)]$, and $y=(1,1,1)$.

In this setting a result analogous to Lemma 2 holds: given any interconnection profile $y$, there exists a $\mathrm{NE}$ of pricing game $\gamma_{I I I}$ in which the library buys $\mathrm{B}_{1} \& \mathrm{~B}_{2} \& \mathrm{~B}_{3}$ and each publisher realizes a positive profit if and only if $M>M^{t}(y)=\left(2 y_{12}-y_{13}-y_{23}\right) I$. A consequence of this fact is Lemma 6 below, for which we need to make a remark related to the subgame $\gamma_{I I}$ of $\Gamma^{b}$ which follows $y=(1,1,0)$ when $M \leq M^{t}(1,1,0)=I$ : in this subgame, it is impossible that all the three publishers are active, but there is a SPNE of 
$\gamma_{I I}$ in which $A=\{1,2\}$, and a SPNE of $\gamma_{I I}$ in which $A=\{1,3\}$. In order to eliminate this ambiguity, we consider only the SPNE of $\gamma_{I I}$ such that $A=\{1,2\}$, but (again) this is only to fix the ideas.

Lemma 6 Suppose that the three publishers are symmetric. Then, for any given interconnection profile $y \in\{(0,0,0),(1,0,0),(1,1,0),(1,1,1)\}$, any SPNE of the subgame $\gamma_{I I}$ starting in stage two is such that

(i) if $M \leq M^{t}(y)$, only two publishers are active (i.e. $A^{*}=\{1,2\}$ ) and charge $P_{1}^{*}=\frac{M}{2}$, $P_{2}^{*}=\frac{M}{2}$

(ii) if $M^{t}(y)<M<U-I$, all publishers are active and charge prices such that $P_{1}^{*}=P_{1}^{t}(y)$, $P_{2}^{*}=P_{2}^{t}(y), P_{3}^{*}=P_{3}^{t}(y)$.

In particular, if $y=(1,0,0)$ then

$$
\begin{aligned}
& P_{1}^{*}=\frac{M}{2}, \quad P_{2}^{*}=\frac{M}{2}, \quad \text { when } \quad M \leq 2 I ; \\
& P_{1}^{*}=\frac{M+I}{3}, \quad P_{2}^{*}=\frac{M+I}{3}, \quad P_{3}^{*}=\frac{M-2 I}{3} \quad \text { when } \quad 2 I<M<U-I ;
\end{aligned}
$$

if instead $y=(1,1,0)$ then

$$
\begin{aligned}
& P_{1}^{*}=\frac{M}{2}, \quad P_{2}^{*}=\frac{M}{2} \quad \text { when } M \leq I ; \\
& P_{1}^{*}=\frac{M+2 I}{3}, \quad P_{2}^{*}=\frac{M-I}{3}, \quad P_{3}^{*}=\frac{M-I}{3} \quad \text { when } \quad I<M<U-I .
\end{aligned}
$$

We rely on this lemma to obtain the following proposition. ${ }^{42}$

Proposition 6 Suppose that the three publishers are symmetric. Then

(i) Under the multilateral interconnection through an open platform, for any $M$, the unique CPNE is such that all publishers are active and fully interconnected and charge $P_{1}^{*}=P_{2}^{*}=$ $P_{3}^{*}=\frac{M}{3}$.

(ii) Under bilateral interconnections;

(a) if $M \leq I$, any $C P N E$ is such that only two publishers are active (i.e. $A^{*}=\{1,2\}$ ) and they charge $P_{1}^{*}=P_{2}^{*}=\frac{M}{2}$;

(b) if instead $I<M<U-I$, the unique CPNE is such that all publishers are active and fully interconnected and charge $P_{1}^{*}=P_{2}^{*}=P_{3}^{*}=\frac{M}{3}$.

The proposition shows the robustness of the result obtained from asymmetric publishers that open multilateral interconnection leads to full interconnection while bilateral interconnection sometimes leads to partial interconnection. Precisely, for $M>I$, the two modes of interconnection generate the same full interconnection outcome, but when

\footnotetext{
${ }^{42}$ Notice that the result of Proposition 6(i) is a corollary of Proposition 2(ii).
} 
$M \leq I$, open multilateral interconnection still generates the full interconnection outcome (hence all publishers are active) whereas bilateral interconnection generates partial interconnection such that one publisher, publisher 3, is excluded because 1 and 2 gain from discriminating against him by playing $\mathbf{x}_{1}=\mathbf{x}_{2}=(1,0) .{ }^{43}$

Goyal and Moraga-Gonzales (2001) obtain a result similar to our proposition 6(ii) when they study bilateral R\&D networks among three symmetric firms. Their key parameter is a degree of knowledge spillover and they use pairwise stability. They find that the complete network is stable for all values of the parameter while one-link network is stable for the values below a threshold. Our result based on CPNE generates sharper prediction since we have a unique equilibrium outcome.

\section{Social welfare}

In our setting the sum of the publishers' profits is always equal to $M$, the library's outlay. Hence, social welfare coincides with the library's gross payoff. It follows from A1 that social welfare (as well as the library's payoff) increases with the number of bundles the library buys regardless of $y$; furthermore, for a given set of subscribed bundles, social welfare increases as more websites are interconnected. The next proposition compares different interconnection regimes in terms of social welfare.

Proposition 7 From the point of view of social welfare:

(i) In case of symmetric publishers, multilateral interconnection through an open platform (weakly) dominates bilateral interconnection.

(ii) In case of asymmetric publishers,

(a) Multilateral interconnection through an open platform (weakly) dominates bilateral interconnection when $M>M^{t}(1,1,1)$; otherwise, bilateral interconnection (weakly) dominate multilateral interconnection through an open platform.

(b) Multilateral interconnection through a for-profit platform (weakly) dominates multilateral interconnection through an open platform for any $M$.

This proposition is a straightforward consequence of Propositions 2, 3, and 5. If firms are symmetric or if asymmetry among the publishers is not too strong relative to $M$ (as in the case of $\left.M>M^{t}(1,1,1)\right)$, multilateral interconnection through an open platform maximizes social welfare. Otherwise an open platform amplifies the asymmetry, to the detriment of the small publisher, by providing free access to the interconnection technology that exhibits economies of scale. On the contrary, bilateral interconnection can create

\footnotetext{
${ }^{43}$ Notice that 1 (for instance), has no strict incentive to jointly deviate with 3 to play $x_{13}=x_{31}=1$ since anyway 1 's profit is $\frac{M}{2}$.
} 
a conflict between larger publishers such that publisher 1 may want to break connectivity with 2 while maintaining connectivity with 3 . This makes bilateral interconnection dominate multilateral interconnection through an open platform for small $M$. In a similar vein, a for-profit platform dominates an open platform for small $M$ since the former has an incentive to exploit such a conflict by discriminating against 2 and in favor of 3 when setting interconnection fees. Precisely, proposition 5 shows that inducing only 1 and 3 to interconnect is the best option for the platform when $M$ is between $M^{t}(0,1,0)$ and $M^{t}(0,0,0)$.

\section{Conclusion}

Interconnection (compatibility) among physical or virtual networks is an important issue that has been studied intensively. We contribute to the literature by studying different modes of interconnection (multilateral interconnection through an open (or a for-profit) platform, bilateral interconnection) and comparing them. One general lesson from our paper is that allowing firms to have finer instruments to discriminate interconnection results in less interconnection than when the discrimination is banned. Actually, our result that multilateral interconnection provides firms with stronger incentives to interconnect is similar to the finding of Jeon-Laffont-Tirole (2004), in a different context, that allowing telecommunication networks to discriminate between on-net calls and off-net calls can induce networks to break connectivity while without such discrimination, networks remain interconnected. In addition, both our paper and Jeon-Laffont-Tirole show that even symmetric firms may break connectivity when discrimination is allowed.

Another novel aspect of our paper is that we consider interconnection in a multihoming situation: consumers subscribe to multiple networks and seamless navigation across networks generates an added value. By contrast, the existing literature has focused on single-homing situations in which each consumer subscribes to only one among multiple networks. What drives all publishers to be fully interconnected under the open multilateral interconnection, even when they are asymmetric, is this multihoming nature combined with the convexity of the interconnection benefit which makes interconnections work as "asymmetry-amplifying forces" in our model, whereas they work as "equalizing forces" in models with single homing.

To be precise, consider three firms $i=1,2,3$ and assume that firm $i$ has a network of size $n_{i}$ before interconnection. Interconnection between $i$ and $j$ increases their network size to $n_{i}+n_{j}$; interconnection among all three makes the network size of each firm equal to $n_{1}+n_{2}+n_{3}$. Let $v(n)$, an increasing function of $n$, be the function representing the surplus a consumer obtains from a network of size $n$. In a single homing situation, if all firms are fully interconnected, a consumer obtains $v\left(n_{1}+n_{2}+n_{3}\right)$ regardless of the firm to which 
she subscribes. This is why interconnections work as "equalizing forces". If firms compete for relative standing, a dominant firm (for instance, firm 1 with $v\left(n_{1}\right)>v\left(n_{2}+n_{3}\right)$ ) has no incentive to join an open multilateral platform whereas if $v\left(n_{2}+n_{3}\right)>v\left(n_{1}\right) \geq v\left(n_{i}\right)$ for $i=2,3$, all firms join the platform.

On the contrary, interconnections work as "asymmetry-amplifying forces" in a multihoming situation if $v(n)$ is convex. ${ }^{44}$ In a multi-homing situation, a consumer cannot benefit from interconnection between $i$ and $j$ if she does not subscribe to both of them. Now consider for instance $v(n)=n^{2}$. If all three firms are interconnected, a consumer obtains $\left(n_{1}+n_{2}+n_{3}\right)^{2}$ by subscribing to all of them and obtains $\left(n_{2}+n_{3}\right)^{2}$ by subscribing to 2 and 3 ; the difference between the two terms is $n_{1}^{2}+2 n_{1}\left(n_{2}+n_{3}\right)$, which is the marginal value from subscribing to network 1 . In the absence of interconnection, this value is $n_{1}^{2}$. Therefore, interconnection increases the value from subscribing to network 1 by $2 n_{1}\left(n_{2}+n_{3}\right)$, and if $n_{1}>n_{2}>n_{3}$ then the largest firm (firm 1) benefits the most from full interconnection while the smallest (firm 3) benefits the least; this is why interconnections work as "asymmetry-amplifying forces". In particular, if firms compete for relative standing, full interconnection increases firm 1's profit and reduces firm 3's profit. In this situation it is obvious that the largest firm wants to join an open multilateral platform. Furthermore, this induces each firm $j=2,3$ to join the platform since no interconnection makes $j$ 's relative standing even worse, which leads to full interconnection.

The above argument explains well both large publishers' support for CrossRef and its success. It also suggests that CrossRef weakens competitive positions of small publishers and renders sales of their journals more difficult. In addition, the argument regarding interconnection in a single-homing situation implies that as long as there is no dominant firm, multilateral interconnection through an open platform leads to full interconnection. For instance, in the case of interconnection among internet backbone companies, the clause of no discrimination among rivals supplemented with a merger control forbidding the creation of a dominant firm would induce full interconnection, which is considered one of the four components of Internet Neutrality by Weitzer (2006). ${ }^{45}$

\section{References}

[1] Armstrong, Mark (1998). "Network Interconnection." Economic Journal, Vol. 108 , pp. $545-564$.

\footnotetext{
${ }^{44}$ In footnote 21 , we explain why $v(n)$ must be convex in our model.

${ }^{45}$ Actually, Weitzer (2006) argues that "non-discriminatory peering of backbone networks" is one of the four components of Internet Neutrality. Our clause of no discrimination among rivals is much weaker than his non-discriminatory peering, which is equivalent to mandatory full interconnection.
} 
[2] Belleflamme, Paul (2000). "Stable Coalition Structures with Open Membership and Asymmetric Firms", Games and Economic Behavior, 30: 1-21.

[3] Bernheim, Douglas, Michael Whinston and Bezalel Peleg (1987). "Coalition Proof Nash Equilibria I: Concepts" Journal of Economic Theory, 42: 1-12.

[4] Bloch, Francis and Paul Belleflamme (2004). "Market sharing agreements and stable collusive networks", International Economic Review 45: 387-411.

[5] Bolman, Pieter S. H. (2002). "The Consortium Site Licence: Unfinished Business?" The Consortium Site Licence: Is It a Sustainable Model? Ingenta Institute, 241-246.

[6] Cabrales, Antonio and Antoni Calvò-Armengol (2007). "Corporate Downsizing to rebuild team spirit: how costly voting can foster competition", Journal of European Economic Association 5: 1016-1042.

[7] Church, Jeffrey and Neil Gandal (2005). "Platform Competition in Telecommunications", in The Handbook of Telecommunications Economics, Volume 2 edited by M. Cave, S. Majumdar, and I. Vogelsang, Elsevier.

[8] Crémer, Jacques, Patrick Rey and Jean Tirole.(2000) "Connectivity in the Commercial Network" Journal of Industrial Economics, 48(4): 433-72

[9] M. Dewatripont, V. Ginsburgh, P. Legros, A. Walckiers, J.-P. Devroey, M. Dujardin, F. Vandooren, P. Dubois, J. Foncel, M. Ivaldi, M.-D. Heusse (2006). Study on the Economic and Technical Evolution of the Scientific Publication Markets in Europe.

[10] Dutta, Bhaskar and Suresh Mutuswami (1997). "Stable Networks." Journal of Economic Theory, 76: 322-344.

[11] Dutta, Bhaskar, Anne van den Nouweland and Stef Tijs (1998). "Link formation in cooperative situations." International Journal of Game Theory, 27: 245-256.

[12] Economides, Nicholas (2006). "The Economics of the Internet Backbone," Handbook of Telecommunications, Amsterdam: Elsevier Publishers, 373-412

[13] Edlin, Aaron S. and Daniel L. Rubinfeld (2004). "Exclusion or Efficient Pricing: The "Big Deal" Bundling of Academic Journals", Antitrust Law Journal, 72(1): 119-157

[14] European Commission (2007). Conference Proceedings of "Scientific Publishing in the European Research Area: Access, Dissemination and Preservation in the Digital Area". 
[15] Farrell, Joseph and Garth Saloner (1985). "Standardization, Compatibility, and Innovation", The RAND Journal of Economics, 16, 70-83.

[16] Farrell, Joseph and Garth Saloner (1986). "Installed Base and Compatibility: Innovation, Product Preannouncements and Predation", American Economic Review, 76, 940-955.

[17] Goyal, Sanjeev (2007). Connections: an introduction to the economics of networks, Princeton University Press.

[18] Goyal, Sanjeev, Alexander Konovalov and José Luis Moraga-Gonzales (2008), "Hybrid R\&D" Journal of the European Economic Association, 6: 1309-1338.

[19] Goyal, Sanjeev and José Luis Moraga-Gonzales (2001), "R\&D Networks" Rand Journal of Economics 32: 686-707.

[20] Haank, Derk (2001). "Is Electronic Publishing Being Used in the Best Interests of Science? The Publisher's View." Proceedings of the Second ICSU-UNESCO International Conference on Electronic Publishing in Science.

[21] Jackson, Matthew O. and Asher Wolinsky (1996). "A Strategic Model of Social and Economic Networks." Journal of Economic Theory, 71: 44-74.

[22] Jackson, Matthew O. (2008) Social and Economic Networks, Princeton University Press.

[23] Jeon, Doh-Shin, Jean-Jacques Laffont and Jean Tirole (2004). "On the "receiverpays" Principle," Rand Journal of Economics, 35(1): 85-110.

[24] Jeon, Doh-Shin and Domenico Menicucci (2006). "Bundling Electronic Journals and Competition among Publishers", Journal of the European Economic Association, September 4(5): 1038-83.

[25] Jeon, Doh-Shin and Domenico Menicucci (2009). "Interconnection among Academic Journal Websites: Multilateral versus Bilateral Interconnection", working paper, Toulouse School of Economics

[26] Katz, Michael L. and Carl Shapiro (1985), "Network Externalities, Competition, and Compatibility", American Economic Review, 75, 424-440.

[27] Key Perspectives (2002). "Library Consortia Research: Library and Publisher Studies." The Consortium Site Licence: Is It a Sustainable Model? Ingenta Institute, 80-118. 
[28] Laffont, Jean-Jacques, Scott Marcus, Patrick Rey and Jean Tirole (2003), "Internet Interconnection and the Off-Net-Cost Pricing Principle", Rand Journal of Economics, 34: $370-390$

[29] Laffont, Jean-Jacques, Patrick Rey and Jean Tirole (1998a). "Network Competition I: Overview and Nondiscriminatory Pricing." Rand Journal of Economics, 29: 1-37.

[30] Laffont, Jean-Jacques, Patrick Rey and Jean Tirole (1998b). "Network Competition II: Price Discrimination." Rand Journal of Economics, 29: 38-56.

[31] Matutes, Carmen and A. Jorge Padilla (1994). "Shared ATM Networks and Banking Competition." European Economic Review, 38: 1113-1138.

[32] McCabe, Mark (2004). "Information Goods and Endogenous Pricing Strategies: the Case of Academic Journals." Economics Bulletin, vol. 12, No. 10

[33] Myerson, Roger (1991). Game Theory, Harvard University Press.

[34] Simon, H. A. (1971), "Designing Organizations for an Information-Rich World", written at Baltimore, MD, in Martin Greenberger, Computers, Communication, and the Public Interest, The Johns Hopkins Press, ISBN 0-8018-1135-X.

[35] U.K. Competition Commission (2001). Reed Elsevier Plc and Harcourt General, Inc: A report on the proposed merger, London, U.K., available at http://www.competitioncommission.org.uk/rep_pub/reports/2001/457reed.htm\#full

[36] Yi, Sang-Seung (1998), "Endogenous Formation of Joint Ventures with Efficiency Gains." Rand Journal of Economics, 29: 610-631.

[37] Yi, Sang-Seung and Hyukseung Shin (2000), "Endogenous Formation of Research Coalitions with Spillovers." International Journal of Industrial Organization, 18: 229-256.

[38] Weitzner, Daniel J. (2006), "The Neutral Internet: An Information Architecture for Open Societies", available at http://dig.csail.mit.edu/2006/06/neutralnet.html

\section{Appendix}

Before starting the proofs, it is useful to introduce a new piece of notation. For any non-empty subset $Z$ of $\{1,2,3\}$, let $u(Z)$ denote the library's payoff when she buys the bundles $\left\{\mathrm{B}_{i}: i \in Z\right\}$. For instance, $u(1)=U_{1}-P_{1}$ and $u(13)=U_{1}+U_{3}+I_{13} y_{13}-P_{1}-P_{3}$. 


\subsection{Proof of Lemma 1}

\subsubsection{Step 1: 1 is always active}

Proof. Given an arbitrary $y$, we show that 1 is active in any SPNE of $\gamma_{I I}$ because he earns a profit in any NE of $\gamma_{I I I}$ if he is active. Precisely, we prove that if $A=\{1,2,3\}$ then there exists no NE of $\gamma_{I I I}$ in which 1 makes zero profit. ${ }^{46}$ Let $\left(P_{1}^{*}, P_{2}^{*}, P_{3}^{*}\right)$ represent the equilibrium of the subgame starting from stage 3 when $A=\{1,2,3\}$. We below show that if $\left(P_{1}^{*}, P_{2}^{*}, P_{3}^{*}\right)$ is such that 1 earns zero, then 1 can make a profit by playing $P_{1}>0$ close to zero, given A1 which implies $U_{1} \geq U_{2}+I_{23}$. We start by observing that if $\left(P_{1}^{*}, P_{2}^{*}, P_{3}^{*}\right)$ is such that 1 earns no profit, then it is necessary that the library is spending $M$ in aggregate in buying $\mathrm{B}_{2}$ and/or $\mathrm{B}_{3}$, because if the library spends less than $M$ then it is obvious that 1 would succeed in selling $B_{1}$ at a small positive price. We need to distinguish three non-obvious cases.

Both 2 and 3 make a profit with $\left(P_{1}^{*}, P_{2}^{*}, P_{3}^{*}\right)$ Then $P_{1}>0$ close to zero implies that $u(1)>\max \{u(2), u(3)\}$ and $u(12) \simeq U_{1}+U_{2}+I_{12} y_{12}-P_{2}^{*}>u(23)=U_{2}+U_{3}+$ $I_{23} y_{23}-P_{2}^{*}-P_{3}^{*}$ since $U_{1}+I_{12} y_{12}>U_{3}+I_{23} y_{23}$. Therefore the library buys either $\mathrm{B}_{1}$, or $\mathrm{B}_{1} \& \mathrm{~B}_{2}$, or $\mathrm{B}_{1} \& \mathrm{~B}_{3}$ and 1 earns $P_{1}>0$.

Only 2 makes a profit with $\left(P_{1}^{*}, P_{2}^{*}, P_{3}^{*}\right)$ This requires $P_{2}^{*}=M$ and we consider first the case of $P_{3}^{*}>0$. Then $P_{1}>0$ close to zero implies that $\mathrm{B}_{1} \& \mathrm{~B}_{2}$ and $\mathrm{B}_{2} \& \mathrm{~B}_{3}$ are unaffordable for the library and $u(1)>\max \{u(2), u(3)\}$; therefore the library buys either $\mathrm{B}_{1}$ or $\mathrm{B}_{1} \& \mathrm{~B}_{3}$ and 1 earns $P_{1}>0$. If instead $P_{3}^{*}=0$, then $P_{1}>0$ close to zero implies that $\mathrm{B}_{1} \& \mathrm{~B}_{2}$ is unaffordable for the library, $u(13)>\max \{u(1), u(3)\}, u(23)>u(2)$ and $u(13) \simeq U_{1}+U_{3}+I_{13} y_{13}>u(23)=U_{2}+U_{3}+I_{23} y_{23}-M$ since $U_{1}+I_{13} y_{13} \geq U_{2}+I_{23} y_{23} ;$ therefore the library buys $\mathrm{B}_{1} \& \mathrm{~B}_{3}$ and 1 earns $P_{1}>0$.

Only 3 makes a profit with $\left(P_{1}^{*}, P_{2}^{*}, P_{3}^{*}\right)$ This requires $P_{3}^{*}=M$ and we consider first the case of $P_{2}^{*}>0$. Then $P_{1}>0$ close to zero implies that $\mathrm{B}_{1} \& \mathrm{~B}_{3}$ and $\mathrm{B}_{2} \& \mathrm{~B}_{3}$ are unaffordable and $u(1)>\max \{u(2), u(3)\}$; therefore the library buys either $\mathrm{B}_{1}$ or $\mathrm{B}_{1} \& \mathrm{~B}_{2}$. If instead $P_{2}^{*}=0$, then $P_{1}>0$ close to zero implies that $\mathrm{B}_{1} \& \mathrm{~B}_{3}$ is unaffordable, $u(12)>$ $\max \{u(1), u(2)\}, u(23)>u(3)$ and $u(12) \simeq U_{1}+U_{2}+I_{12} y_{12}>u(23)=U_{2}+U_{3}+I_{23} y_{23}-M$ since $U_{1}+I_{12} y_{12}>U_{3}+I_{23} y_{23}$.

\footnotetext{
${ }^{46}$ In the case of $A=\{1,2\}$ or $A=\{1,3\}$ the proof is straightforward: it suffices that 1 plays $P_{1}<U_{1}-U_{2}$ if $A=\{1,2\}$ and $P_{1}<U_{1}-U_{3}$ if $A=\{1,3\}$.
} 


\subsubsection{Step 2: 2 is active if 3 is active}

Proof. Given an arbitrary $y$ we prove that there exists no SPNE of $\gamma_{I I}$ in which $A=$ $\{1,3\}$. In such a case it is necessary that $M>U_{1}-U_{3}$ because if $M \leq U_{1}-U_{3}$, then 3 cannot make any profit: the library prefers to buy $\mathrm{B}_{1}$ at $P_{1}=M$ rather than $\mathrm{B}_{3}$ at any $P_{3}>0$. Given that $M>U_{1}-U_{3}, 2$ can profitably deviate by becoming active. Precisely, we prove that if $A=\{1,2,3\}$ and $M>U_{1}-U_{3}$ then there exists no NE of $\gamma_{I I I}$ in which 2 makes zero profit. This result is obtained by showing that if $\left(P_{1}^{*}, P_{2}^{*}, P_{3}^{*}\right)$ is such that 2 earns zero, then he can make a profit by playing $P_{2}>0$ close to zero given A1 which implies $U_{2} \geq U_{3}+I_{13}$. We start by observing that if $\left(P_{1}^{*}, P_{2}^{*}, P_{3}^{*}\right)$ is such that 2 earns no profit, then it is necessary that the library is spending $M$ in aggregate in buying $\mathrm{B}_{1}$ and/or $B_{3}$, otherwise it is obvious that 2 would succeed in selling $B_{2}$ at a small price. Since we have shown above that 1 makes a profit in any NE of $\gamma_{I I I}$, we need to consider only two non-obvious cases.

Both 1 and 3 make a profit with $\left(P_{1}^{*}, P_{2}^{*}, P_{3}^{*}\right) \quad$ This implies $u(13) \geq \max \{u(1), u(3)\}$, and $P_{2}>0$ close to zero implies that $u(12) \simeq U_{1}+U_{2}+I_{12} y_{12}-P_{1}^{*}>u(13)=U_{1}+U_{3}+$ $I_{13} y_{13}-P_{1}^{*}-P_{3}^{*}$ since $U_{2}+I_{12} y_{12} \geq U_{3}+I_{13} y_{13}$. Therefore the library buys either $\mathrm{B}_{2}$, or $\mathrm{B}_{1} \& \mathrm{~B}_{2}$, or $\mathrm{B}_{2} \& \mathrm{~B}_{3}$ and 2 earns $P_{2}>0$.

Only 1 makes a profit with $\left(P_{1}^{*}, P_{2}^{*}, P_{3}^{*}\right)$ This requires $P_{1}^{*}=M$ and we consider first the case of $P_{3}^{*}>0$. Then $P_{2}>0$ close to zero implies that $\mathrm{B}_{1} \& \mathrm{~B}_{2}$ and $\mathrm{B}_{1} \& \mathrm{~B}_{3}$ are unaffordable for the library and $u(2)>\max \{u(1), u(3)\}$ since $M>U_{1}-U_{3}>U_{1}-U_{2}$. Therefore the library buys either $\mathrm{B}_{2}$ or $\mathrm{B}_{2} \& \mathrm{~B}_{3}$ and 2 earns $P_{2}>0$. If instead $P_{3}^{*}=0$, then $P_{2}>0$ close to zero implies that $\mathrm{B}_{1} \& \mathrm{~B}_{2}$ is unaffordable and $u(23)>\max \{u(2), u(3)\}$, $u(13)>u(1)$ and $u(23) \simeq U_{2}+U_{3}+I_{23} y_{23}, u(13)=U_{1}+U_{3}+I_{13} y_{13}-M$; the inequality $u(23)>u(13)$ holds since $M>U_{1}-U_{3}$ and $U_{2}+I_{23} y_{23} \geq U_{3}+I_{13} y_{13}$ and thus we conclude that the library buys $\mathrm{B}_{2} \& \mathrm{~B}_{3}$.

\subsection{Proof of Lemma 2}

Let $\left(P_{1}^{*}, P_{2}^{*}, P_{3}^{*}\right)$ be the equilibrium prices. We start by proving that

$$
P_{1}^{*}+P_{2}^{*}+P_{3}^{*}=M
$$

If $P_{1}^{*}+P_{2}^{*}+P_{3}^{*}<M$, then $M<U$ implies that $P_{i}^{*}<U_{i}$ for at least one $i$ and then a profitable deviation for $i$ exists. Indeed, the library would still be willing to buy $\mathrm{B}_{1} \& \mathrm{~B}_{2} \& \mathrm{~B}_{3}$ even if $P_{i}$ were larger than $P_{i}^{*}$, provided that $P_{i}<U_{i}$ and $P_{i}+P_{j}^{*}+P_{j}^{*} \leq M$.

In order to determine the other conditions which must be satisfied by $\left(P_{1}^{*}, P_{2}^{*}, P_{3}^{*}\right)$, we notice that if 1 increases $P_{1}$ above $P_{1}^{*}$, the library cannot afford to buy anymore $\mathrm{B}_{1} \& \mathrm{~B}_{2} \& \mathrm{~B}_{3}$. 
Thus, the deviation of 1 is profitable for 1 if and only if the library buys $B_{1}$, or $B_{1} \& B_{2}$, or $\mathrm{B}_{1} \& \mathrm{~B}_{3}$. Therefore, there is no profitable deviation for 1 if and only if

$$
\max \{0, u(2), u(3), u(23)\} \geq \max \{u(1), u(12), u(13)\}
$$

is satisfied at prices $\left(P_{1}^{*}, P_{2}^{*}, P_{3}^{*}\right)$. This condition means that if $P_{1}$ is increased above $P_{1}^{*}$, then the best bundle for the library among $\mathrm{B}_{1}, \mathrm{~B}_{1} \& \mathrm{~B}_{2}, \mathrm{~B}_{1} \& \mathrm{~B}_{3}$, is strictly worse than the best alternative among $\mathrm{B}_{2}, \mathrm{~B}_{3}, \mathrm{~B}_{2} \& \mathrm{~B}_{3}$, and no purchase. Conditions (9)-(10), which need to be satisfied at prices $\left(P_{1}^{*}, P_{2}^{*}, P_{3}^{*}\right)$, are analogous to $(8)$ in that they guarantee that there is no profitable deviation for firms 2 and 3 :

$$
\begin{aligned}
& \max \{0, u(1), u(3), u(13)\} \geq \max \{u(2), u(12), u(23)\} \\
& \max \{0, u(1), u(2), u(12)\} \geq \max \{u(3), u(13), u(23)\}
\end{aligned}
$$

A useful property is that in (8), (9), and (10), the left hand side is strictly positive. Indeed, if the left hand side in (9) - for instance - is zero, then the right hand side is not larger than zero. Therefore, we have $u(1) \leq 0, u(2) \leq 0$, and $u(3) \leq 0$, that is $P_{1}^{*} \geq U_{1}$, $P_{2}^{*} \geq U_{2}$, and $P_{3}^{*} \geq U_{3}$. These three inequalities, given (7), imply $M \geq U$, which violates the assumption $M<U-I_{12}$. Therefore, (8)-(10) can be written as

$$
\begin{aligned}
& \max \{u(2), u(3), u(23)\} \geq \max \{u(1), u(12), u(13)\} \\
& \max \{u(1), u(3), u(13)\} \geq \max \{u(2), u(12), u(23)\} \\
& \max \{u(1), u(2), u(12)\} \geq \max \{u(3), u(13), u(23)\}
\end{aligned}
$$

In the following we distinguish three cases, depending on the value of $\max \{u(2), u(3), u(23)\}$.

The case in which the left hand side of (11) is $u(2)$ From $u(2) \geq u(23)$ it follows that

$$
P_{3} \geq U_{3}+I_{23} y_{23}
$$

and $u(3) \leq 0$. With reference to $(12)$, we need to distinguish the case of $u(1) \geq u(13)$ from the case of $u(13)>u(1)$.

If $u(1) \geq u(13)$, then $u(2) \geq u(1) \geq u(2)$ by (11) and (12). Thus $u(1)=u(2)>0$ and $u(12)=u(1)+u(2)+I_{12} y_{12}>u(1)=u(2)$, which violates (11): contradiction.

If instead $u(13)>u(1)$, we notice that (14) implies $y_{13}=1$ (and $P_{3}<U_{3}+I_{13}$ ). Then (11)-(13) boil down to $u(2)=u(13) \geq u(12)$, which means that equilibrium prices satisfy

$$
U_{2}-P_{2}=U_{1}+U_{3}+I_{13}-P_{1}-P_{3}, \quad P_{1}+P_{2}+P_{3}=M
$$

This yields $P_{2}=U_{2}+\frac{1}{2}\left(M-U-I_{13}\right), P_{1}+P_{3}=\frac{1}{2}\left(M+U+I_{13}\right)-U_{2}$ and $u(13)=u(2)=$ $\frac{1}{2}\left(U+I_{13}-M\right)$. Furthermore, $u(12)=U_{1}+U_{2}+I_{12} y_{12}-\left(M-P_{3}\right)$ and thus $u(2) \geq u(12)$ reduces to

$$
M \geq U-2 U_{3}+2 I_{12} y_{12}-I_{13}+2 P_{3}
$$


Since $P_{3} \geq U_{3}$ by (14), we see that (15) requires $M \geq U-I_{13}>U-I_{12}$, a contradiction.

The case in which the left hand side of (11) is $u(3)$ From $u(3) \geq u(23)$ it follows that

$$
P_{2} \geq U_{2}+I_{23} y_{23}
$$

and $u(2) \leq 0$. With reference to (13), we need to distinguish the case of $u(1) \geq u(12)$ from the case of $u(12)>u(1)$.

If $u(1) \geq u(12)$, then $u(3) \geq u(1) \geq u(3)$ by (11) and (13). Thus $u(1)=u(3)>0$ and $u(13)>u(1)=u(3)$, which violates $(11)$.

If instead $u(12)>u(1)$, we notice that (16) implies $y_{12}=1$ (and $P_{2}<U_{2}+I_{12}$ ). Then (11)-(13) boil down to $u(3)=u(12) \geq u(13)$, which means that equilibrium prices satisfy

$$
U_{3}-P_{3}=U_{1}+U_{2}+I_{12}-P_{1}-P_{2}, \quad P_{1}+P_{2}+P_{3}=M
$$

This yields $P_{3}=U_{3}+\frac{1}{2}\left(M-U-I_{12}\right), P_{1}+P_{2}=\frac{1}{2}\left(M+U+I_{12}\right)-U_{3}$ and thus $u(3)=u(12)=\frac{1}{2}\left(U+I_{12}-M\right)$. Furthermore, $u(13)=U_{1}+U_{3}+I_{13} y_{13}-\left(M-P_{2}\right)$ and then $u(3) \geq u(13)$ reduces to

$$
M \geq U-2 U_{2}+2 I_{13} y_{13}-I_{12}+2 P_{2}
$$

Since $P_{2} \geq U_{2}+I_{23} y_{23}$ by (16), we see that (17) implies $M \geq U-I_{12}$, a contradiction.

The case in which the left hand side of (11) is $u(23) \quad$ From $u(23) \geq \max \{u(2), u(3)\}$ it follows that $P_{2} \leq U_{2}+I_{23} y_{23}$ and $P_{3} \leq U_{3}+I_{23} y_{23}$, but we can restrict our attention to the case in which

$$
P_{2}<U_{2}+I_{23} y_{23}, \quad P_{3}<U_{3}+I_{23} y_{23}
$$

since otherwise the left hand side of $(11)$ is $\max \{u(2), u(3)\}$, and we have already considered this possibility. Therefore (11)-(13) reduce to [notice that (19) is used to obtain (20)-(21)]:

$$
\begin{aligned}
u(23) & \geq \max \{u(1), u(12), u(13)\} \\
\max \{u(1), u(13)\} & \geq u(23) \\
\max \{u(1), u(12)\} & \geq u(23)
\end{aligned}
$$

With reference to $(20)$, we need to distinguish the case in which $u(1) \geq u(13)$ from the case in which $u(13)>u(1)$. In the first case we find

$$
P_{3} \geq U_{3}+I_{13} y_{13}
$$


and thus $y_{13}=0, y_{23}=1$ needs to hold since otherwise (18) is violated. Furthermore, (19)-(21) reduce to $u(23)=u(1) \geq \max \{u(12), u(13)\}$ and prices satisfy

$$
U_{1}-P_{1}=U_{2}+U_{3}+I_{23}-P_{2}-P_{3}, \quad P_{1}+P_{2}+P_{3}=M
$$

The two equalities yield $P_{1}=U_{1}+\frac{1}{2}\left(M-U-I_{23}\right), P_{2}+P_{3}=\frac{1}{2}\left(M+U+I_{23}\right)-U_{1}$ and $u(1)=u(23)=\frac{1}{2}\left(U+I_{23}-M\right)$. Furthermore, $u(12)=U_{1}+U_{2}+I_{12} y_{12}-\left(M-P_{3}\right)$ and $u(1) \geq u(12)$ reduces to

$$
M \geq U-2 U_{3}+2 I_{12} y_{12}-I_{23}+2 P_{3}
$$

and then (22) and (23) imply $M>U-I_{12}$, a contradiction.

In the case in which $u(13)>u(1)$ we find that $u(13)=u(23)=u(12)$, which reduce to (1)-(2); using $P_{1}^{*}+P_{2}^{*}+P_{3}^{*}=M$ we obtain $P_{1}^{*}=P_{1}^{t}(y), P_{2}^{*}=P_{2}^{t}(y), P_{3}^{*}=P_{3}^{t}(y)$. Notice that $P_{1}^{t}(y)>P_{2}^{t}(y)>P_{3}^{t}(y)$ for any $y$ (given A1), and $P_{3}^{t}(y)>0$ if and only if $M>M^{t}(y)$. Furthermore, the conditions $u(23)>\max \{u(2), u(3)\}$ and $u(13)>u(1)$ are equivalent, respectively, to $U+2 I_{12} y_{12}-I_{13} y_{13}+2 I_{23} y_{23}>M, U-I_{12} y_{12}+2 I_{13} y_{13}+2 I_{23} y_{23}>M$ and $U+2 I_{12} y_{12}+2 I_{13} y_{13}-I_{23} y_{23}>M$, which are satisfied since $M<U-I_{12}$.

\subsection{Proof of Lemma 3}

We know that publisher 1 is active for any $M$, given Lemma 1 .

Consider the case in which $M>M^{t}(y)$. Then publisher 2 is certainly active, as that yields him either $P_{2}^{t}(y)>0$ (if also publisher 3 is active) or $P_{2}^{d}>0$ (if publisher 3 is not active). Furthermore, also publisher 3 is active as that yields him $P_{3}^{t}(y)>0$.

Consider the case in which $U_{1}-U_{2}<M \leq M^{t}(y)$. Then $A^{*} \neq\{1,2,3\}$, as Lemma 2 implies that there exists no NE in $\gamma_{I I I}$ in which all three publishers make a profit. Also, $A^{*} \neq\{1\}$ as 2 can make a profit $P_{2}^{d}>0$ by entering; thus $A^{*}=\{1,2\}$.

Finally, in the case in which $M \leq U_{1}-U_{2}$ we obtain $A^{*}=\{1\}$, since there is no room for publisher 2 nor for publisher 3 to make a profit.

\subsection{Proof of Lemma 4}

Consider first the case of $M$ between $M^{t}(0,1,0)$ and $M^{t}(0,0,0)$, and notice that $G_{1,3}^{m}(0)$ is defined as follows:

$$
\begin{array}{rc|c|c|} 
& \multicolumn{1}{c}{1 \backslash 3} & x_{3}=0 & x_{3}=1 \\
\cline { 3 - 4 } G_{1,3}^{m}(0): & x_{1}=0 & P_{1}^{d}, 0 & P_{1}^{d}, 0 \\
\cline { 3 - 4 } & x_{1}=1 & P_{1}^{d}, 0 & P_{1}^{t}(0,1,0), P_{3}^{t}(0,1,0) \\
\cline { 3 - 4 } & &
\end{array}
$$

In this game, $\left(x_{1}, x_{3}\right)=(0,0)$ is a NE [otherwise $x=(0,0,0)$ would not be a NE in $G^{m}$ ]. But since $P_{1}^{t}(0,1,0)>P_{1}^{d}$ and $P_{3}^{t}(0,1,0)>0$ hold for any $M$ such that $M^{t}(0,1,0)<M \leq$ 
$M^{t}(0,0,0),\left(x_{1}, x_{3}\right)=(1,1)$ is also a NE of $G_{1,3}^{m}(0)$. Furthermore, $\left(x_{1}, x_{3}\right)=(1,1)$ strictly Pareto dominates $\left(x_{1}, x_{3}\right)=(0,0)$ for publishers 1 and 3 , that is $P_{1}^{t}(0,1,0)>P_{1}^{d}$ and $P_{3}^{t}(1,0,1)>0$. This implies that condition (ii) in Definition 1 is not satisfied when $x=(0,0,0)$, and therefore this profile is not a CPNE for $M^{t}(0,1,0)<M \leq M^{t}(0,0,0)$. The case of $M^{t}(0,0,0)<M \leq M^{t}(1,0,0)$ is considered in the main text just after the statement of the lemma. In the case of $M>M^{t}(1,0,0), G_{1,2}^{m}(0)$ is defined as follows:

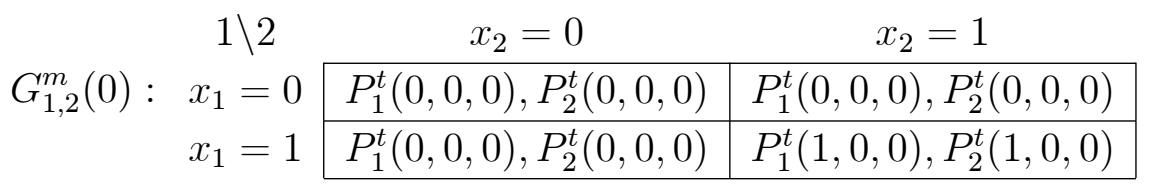

Here we have $P_{1}^{t}(1,0,0)>P_{1}^{t}(0,0,0)$ and $P_{2}^{t}(1,0,0)>P_{2}^{t}(0,0,0)$, and thus we can argue like above to conclude that $x=(0,0,0)$ is not a CPNE.

\subsection{Proof of Proposition 2 under A1}

We first present the proof of proposition 2 under A1 and then give the proof for when A1 does not hold but $\mathrm{A} 1^{w}$ holds.

It is simple to see that if $M \leq M^{t}(0,1,0)$, then interconnection is irrelevant since, for any $y$, either only 1 is active (if $M \leq U_{1}-U_{2}$ ), or only 1 and 2 are active [if $U_{1}-U_{2}<$ $\left.M \leq M^{t}(0,1,0)\right]$. Thus $\pi_{1}, \pi_{2}, \pi_{3}$ do not depend on $x$ for $M \leq M^{t}(0,1,0), x=(1,1,1)$ is a CPNE of $G^{m}$ and any $x \neq(1,1,1)$ generates the same outcome. The rest of the proof considers the case in which $M>M^{t}(0,1,0)$ and is split in four steps.

\subsubsection{Step 1 For each publisher $i$, playing $x_{i}=1$ weakly dominates $x_{i}=0$}

Proof. Using Lemma 3 we consider the various profiles $\left(x_{j}, x_{k}\right)$ which rival publishers may play, except $\left(x_{j}, x_{k}\right)=(0,0)$ since then $x_{i}$ has no effect, and examine how $x_{i}$ affects $i$ 's profit.

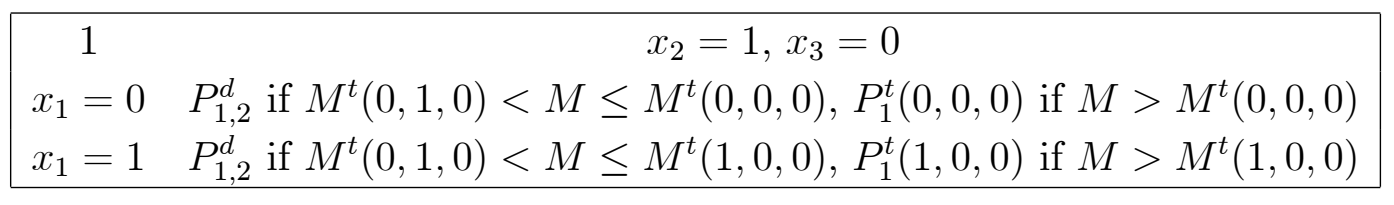

\begin{tabular}{|c|c|}
\hline $\begin{array}{c}1 \\
x_{1}=0 \\
x_{1}=1\end{array}$ & $\begin{array}{c}x_{2}=1, x_{3}=1 \\
P_{1,2}^{d} \text { if } M^{t}(0,1,0)<M \leq M^{t}(0,0,1), P_{1}^{t}(0,0,1) \text { if } M>M^{t}(0,0,1) \\
P_{1,2}^{d} \text { if } M^{t}(0,1,0)<M \leq M^{t}(1,1,1), P_{1}^{t}(1,1,1) \text { if } M>M^{t}(1,1,1)\end{array}$ \\
\hline
\end{tabular}

$$
\begin{aligned}
& \begin{array}{|cc|}
\hline 1 & x_{2}=0, x_{3}=1 \\
x_{1}=0 & P_{1,2}^{d} \text { if } M^{t}(0,1,0)<M \leq M^{t}(0,0,0), P_{1}^{t}(0,0,0) \text { if } M>M^{t}(0,0,0) \\
x_{1}=1 & P_{1}^{t}(0,1,0) \text { for any } M>M^{t}(0,1,0) \\
\hline
\end{array}
\end{aligned}
$$


In order to see that $x_{1}=1$ weakly dominates $x_{1}=0$ for 1 , notice that $M^{t}(0,0,0)<$ $M^{t}(1,0,0)$ and $P_{1,2}^{d}>P_{1}^{t}(0,0,0)$ for $M$ between $M^{t}(0,0,0)$ and $M^{t}(1,0,0), P_{1}^{t}(1,0,0)>$ $P_{1}^{t}(0,0,0)$ for $M>M^{t}(1,0,0) ; M^{t}(0,1,0)<M^{t}(0,0,0)$ and $P_{1}^{t}(0,1,0)>P_{1,2}^{d}$ for $M$ between $M^{t}(0,1,0)$ and $M^{t}(0,0,0), P_{1}^{t}(0,1,0)>P_{1}^{t}(0,0,0)$ for $M>M^{t}(0,0,0) ; M^{t}(0,0,1)<$ $M^{t}(1,1,1)$ and $P_{1,2}^{d}>P_{1}^{t}(0,0,1)$ for $M$ between $M^{t}(0,0,1)$ and $M^{t}(1,1,1), P_{1}^{t}(1,1,1)>$ $P_{1}^{t}(0,0,1)$ for $M>M^{t}(1,1,1)$.

\begin{tabular}{|c|c|}
\hline $\begin{aligned} 2 & \\
x_{2} & =0 \\
x_{2} & =1\end{aligned}$ & $\begin{array}{c}x_{1}=1, x_{3}=0 \\
P_{2,1}^{d} \text { if } M^{t}(0,1,0)<M \leq M^{t}(0,0,0), P_{2}^{t}(0,0,0) \text { if } M>M^{t}(0,0,0) \\
P_{2,1}^{d} \text { if } M^{t}(0,1,0)<M \leq M^{t}(1,0,0), P_{2}^{t}(1,0,0) \text { if } M>M^{t}(1,0,0)\end{array}$ \\
\hline $\begin{aligned} 2 & \\
x_{2} & =0 \\
x_{2} & =1\end{aligned}$ & $\begin{array}{c}x_{1}=0, x_{3}=1 \\
P_{2,1}^{d} \text { if } M^{t}(0,1,0)<M \leq M^{t}(0,0,0), P_{2}^{t}(0, \\
P_{2,1}^{d} \text { if } M^{t}(0,1,0)<M \leq M^{t}(0,0,1), P_{2}^{t}(0,\end{array}$ \\
\hline $\begin{array}{c}2 \\
x_{2}=0 \\
x_{2}=1\end{array}$ & $\begin{array}{r}x_{1}=1, \\
P_{2}^{t}(0,1,0) \text { for any } \\
P_{2,1}^{d} \text { if } M^{t}(0,1,0)<M \leq M^{t}(1,1,\end{array}$ \\
\hline
\end{tabular}

In order to see that $x_{2}=1$ weakly dominates $x_{2}=0$ for 2 , notice that $M^{t}(0,0,0)<$ $M^{t}(1,0,0)$ and $P_{2,1}^{d}>P_{2}^{t}(0,0,0)$ for $M>M^{t}(0,0,0), P_{2}^{t}(1,0,0)>P_{2}^{t}(0,0,0)$ for $M>$ $M^{t}(1,0,0) ; M^{t}(0,0,1)<M^{t}(0,0,0)$ and $P_{2}^{t}(0,0,1)>P_{2,1}^{d}$ for $M$ between $M^{t}(0,0,1)$ and $M^{t}(0,0,0), P_{2}^{t}(0,0,1)>P_{2}^{t}(0,0,0)$ for $M>M^{t}(0,0,0) ; M^{t}(0,1,0)<M^{t}(1,1,1)$ and $P_{2,1}^{d}>P_{2}^{t}(0,1,0)$ for $M$ between $M^{t}(0,1,0)$ and $M^{t}(1,1,1), P_{2}^{t}(1,1,1)>P_{2}^{t}(0,1,0)$ for $M>M^{t}(1,1,1)$.

\begin{tabular}{|c|c|}
\hline $\begin{array}{c}3 \\
x_{3}=0 \\
x_{3}=1\end{array}$ & $\begin{array}{c}x_{1}=1, x_{2}=0 \\
0 \text { if } M^{t}(0,1,0)<M \leq M^{t}(0,0,0), P_{3}^{t}(0,0,0) \text { if } M>M^{t}(0,0,0) \\
P_{3}^{t}(0,1,0) \text { for any } M>M^{t}(0,1,0)\end{array}$ \\
\hline $\begin{array}{c}3 \\
x_{3}=0 \\
x_{3}=1\end{array}$ & $\begin{array}{c}x_{1}=0, x_{2}=1 \\
0 \text { if } M^{t}(0,1,0)<M \leq M^{t}(0,0,0), P_{3}^{t}(0,0,0) \text { if } M>M^{t}(0,0,0) \\
0 \text { if } M^{t}(0,1,0)<M \leq M^{t}(0,0,1), P_{3}^{t}(0,0,1) \text { if } M>M^{t}(0,0,1)\end{array}$ \\
\hline $\begin{array}{c}3 \\
x_{3}=0 \\
x_{3}=1\end{array}$ & $\begin{array}{c}x_{1}=1, x_{2}=1 \\
0 \text { if } M^{t}(0,1,0)<M \leq M^{t}(1,0,0), P_{3}^{t}(1,0,0) \text { if } M>M^{t}(1,0,0) \\
0 \text { if } M^{t}(0,1,0)<M \leq M^{t}(1,1,1), P_{3}^{t}(1,1,1) \text { if } M>M^{t}(1,1,1)\end{array}$ \\
\hline
\end{tabular}

In order to see that $x_{3}=1$ weakly dominates $x_{3}=0$ for 3 , notice that $M^{t}(0,1,0)<$ $M^{t}(0,0,0)$ and $P_{3}^{t}(0,1,0)>0$ for $M$ between $M^{t}(0,1,0)$ and $M^{t}(0,0,0), P_{3}^{t}(0,1,0)>$ $P_{3}^{t}(0,0,0)$ for $M>M^{t}(0,0,0) ; M^{t}(0,0,1)<M^{t}(0,0,0)$ and $P_{3}^{t}(0,0,1)>0$ for $M$ between $M^{t}(0,0,1)$ and $M^{t}(0,0,0), P_{3}^{t}(0,0,1)>P_{3}^{t}(0,0,0)$ for $M>M^{t}(0,0,0) ; M^{t}(1,1,1)<$ $M^{t}(1,0,0)$ and $P_{3}^{t}(1,1,1)>0$ for $M$ between $M^{t}(1,1,1)$ and $M^{t}(1,0,0), P_{3}^{t}(1,1,1)>$ $P_{1}^{t}(1,0,0)$ for $M>M^{t}(1,0,0)$. 


\subsubsection{Step 2 For $M$ between $M^{t}(0,1,0)$ and $M^{t}(1,1,1), x=(1,1,1)$ is a CPNE and any CPNE generates the same outcome as $x=(1,1,1)$}

Proof. Step 1 implies that $x=(1,1,1)$ is a $\mathrm{NE}$ of $G^{m}$. In order to see that it is a CPNE, notice that (i) in $G_{1,2}^{m}(1), 1$ and 2 cannot both increase their profit, as they gain $M$ in aggregate with $\left(x_{1}, x_{2}\right)=(1,1)$; (ii) in $G_{1,3}^{m}(1)$, in order for 3 to make a profit it is necessary that $x_{1}=0$ and $M>M^{t}(0,0,1)$, and then 3 's unique best reply is $x_{3}=1$, so that 1's profit is $P_{1}^{t}(0,0,1)$ which is smaller than $P_{1}^{d}$; (iii) a similar argument applies to $G_{2,3}^{m}(1)$.

Finally, we prove that any CPNE is such that the active publishers and prices are as described in the statement of the proposition.

For $M \leq M^{t}(0,1,0)$, see our argument just before step 1 .

For $M^{t}(0,1,0)<M \leq M^{t}(0,0,1), 3$ is active only if $x=(1,0,1)$. But $x=(1,0,1)$ is not a NE of $G^{m}$, as 2 can induce 3 to be inactive by playing $x_{2}=1$, and this is profitable for 2 as $P_{2}^{d}>P_{2}^{t}(0,1,0)$. Hence, 3 is inactive in any NE and so in any CPNE.

For $M^{t}(0,0,1)<M \leq M^{t}(0,0,0), 3$ is active only if $x=(1,0,1)$ or $x=(0,1,1)$. We can show as above that $x=(1,0,1)$ is not a NE of $G^{m}$ because 2 prefers to play $x_{2}=1$, in order to induce 3 to be non-active. Furthermore, $P_{1}^{d}>P_{1}^{t}(0,0,1)$ and thus 1 prefers to play $x_{1}=1$ given $x_{2}=x_{3}=1$, so that 3 will not be active. Hence, $x=(0,1,1)$ is not a $\mathrm{NE}$ and any CPNE is such that 3 will be inactive.

For $M^{t}(0,0,0)<M \leq M^{t}(1,1,1)$, there are three profiles which allow 3 to be active: $x=(1,0,1), x=(0,1,1)$, and $x=(0,0,0)$, but none of them is a CPNE. The first two can be ruled out as above, while $x=(0,0,0)$ has been ruled out in Lemma 4 .

\subsubsection{Step 3 For $M$ between $M^{t}(1,1,1)$ and $U-I_{12}, x=(1,1,1)$ is the unique CPNE}

Proof. Step 1 implies that $x=(1,1,1)$ is a $\mathrm{NE}$ of $G^{m}$, and in order to see that it is a CPNE we notice that when $M>M^{t}(1,1,1)$, for each publisher $i$ playing $x_{i}=1$ strictly dominates $x_{i}=0$ if at least one of the other publishers interconnects: formally, if $x_{j}=1$ and/or $x_{k}=1$ then $x_{i}=1$ strictly dominates $x_{i}=0$ for publisher $i$. This implies that in $G_{i, j}^{m}(1)$, the only NE is $\left(x_{i}, x_{j}\right)=(1,1)$ and thus $x=(1,1,1)$ is a CPNE.

In order to prove uniqueness, recall that Lemma 4 shows that $x=(0,0,0)$ is not a CPNE. This implies that $x_{i}=1$ for at least one publisher $i$ in any CPNE. But then $x_{j}=1$ $\left(x_{k}=1\right)$ strictly dominates $x_{j}=0\left(x_{k}=0\right)$ for publisher $j$ (for publisher $k$ ) and this rules out any $x \neq(1,1,1)$. 


\subsection{Proof of Proposition 2 when A1 does not hold but A1 ${ }^{w}$ holds}

By backward induction, we first study the game $\gamma_{I I}$ for a given interconnection profile, in the order of $y=(0,1,0), y=(0,0,1), y=(1,0,0), y=(1,1,1)$. And then, we analyze the stage one interconnection game. A $1^{w}$ is used only when we study the case of $y=(1,1,1)$.

\subsubsection{The case of $y=(0,1,0)$}

Consider $y=(0,1,0)$. Then, $U_{1} \geq U_{2}+I_{23}$ in A1 does not play any role since $y_{23}=0$ and $U_{1}>U_{2}$. Hence, in order to relax A1, we suppose $U_{3}+I_{13}>U_{2}$. Step 1 in the proof of Lemma 1 implies that 1 is always active, given that $y_{23}=0$. However, Step 2 in the proof of Lemma 1 does not apply since $U_{3}+I_{13}>U_{2}$ and therefore $A=\{1\}$, or $A=\{1,2\}$, or $A=\{1,3\}$, or $A=\{1,2,3\}$. In particular, $P_{2}^{t}(0,1,0)=U_{2}+\frac{1}{3}\left(M-U-2 I_{13}\right)<$ $P_{3}^{t}(0,1,0)=U_{3}+\frac{1}{3}\left(M-U+I_{13}\right)$ and $P_{2}^{t}(0,1,0)>0$ if and only if $M>U-3 U_{2}+2 I_{13}$. Next lemma characterizes the cases in which $A=\{1,2\}$ or $A=\{1,3\}$.

Lemma 7 Suppose that $y=(0,1,0)$ and $U_{3}+I_{13}>U_{2}$. Then

(i) there exists a SPNE of $\gamma_{I I}$ in which $A=\{1,2\}$ if and only if $U_{1}-U_{2}<M \leq U_{1}-U_{3}$;

(ii) there exists a SPNE of $\gamma_{I I}$ in which $A=\{1,3\}$ if and only if $U_{1}-U_{3}<M \leq$ $U-3 U_{2}+2 I_{13}$.

Proof. (i) It is clear why $U_{1}-U_{2}<M$ is needed. About $M \leq U_{1}-U_{3}$, notice that if 3 deviates by becoming active, then it is necessary that in $\gamma_{I I I}$ a $\mathrm{NE}\left(P_{1}^{*}, P_{2}^{*}, P_{3}^{*}\right)$ is played such that 3 makes no profit. We show that the existence of such a NE requires $M \leq U_{1}-U_{3}$.

(a) Both 1 and 2 make a profit with $\left(P_{1}^{*}, P_{2}^{*}, P_{3}^{*}\right) \quad$ Then $u(12) \geq \max \{u(1), u(2)\}$. But if 3 plays $P_{3}>0$ close to zero, then $u(13) \simeq U_{1}+U_{3}+I_{13}-P_{1}^{*}>u(12)=$ $U_{1}+U_{2}-P_{1}^{*}-P_{2}^{*}$; therefore the library buys either $\mathrm{B}_{1} \& \mathrm{~B}_{3}$, or $\mathrm{B}_{2} \& \mathrm{~B}_{3}$, or $\mathrm{B}_{3}$ and 3 earns $P_{3}>0$.

(b) Only 1 makes a profit with $\left(P_{1}^{*}, P_{2}^{*}, P_{3}^{*}\right)$ Then $P_{1}^{*}=M$ and we need to consider various cases.

In the case of $P_{2}^{*}=0$ (independently of whether $P_{3}^{*}=0$ or $P_{3}^{*}>0$ ), we can show that if $M>U_{1}-U_{3}$, then 3 can earn a profit by playing $P_{3}>0$ close to zero. In that case, $\mathrm{B}_{1} \& \mathrm{~B}_{3}$ is not affordable and we have: $u(23)>\max \{u(2), u(3)\}, u(12)>u(1)$ and $u(23) \simeq U_{2}+U_{3}$ is larger than $u(12)=U_{1}+U_{2}-M$. 
In the case of $P_{2}^{*}>0, P_{3}^{*}>0$, the library buys only $\mathrm{B}_{1}$ but 2 may deviate with $P_{2}>0$ close to zero: then $\mathrm{B}_{1} \& \mathrm{~B}_{2}$ and $\mathrm{B}_{1} \& \mathrm{~B}_{3}$ are unaffordable and $u(2)>\max \{u(1), u(3)\}$ given $M>U_{1}-U_{3}>U_{1}-U_{2}$. This contradicts that only 1 makes a profit.

In the case of $P_{2}^{*}>0, P_{3}^{*}=0$, the library buys $\mathrm{B}_{1} \& \mathrm{~B}_{3}$ but 3 may deviate with $P_{3}>0$ close to zero. Then we need to distinguish three cases: $P_{2}^{*}<M, P_{2}^{*}=M, P_{2}^{*}>M$. In the first case $\mathrm{B}_{1} \& \mathrm{~B}_{2}$ and $\mathrm{B}_{1} \& \mathrm{~B}_{3}$ are unaffordable, $u(23)>u(2)$ and $u(3) \simeq U_{3}$ is larger than $u(1)=U_{1}-M$ if $M>U_{1}-U_{3}$. In the second case $\mathrm{B}_{1} \& \mathrm{~B}_{2}, \mathrm{~B}_{1} \& \mathrm{~B}_{3}, \mathrm{~B}_{2} \& \mathrm{~B}_{3}$ are unaffordable and $u(3)$ is larger than $u(1)=U_{1}-M>u(2)=U_{2}-M$ if $M>U_{1}-U_{3}$. In the third case $\mathrm{B}_{2}$ and $\mathrm{B}_{1} \& \mathrm{~B}_{3}$ are unaffordable and $u(3)>u(1)$ if $M>U_{1}-U_{3}$.

(c) Only 2 makes a profit with $\left(P_{1}^{*}, P_{2}^{*}, P_{3}^{*}\right)$ Then $P_{2}^{*}=M$ and it is easy to rule out the case (c) since we can show that 1 can find a profitable deviation. We need to consider two cases.

In the case of $P_{3}^{*}=0$ (independently of whether $P_{1}^{*}=0$ or $P_{1}^{*}>0$ ), 1 can make a profit by playing $P_{1}>0$ close to zero since then $\mathrm{B}_{1} \& \mathrm{~B}_{2}$ is unaffordable and $u(13) \simeq U_{1}+U_{3}+I_{13}>$ $\max \{u(1), u(2), u(3), u(23)\}$.

In the case of $P_{3}^{*}>0$ (independently of whether $P_{1}^{*}=0$ or $P_{1}^{*}>0$ ), 1 can make a profit by playing $P_{1}>0$ close to zero since then $\mathrm{B}_{1} \& \mathrm{~B}_{2}$ and $\mathrm{B}_{2} \& \mathrm{~B}_{3}$ are unaffordable and $u(1)>\max \{u(2), u(3)\}$.

We conclude from the previous arguments (a)-(c) that if $M>U_{1}-U_{3}$, then there exists no NE of $\gamma_{I I I}$ in which 3 earns zero.

We now show that if instead $M \leq U_{1}-U_{3}$, then

$$
P_{1}^{*}=M, \quad P_{2}^{*}=0, \quad P_{3}^{*}=0
$$

is a NE of $\gamma_{I I I}$. In order to see this, notice that if 2 deviates with $P_{2}>0$ close to zero, then $\mathrm{B}_{1} \& \mathrm{~B}_{2}$ is unaffordable, $u(23)>u(2)$ and $u(13)=U_{1}+U_{3}+I_{13}-M, u(23) \simeq U_{2}+U_{3}$. The inequality $u(13)>u(23)$ holds since $M \leq U_{1}-U_{3}$ and thus the library buys $\mathrm{B}_{1} \& \mathrm{~B}_{3}$ after 2's deviation. If 3 deviates with $P_{3}>0$ close to zero, then $\mathrm{B}_{1} \& \mathrm{~B}_{3}$ is unaffordable, $u(23)>u(3)$ and $u(12)=U_{1}+U_{2}-M, u(23) \simeq U_{2}+U_{3}$. The inequality $u(12)>u(23)$ holds because $M \leq U_{1}-U_{3}$.

(ii) It is clear why $U_{1}-U_{3}<M$ is needed. About $M \leq U-3 U_{2}+2 I_{13}$, notice that if 2 deviates by becoming active, then it is necessary that in $\gamma_{I I I}$ a $\mathrm{NE}\left(P_{1}^{*}, P_{2}^{*}, P_{3}^{*}\right)$ is played such that 2 makes no profit. Step 2 in the proof of Lemma 1 shows that we need to consider two cases, given that 1 makes a profit in any NE.

(a) Both 1 and 3 make a profit with $\left(P_{1}^{*}, P_{2}^{*}, P_{3}^{*}\right)$ No profitable deviation for 2 should exist, and thus it is necessary that $P_{2}>0$ close to zero does not induce the library 
to buy $\mathrm{B}_{1} \& \mathrm{~B}_{2}$ or $\mathrm{B}_{2} \& \mathrm{~B}_{3}$. Hence, the following conditions need to hold

$$
\begin{aligned}
& U_{1}+U_{2}-P_{1}^{*} \leq U_{1}+U_{3}+I_{13}-M \\
& U_{2}+U_{3}-P_{3}^{*} \leq U_{1}+U_{3}+I_{13}-M
\end{aligned}
$$

By adding up these inequalities we find $M \leq U-3 U_{2}+2 I_{13}$. For instance

$$
P_{1}^{*}=\frac{1}{2}\left(U_{1}+M-U_{3}\right), \quad P_{2}^{*} \text { high }, \quad P_{3}^{*}=\frac{1}{2}\left(U_{3}+M-U_{1}\right)
$$

is a NE of $\gamma_{I I I}$ for any $M$ between $U_{1}-U_{3}$ and $M \leq U-3 U_{2}+2 I_{13}$. Precisely, if 2 deviates with $P_{2}$ close to zero then $u(13)=U_{1}+U_{3}+I_{13}-M>u(23) \simeq U_{2}+U_{3}-\frac{1}{2}\left(U_{3}+M-U_{1}\right)$ and $u(13)=U_{1}+U_{3}+I_{13}-M>u(12)=U_{1}+U_{2}-\frac{1}{2}\left(U_{1}+M-U_{3}\right)$ are both satisfied since $M \leq U-3 U_{2}+2 I_{13}$.

(b) Only 1 makes a profit with $\left(P_{1}^{*}, P_{2}^{*}, P_{3}^{*}\right)$ This requires $P_{1}^{*}=M, P_{3}^{*}=0$. A deviation of 2 with $P_{2}>0$ close to zero is unprofitable if and only if $u(23) \simeq U_{2}+$ $U_{3} \leq u(13)=U_{1}+U_{3}+I_{13}-M$, which is equivalent to $M<U_{1}+I_{13}-U_{2}$. Since $U_{1}+I_{13}-U_{2}<U-3 U_{2}+2 I_{13}$, we conclude that this case is more restrictive than the first case, and thus we neglect it.

By relying on Lemma 7, we find the active publishers and prices as a function of $M$, as described by Lemma 8.

Lemma 8 Suppose that $y=(0,1,0)$ and $U_{3}+I_{13}>U_{2}$. Then any SPNE of $\gamma_{I I}$ is such that

(i) $A^{*}=\{1\}$ and $P_{1}^{*}=M$ if $M \leq U_{1}-U_{2}$;

(ii) $A^{*}=\{1,2\}$ and prices are $P_{1}^{d}, P_{2}^{d}$ if $U_{1}-U_{2}<M \leq U_{1}-U_{3}$;

(iii) $A^{*}=\{1,3\}$ and prices are $P_{1,3}^{d}=\frac{1}{2}\left(U_{1}+M-U_{3}\right), P_{3,1}^{d}=\frac{1}{2}\left(U_{3}+M-U_{1}\right)$ if $U_{1}-U_{3}<M \leq U-3 U_{2}+2 I_{13}$;

(iv) $A^{*}=\{1,2,3\}$ and prices are $P_{1}^{t}(0,1,0), P_{2}^{t}(0,1,0), P_{3}^{t}(0,1,0)$ if $U-3 U_{2}+2 I_{13}<$ $M<U-I_{12}$.

Proof. (i) It is impossible that $A^{*}=\{1,2\}$ since 1 and 2 cannot both make a profit if they are active and $M \leq U_{1}-U_{2}$. The same argument applies to rule out $A^{*}=\{1,3\}$ and $A^{*}=\{1,2,3\}$.

(ii) It is impossible that $A^{*}=\{1\}$ because then 2 would deviate by becoming active, and both $A^{*}=\{1,3\}$ and $A^{*}=\{1,2,3\}$ are ruled out since $M \leq U_{1}-U_{3}$. In particular, given $A^{*}=\{1,2\}$, if 3 becomes active then the NE described in (24) is played.

(iii) We argue like in part (ii) to rule out $A^{*}=\{1\}$. It is impossible that $A^{*}=\{1,2\}$ because then 3 would deviate by becoming active. If 2 becomes active, then the NE described in (25) is played.

(iv) If $A=\{1\}$, or $A=\{1,2\}$, or $A=\{1,3\}$, then at least a publisher who is inactive deviates by becoming active. 


\subsubsection{The case of $y=(0,0,1)$}

Consider $y=(0,0,1)$. Then, $U_{2} \geq U_{3}+I_{13}$ in A1 does not play any role since $y_{13}=0$ and $U_{2}>U_{3}$. Hence, in order to relax A1, we suppose that $U_{2}+I_{23}>U_{1}$. We need to distinguish two cases:

$$
\begin{aligned}
U_{2}+I_{23}>U_{1}>U_{3}+I_{23} \\
U_{3}+I_{23}>U_{1}
\end{aligned}
$$

In the first case $P_{2}^{t}(0,0,1)=U_{2}+\frac{1}{3}\left(M-U+I_{23}\right)>P_{1}^{t}(0,0,1)=U_{1}+\frac{1}{3}(M-U-$ $\left.2 I_{23}\right)>P_{3}^{t}(0,0,1)=U_{3}+\frac{1}{3}\left(M-U+I_{23}\right)$, thus all three publishers can be active only if $M>M^{t}(0,0,1)$. In the second case, instead, $P_{2}^{t}(0,0,1)>P_{3}^{t}(0,0,1)>P_{1}^{t}(0,0,1)$ holds and all three publishers can be active only if $M>U-3 U_{1}+2 I_{23}$. In the first case we obtain the same results as when A1 holds, as it is intuitive given that $P_{3}^{t}(0,0,1)$ is smaller than $P_{1}^{t}(0,0,1)$ and than $P_{2}^{t}(0,0,1)$.

Lemma 9 Suppose that $y=(0,0,1)$ and that (26) holds. Then Lemma 1 applies.

Proof. Using (26) we can rule out the case of $A=\{2,3\}$ as follows. Suppose that $A=\{2,3\}$ and 1 deviates by becoming active. From Step 1 in the proof of Lemma 1, we see that if, in this case, there exists a NE $\left(P_{1}^{*}, P_{2}^{*}, P_{3}^{*}\right)$ of $\gamma_{I I I}$ in which 1 makes no profit, then $P_{2}^{*}=M, P_{3}^{*}=0$. But the existence of such a NE requires that

$$
U_{1}+U_{3} \leq U_{2}+U_{3}+I_{23}-M
$$

Since $A=\{2,3\}$ requires $M>U_{2}-U_{3}$, it follows that (28) is violated. Therefore, 1 is always active when (26) holds.

Furthermore, we can also rule out the case of $A=\{1,3\}$ by arguing as in Step 2 of the proof of Lemma 1 , since $U_{2} \geq U_{3}+I_{13} y_{13}$ holds given that $y_{13}=0$.

Now we consider the case in which (27) is satisfied.

Lemma 10 Suppose that $y=(0,0,1)$ and (27) holds. Then

(i) there exists a SPNE of $\gamma_{I I}$ in which $A=\{1,2\}$ if and only if $U_{1}-U_{2}<M \leq$ $\min \left\{U_{1}-U_{3}, U_{2}+I_{23}-U_{1}\right\}$;

(ii) there exists a SPNE of $\gamma_{I I}$ in which $A=\{2,3\}$ if and only if $U_{2}-U_{3}<M \leq$ $U-3 U_{1}+2 I_{23}$.

Proof. (i) If is clear why $U_{1}-U_{2}<M$ is needed. About $M \leq \min \left\{U_{1}-U_{3}, U_{2}+I_{23}-U_{1}\right\}$, notice that if 3 deviates by becoming active, then it is necessary that in $\gamma_{I I I}$ a $\mathrm{NE}$ $\left(P_{1}^{*}, P_{2}^{*}, P_{3}^{*}\right)$ is played such that 3 makes no profit. We show below that the existence of such a NE requires $M \leq \min \left\{U_{1}-U_{3}, U_{2}+I_{23}-U_{1}\right\}$. We consider three cases. 
(a) Both 1 and 2 make a profit with $\left(P_{1}^{*}, P_{2}^{*}, P_{3}^{*}\right) \quad$ Then $u(12) \geq \max \{u(1), u(2)\}$ but 3 can deviate with $P_{3}>0$ close to zero. Then $u(23) \simeq U_{2}+U_{3}+I_{23}-P_{2}^{*}>u(12)=$ $U_{1}+U_{2}-P_{1}^{*}-P_{2}^{*}$; therefore the library buys either $\mathrm{B}_{1} \& \mathrm{~B}_{3}$, or $\mathrm{B}_{2} \& \mathrm{~B}_{3}$, or $\mathrm{B}_{3}$ and 3 earns $P_{3}>0$.

(b) Only 1 makes a profit with $\left(P_{1}^{*}, P_{2}^{*}, P_{3}^{*}\right)$ Then $P_{1}^{*}=M$ and we need to consider several cases.

In the case of $P_{3}^{*}=0$ (independently of whether $P_{2}^{*}>0$ or $\left.P_{2}^{*}=0\right), 2$ can make a profit by playing $P_{2}>0$ close to zero since then $\mathrm{B}_{1} \& \mathrm{~B}_{2}$ is unaffordable, $u(23)>\max \{u(2), u(3)\}$, $u(13)>u(1)$ and $u(23) \simeq U_{2}+U_{3}+I_{23}>u(13)=U_{1}+U_{3}-M$.

In the case of $P_{2}^{*}>0, P_{3}^{*}>0$, the library buys only $\mathrm{B}_{1}$ but 2 may deviate with $P_{2}>0$ close to zero: then $\mathrm{B}_{1} \& \mathrm{~B}_{2}$ and $\mathrm{B}_{1} \& \mathrm{~B}_{3}$ are unaffordable and $u(2)>\max \{u(1), u(3)\}$ since $M>U_{1}-U_{2}$.

In the case of $P_{2}^{*}=0, P_{3}^{*}>0$, the library buys $\mathrm{B}_{1} \& \mathrm{~B}_{2}$ so that $u(12) \geq \max \{u(1), u(2)\}$ but 3 may deviate with $P_{3}>0$ close to zero and then $\mathrm{B}_{1} \& \mathrm{~B}_{3}$ is unaffordable, $u(23)>$ $\max \{u(2), u(3)\}$ and $u(23) \simeq U_{2}+U_{3}+I_{23}>u(12)=U_{1}+U_{2}-M$.

(c) Only 2 makes a profit with $\left(P_{1}^{*}, P_{2}^{*}, P_{3}^{*}\right)$ Then $P_{2}^{*}=M$ and we need to consider several cases.

In the case of $P_{1}^{*}=0$ (independently of whether $P_{3}^{*}>0$ or $P_{3}^{*}=0$ ), consider 3 playing $P_{3}>0$ close to zero so that $\mathrm{B}_{2} \& \mathrm{~B}_{3}$ is unaffordable, $u(13)>\max \{u(1), u(3)\}, u(12)>u(2)$ and $u(13) \simeq U_{1}+U_{3}, u(12)=U_{1}+U_{2}-M$; the inequality $u(13)>u(12)$ holds if $M>U_{2}-U_{3}$. Since $U_{2}-U_{3}<\min \left\{U_{1}-U_{3}, U_{2}+I_{23}-U_{1}\right\}$, if $M>\min \left\{U_{1}-U_{3}, U_{2}+I_{23}-U_{1}\right\}$ then 3 's deviation is profitable.

In the case of $P_{1}^{*}>0, P_{3}^{*}>0$, the library buys only $\mathrm{B}_{2}$ but 1 may deviate with $P_{1}>0$ close to zero: then $\mathrm{B}_{1} \& \mathrm{~B}_{2}$ and $\mathrm{B}_{2} \& \mathrm{~B}_{3}$ are unaffordable and $u(1)>\max \{u(2), u(3)\}$.

In the case of $P_{1}^{*}>0, P_{3}^{*}=0$, we need to distinguish three cases: $P_{1}^{*}<M, P_{1}^{*}=M$, $P_{1}^{*}>M$. In the third case, if $M>U_{2}-U_{3}$ then 3 can make a profit with $P_{3}>0$ close to zero, since then $\mathrm{B}_{1}$ and $\mathrm{B}_{2} \& \mathrm{~B}_{3}$ are unaffordable and $u(3)>u(2)$. In the first case, 3 can make a profit with $P_{3}>0$ close to zero because then $\mathrm{B}_{1} \& \mathrm{~B}_{2}$ and $\mathrm{B}_{2} \& \mathrm{~B}_{3}$ are unaffordable and $u(13)>u(1), u(13)=U_{1}+U_{3}-P_{1}^{*}>u(2)=U_{2}-M$. In the second case, instead, we find that

$$
P_{1}^{*}=M, \quad P_{2}^{*}=M, \quad P_{3}^{*}=0
$$

is a $\mathrm{NE}$ if $M \leq \min \left\{U_{1}-U_{3}, U_{2}+I_{23}-U_{1}\right\}$, in which the library buys $\mathrm{B}_{2} \& \mathrm{~B}_{3}$ as $u(23)>$ $u(13)$. Precisely, if 1 deviates with $P_{1}>0$ close to zero then $\mathrm{B}_{1} \& \mathrm{~B}_{2}$ is unaffordable and $u(13)>\max \{u(1), u(3)\}, u(23)>u(2)$, and $u(13) \simeq U_{1}+U_{3}, u(23)=U_{2}+U_{3}+I_{23}-M$. The inequality $U_{1}+U_{3} \leq U_{2}+U_{3}+I_{23}-M$ is equivalent to $M \leq U_{2}+I_{23}-U_{1}$. If 3 deviates with $P_{3}>0$ close to zero then $\mathrm{B}_{1} \& \mathrm{~B}_{2}, \mathrm{~B}_{1} \& \mathrm{~B}_{3}$ and $\mathrm{B}_{2} \& \mathrm{~B}_{3}$ are unaffordable and 
$u(1)=U_{1}-M>u(2)$, while $u(3) \simeq U_{3}$. The inequality $U_{3} \leq U_{1}-M$ is equivalent to $M \leq U_{1}-U_{3}$.

Therefore, we conclude that there exists a NE of $\gamma_{I I I}$ in which 3 earns zero if and only if $M \leq \min \left\{U_{1}-U_{3}, U_{2}+I_{23}-U_{1}\right\}$.

(ii) It is clear why $M>U_{2}-U_{3}$ is needed. About $M \leq U-3 U_{1}+2 I_{23}$, notice that if 1 deviates by becoming active, then it is necessary that in $\gamma_{I I I}$ a $\mathrm{NE}\left(P_{1}^{*}, P_{2}^{*}, P_{3}^{*}\right)$ is played such that 1 makes no profit. Step 1 in the proof of Lemma 1 shows that we need to consider three cases.

(a) Both 2 and 3 make a profit with $\left(P_{1}^{*}, P_{2}^{*}, P_{3}^{*}\right) \quad$ No profitable deviation for 1 should exist, and thus it is necessary that $P_{1}>0$ close to zero does not induce the library to buy $\mathrm{B}_{1} \& \mathrm{~B}_{2}$ or $\mathrm{B}_{1} \& \mathrm{~B}_{3}$. Hence, the following conditions need to hold

$$
\begin{aligned}
& U_{1}+U_{2}-P_{2}^{*} \leq U_{2}+U_{3}+I_{23}-M \\
& U_{1}+U_{3}-P_{3}^{*} \leq U_{2}+U_{3}+I_{23}-M
\end{aligned}
$$

By adding up these inequalities we find $M \leq U-3 U_{1}+2 I_{23}$ and, for instance

$$
P_{1}^{*} \text { large, } \quad P_{2}^{*}=\frac{1}{2}\left(U_{2}+M-U_{3}\right), \quad P_{3}^{*}=\frac{1}{2}\left(U_{3}+M-U_{2}\right)
$$

is a NE of $\gamma_{I I I}$ for any $M$ such that $U_{2}-U_{3}<M \leq U-3 U_{1}+2 I_{23}$.

(b) Only 2 makes a profit with $\left(P_{1}^{*}, P_{2}^{*}, P_{3}^{*}\right)$ This requires $P_{2}^{*}=M, P_{3}^{*}=0$. A deviation of 1 with $P_{1}>0$ close to zero is unprofitable if and only if $u(13) \simeq U_{1}+$ $U_{3} \leq u(23)=U_{2}+U_{3}+I_{23}-M$, which is equivalent to $M<U_{2}+I_{23}-U_{1}$. Since $U_{2}+I_{23}-U_{1}<U-3 U_{1}+2 I_{23}$, we conclude that this case is more restrictive than the first case, and thus we neglect it.

(c) Only 3makes a profit with $\left(P_{1}^{*}, P_{2}^{*}, P_{3}^{*}\right)$ This requires $P_{2}^{*}=0, P_{3}^{*}=M$. A deviation of 1 with $P_{1}>0$ close to zero is unprofitable if and only if $u(12) \simeq U_{1}+$ $U_{2} \leq u(23)=U_{2}+U_{3}+I_{23}-M$, which is equivalent to $M<U_{3}+I_{23}-U_{1}$. Since $U_{3}+I_{23}-U_{1}<U-3 U_{1}+2 I_{23}$, we conclude that this case is more restrictive than the first case, and thus we neglect it.

By relying on Lemma 10 we find the active publishers and prices as a function of $M$, as described by the following lemma, which is divided in three parts because it is necessary to distinguish there cases according to the values of $U_{1}-U_{2}, U_{2}-U_{3}$, and $\min \left\{U_{1}-U_{3}, U_{2}+I_{23}-U_{1}\right\}$. In doing this, we need however to take into account that

$$
U_{2}-U_{3}<\min \left\{U_{1}-U_{3}, U_{2}+I_{23}-U_{1}\right\}<U-3 U_{1}+2 I_{23}
$$


Lemma 11 Suppose that $y=(0,0,1)$ and that (27) holds.

(i) When $U_{1}-U_{2} \leq U_{2}-U_{3}<\min \left\{U_{1}-U_{3}, U_{2}+I_{23}-U_{1}\right\}$, any SPNE of $\gamma_{I I}$ is such that (ia) $A^{*}=\{1\}$ and $P_{1}^{*}=M$ if $M<U_{1}-U_{2}$;

(ib) $A^{*}=\{1,2\}$ and $P_{1,2}^{d}, P_{2,1}^{d}$ if $U_{1}-U_{2}<M \leq U_{2}-U_{3}$;

(ic) $A^{*}=\{1,2\}$ and $P_{1,2}^{d}, P_{2,1}^{d}$ or $A^{*}=\{2,3\}$ and $P_{2,3}^{d}, P_{3,2}^{d}$ if $U_{2}-U_{3}<M \leq$ $\min \left\{U_{1}-U_{3}, U_{2}+I_{23}-U_{1}\right\}$

(id) $A^{*}=\{2,3\}$ and $P_{2,3}^{d}, P_{3,2}^{d}$ if $\min \left\{U_{1}-U_{3}, U_{2}+I_{23}-U_{1}\right\}<M \leq U-3 U_{1}+2 I_{23}$;

(ie) $A^{*}=\{1,2,3\}$ and $P_{1}^{t}(0,0,1), P_{2}^{t}(0,0,1), P_{3}^{t}(0,0,1)$ if $U-3 U_{1}+2 I_{23}<M<U-I_{12}$. (ii) When $U_{2}-U_{3}<U_{1}-U_{2} \leq \min \left\{U_{1}-U_{3}, U_{2}+I_{23}-U_{1}\right\}$, any SPNE of $\gamma_{I I}$ is such that

(iia) $A^{*}=\{1\}$ and $P_{1}^{*}=M$ if $M \leq U_{2}-U_{3}$;

(iib) $A^{*}=\{1\}$ and $P_{1}^{*}=M$ or $A^{*}=\{2,3\}$ and $P_{2,3}^{d}, P_{3,2}^{d}$ if $U_{2}-U_{3}<M \leq U_{1}-U_{2}$;

(iic) $A^{*}=\{1,2\}$ and $P_{1,2}^{d}, P_{2,1}^{d}$ or $A^{*}=\{2,3\}$ and $P_{2,3}^{d}, P_{3,2}^{d}$ if $U_{1}-U_{2}<M \leq$ $\min \left\{U_{1}-U_{3}, U_{2}+I_{23}-U_{1}\right\}$;

(iid) $A^{*}=\{2,3\}$ and $P_{2,3}^{d}, P_{3,2}^{d}$ if $\min \left\{U_{1}-U_{3}, U_{2}+I_{23}-U_{1}\right\}<M \leq U-3 U_{1}+2 I_{23}$;

(iie) $A^{*}=\{1,2,3\}$ and $P_{1}^{t}(0,0,1), P_{2}^{t}(0,0,1), P_{3}^{t}(0,0,1)$ if $U-3 U_{1}+2 I_{23}<M<$ $U-I_{12}$.

(iii) When $U_{2}-U_{3}<\min \left\{U_{1}-U_{3}, U_{2}+I_{23}-U_{1}\right\}<U_{1}-U_{2}$, any SPNE of $\gamma_{I I}$ is such that

(iiia) $A^{*}=\{1\}$ and $P_{1}^{*}=M$ if $M \leq U_{2}-U_{3}$;

(iiib) $A^{*}=\{1\}$ and $P_{1}^{*}=M$ or $A^{*}=\{2,3\}$ and $P_{2,3}^{d}, P_{3,2}^{d}$ if $U_{2}-U_{3}<M \leq U_{1}-U_{2}$;

(iiic) $A^{*}=\{2,3\}$ and $P_{2,3}^{d}, P_{3,2}^{d}$ if $U_{1}-U_{2}<M \leq U-3 U_{1}+2 I_{23}$;

(iiid) $A^{*}=\{1,2,3\}$ and $P_{1}^{t}(0,0,1), P_{2}^{t}(0,0,1), P_{3}^{t}(0,0,1)$ if $U-3 U_{1}+2 I_{23}<M \leq$ $U-I_{12} \cdot{ }^{47}$

Proof. (ia) Given that $M<U_{1}-U_{2}$, and moreover $M<U_{2}-U_{3}, M<U-3 U_{1}+2 I_{23}$, it is impossible that $A^{*}=\{1,2\}$, or $A^{*}=\{2,3\}$, or $A^{*}=\{1,2,3\}$ because there is no NE of $\gamma_{I I I}$ in which at least two publishers make a profit. Hence $A^{*}=\{1\}$.

(ib) For $U_{1}-U_{2}<M \leq U_{2}-U_{3}, A^{*}=\{1,2\}$, because 2 becomes active if $A=\{1\}$. But there is no NE in $\gamma_{I I I}$ in which both 2 and 3 make a profit if $A=\{2,3\}$, and there is no NE in which $1,2,3$ make a profit if $A=\{1,2,3\}$. If 3 becomes active given $A^{*}=\{1,2\}$, then the NE described in (29) is played.

(ic) For $U_{2}-U_{3}<M \leq \min \left\{U_{1}-U_{3}, U_{2}+I_{23}-U_{1}\right\}, A^{*} \neq\{1\}$; otherwise 2 becomes active. There is no NE in $\gamma_{I I I}$ in which $1,2,3$ make a profit if $A=\{1,2,3\}$. There is a SPNE in $\gamma_{I I}$ with $A^{*}=\{1,2\}$ just like in part (ib). But there is also a SPNE with $A^{*}=\{2,3\}$ and then, if 1 becomes active, the NE described in (30) is played.

\footnotetext{
${ }^{47}$ Say that $U-3 U_{1}+2 I_{23}<U_{1}-U_{2}$. Then $U_{2}-U_{3}<M \leq U-3 U_{1}+2 I_{23}$ implies $A^{*}=\{1\}$ or $A^{*}=\{2,3\} ; U-3 U_{1}+2 I_{23}<M<U_{1}-U_{2}$ implies $A^{*}=\{1\}$ or $A^{*}=\{1,2,3\} ; U_{1}-U_{2}<M$ implies $A^{*}=\{1,2,3\}$.
} 
(id) For $\min \left\{U_{1}-U_{3}, U_{2}+I_{23}-U_{1}\right\}<M<U-3 U_{1}+2 I_{23}$, we find that $A^{*}=\{2,3\}$ because 2 becomes active if $A=\{1\}, 3$ becomes active if $A=\{1,2\}$, and there is no NE of $\gamma_{I I I}$ in which all three publishers make a profit if $A=\{1,2,3\}$. If 1 enters, then the NE described in (30) is played.

(ie) For $U-3 U_{1}+2 I_{23}<M<U-I_{12}$, if $A$ does not include the three publishers then some publisher who is inactive has an incentive to deviate by becoming active, given that he will be able to make a profit; thus $A^{*}=\{1,2,3\}$

In order to prove (iia)-(iie) and (iiia)-(iiid) it suffices to apply suitably the same arguments given in the proof of (ia)-(ie).

\subsubsection{The cases of $y=(1,0,0)$ or $y=(1,1,1)$}

When $y=(1,0,0)$ or $y=(1,1,1)$, then the inequalities $U_{1}+I_{12} y_{12}>U_{3}+I_{23} y_{23}$, $U_{1}+I_{13} y_{13} \geq U_{2}+I_{23} y_{23}, U_{1}+I_{12} y_{12}>U_{3}+I_{23} y_{23}, U_{2}+I_{12} y_{12} \geq U_{3}+I_{13} y_{13}$ and $U_{2}+I_{23} y_{23} \geq U_{3}+I_{13} y_{13}$ are all satisfied if $\mathrm{A} 1$ fails to hold, provided that $\mathrm{A} 1^{w}$ is satisfied. In this case, therefore, Lemma 3 holds.

\subsubsection{The interconnection stage}

Since $U_{3}+I_{23}>U_{1}$ implies $U_{3}+I_{13}>U_{2}$, a necessary condition to obtain results different from Lemma 1 is $U_{3}+I_{13}>U_{2}$. Therefore, we need to study the following cases: (i) $U_{3}+I_{13}>U_{2}$ and $U_{3}+I_{23} \leq U_{1}$; (ii) $U_{3}+I_{23}>U_{1}$. We consider below only the second case, as the first one is similar to (but simpler than) the second.

Step 1 For each publisher $i$, playing $x_{i}=1$ weakly dominates $x_{i}=0 \quad$ Proof. In this case Lemma 1 applies if $y=(0,0,0), y=(1,0,0), y=(1,1,1)$, but Lemma 8 applies if $y=(0,1,0)$ and Lemma 10 applies if $y=(0,0,1)$. Nevertheless, we can prove weakly dominance 


\begin{tabular}{|c|c|}
\hline $\begin{array}{c}1 \\
x_{1}=0 \\
x_{1}=1\end{array}$ & $\begin{array}{c}x_{2}=1, x_{3}=0 \\
M \text { if } M \leq U_{1}-U_{2}, P_{1,2}^{d} \text { if } U_{1}-U_{2}<M \leq M^{t}(0,0,0), P_{1}^{t}(0,0,0) \text { if } M>M^{t}(0,0,0) \\
M \text { if } M \leq U_{1}-U_{2}, P_{1,2}^{d} \text { if } U_{1}-U_{2}<M \leq M^{t}(1,0,0) \\
P_{1}^{t}(1,0,0) \text { if } M>M^{t}(1,0,0)\end{array}$ \\
\hline $\begin{array}{c}1 \\
x_{1}=0 \\
x_{1}=1\end{array}$ & $\begin{array}{c}x_{2}=0, x_{3}=1 \\
M \text { if } M \leq U_{1}-U_{2}, P_{1,2}^{d} \text { if } U_{1}-U_{2}<M \leq M^{t}(0,0,0), P_{1}^{t}(0,0,0) \text { if } M>M^{t}(0,0,0) \\
M \text { if } M \leq U_{1}-U_{2}, P_{1,2}^{d} \text { if } U_{1}-U_{2}<M \leq U_{1}-U_{3}, \\
P_{1,3}^{d} \text { if } U_{1}-U_{3}<M \leq U-3 U_{2}+2 I_{13}, P_{1}^{t}(0,1,0) \text { if } M>U-3 U_{2}+2 I_{13}\end{array}$ \\
\hline $\begin{aligned} & 1 \\
x_{1} & =0 \\
x_{1} & =1\end{aligned}$ & $\begin{array}{c}x_{2}=1, x_{3}=1 \\
M\left(\text { requires } M \leq U_{1}-U_{2}\right) \text { or } P_{1,2}^{d}\left(\text { requires } M \leq \min \left\{U_{1}-U_{3}, U_{2}+I_{23}-U_{1}\right\}\right) \\
\text { or } 0 \text { or } P_{1}^{t}(0,0,1) \\
M \text { if } M \leq U_{1}-U_{2}, P_{1,2}^{d} \text { if } U_{1}-U_{2}<M \leq M^{t}(1,1,1), P_{1}^{t}(1,1,1) \text { if } M>M^{t}(1,1,1)\end{array}$ \\
\hline
\end{tabular}

In order to see that $x_{1}=1$ weakly dominates $x_{1}=0$ for 1 , notice that $M^{t}(0,0,0)<$ $M^{t}(1,0,0)$ and $P_{1,2}^{d}>P_{1}^{t}(0,0,0)$ for $M$ between $M^{t}(0,0,0)$ and $M^{t}(1,0,0), P_{1}^{t}(1,0,0)>$ $P_{1}^{t}(0,0,0)$ for $M>M^{t}(1,0,0) ; U_{1}-U_{3}<M^{t}(0,0,0)$ and $P_{1,3}^{d}>P_{1,2}^{d}, P_{1,3}^{d}>P_{1}^{t}(0,0,0)$, $P_{1}^{t}(0,1,0)>P_{1,2}^{d}$ for $M \leq M^{t}(0,0,0), P_{1}^{t}(0,1,0)>P_{1}^{t}(0,0,0)$ for $M>M^{t}(0,0,0)$; $P_{1,2}^{d}>P_{1}^{t}(0,0,1), P_{1}^{t}(1,1,1)>P_{1,2}^{d}$ for $M \leq U_{1}-U_{3}, P_{1}^{t}(1,1,1)>P_{1}^{t}(0,0,1)$.

\begin{tabular}{|c|c|}
\hline $\begin{aligned} 2 & \\
x_{2} & =0 \\
x_{2} & =1\end{aligned}$ & $\begin{array}{c}\quad x_{1}=1, x_{3}=0 \\
0 \text { if } M \leq U_{1}-U_{2}, P_{2,1}^{d} \text { if } U_{1}-U_{2}<M \leq M^{t}(0,0,0), P_{2}^{t}(0,0,0) \text { if } M>M^{t}(0,0,0) \\
0 \text { if } M \leq U_{1}-U_{2}, P_{2,1}^{d} \text { if } U_{1}-U_{2}<M \leq M^{t}(1,0,0), P_{2}^{t}(1,0,0) \text { if } M>M^{t}(1,0,0)\end{array}$ \\
\hline $\begin{aligned} 2 & \\
x_{2} & =0 \\
x_{2} & =1\end{aligned}$ & $\begin{array}{c}x_{1}=0, x_{3}=1 \\
0 \text { if } M \leq U_{1}-U_{2}, P_{2,1}^{d} \text { if } U_{1}-U_{2}<M \leq M^{t}(0,0,0), P_{2}^{t}(0,0,0) \text { if } M>M^{t}(0,0,0) \\
\left.0 \text { (requires } M \leq U_{1}-U_{2}\right) \text { or } P_{2,1}^{d}\left(\text { requires } M \leq \min \left\{U_{1}-U_{3}, U_{2}+I_{23}-U_{1}\right\}\right) \\
\text { or } P_{2,3}^{d} \text { or } P_{2}^{t}(0,0,1)\end{array}$ \\
\hline $\begin{aligned} & 2 \\
x_{2} & =0 \\
x_{2} & =1\end{aligned}$ & $\begin{array}{c}x_{1}=1, x_{3}=1 \\
0 \text { if } M \leq U_{1}-U_{2}, P_{2,1}^{d} \text { if } U_{1}-U_{2}<M \leq U_{1}-U_{3} \\
0 \text { if } U_{1}-U_{3}<M \leq U-3 U_{2}+2 I_{13}, P_{2}^{t}(0,1,0) \text { if } M>U-3 U_{2}+2 I_{13} \\
0 \text { if } M \leq U_{1}-U_{2}, P_{2,1}^{d} \text { if } U_{1}-U_{2}<M \leq M^{t}(1,1,1), P_{2}^{t}(1,1,1) \text { if } M>M^{t}(1,1,1)\end{array}$ \\
\hline
\end{tabular}

In order to see that $x_{2}=1$ weakly dominates $x_{2}=0$ for 2 , notice that $M^{t}(0,0,0)<$ $M^{t}(1,0,0)$ and $P_{2,1}^{d}>P_{2}^{t}(0,0,0)$ for $M$ between $M^{t}(0,0,0)$ and $M^{t}(1,0,0), P_{2}^{t}(1,0,0)>$ $P_{2}^{t}(0,0,0)$ for $M>M^{t}(1,0,0) ; P_{2,3}^{d}>P_{2,1}^{d}, P_{2,3}^{d}>P_{2}^{t}(0,0,0), P_{2}^{t}(0,0,1)>P_{2,1}^{d}, P_{2}^{t}(0,0,1)>$ $P_{2}^{t}(0,0,0) ; U_{1}-U_{3}<M^{t}(1,1,1)$ and $P_{2,1}^{d}>0, P_{2,1}^{d}>P_{2}^{t}(0,1,0), P_{2}^{t}(1,1,1)>P_{2}^{t}(0,1,0)$.

\begin{tabular}{|cc}
\hline 3 & $x_{1}=1, x_{2}=0$ \\
$x_{3}=0$ & 0 if $M \leq M^{t}(0,0,0), P_{3}^{t}(0,0,0)$ if $M>M^{t}(0,0,0)$ \\
$x_{3}=1$ & 0 if $M \leq U_{1}-U_{3}, P_{3,1}^{d}$ if $U_{1}-U_{3}<M \leq U-3 U_{2}+2 I_{13}, P_{3}^{t}(0,1,0)$ if $M>U-3 U_{2}+2 I_{13}$ \\
\hline
\end{tabular}




\begin{tabular}{|cc|}
\hline $\begin{array}{c}3 \\
x_{3}=0\end{array}$ & $x_{1}=0, x_{2}=1$ \\
$x_{3}=1$ & 0 (requires $\left.M \leq \min \left\{U_{1}-U_{3}, U_{2}+I_{23}-U_{1}\right\}\right)$ or $P_{3,2}^{d}$ or $P_{3}^{t}(0,0,1)$ \\
\cline { 2 - 2 } & $x_{1}=1, x_{2}=1$ \\
$x_{3}=0$ & 0 if $M \leq M^{t}(1,0,0), P_{3}^{t}(1,0,0)$ if $M>M^{t}(1,0,0)$ \\
$x_{3}=1$ & 0 if $M \leq M^{t}(1,1,1), P_{3}^{t}(1,1,1)$ if $M>M^{t}(1,1,1)$
\end{tabular}

In order to see that $x_{3}=1$ weakly dominates $x_{3}=0$ for 3 , notice that $U_{1}-U_{3}<$ $M^{t}(0,0,0)$ and $P_{3,1}^{d}>P_{3}^{t}(0,0,0), P_{3}^{t}(0,1,0)>P_{3}^{t}(0,1,0) ; \min \left\{U_{1}-U_{3}, U_{2}+I_{23}-U_{1}\right\}<$ $M^{t}(0,0,0)$ and $P_{3,2}^{d}>P_{3}^{t}(0,0,0), P_{3}^{t}(0,0,1)>P_{3}^{t}(0,0,0) ; M^{t}(1,1,1)<M^{t}(1,0,0)$ and $P_{3}^{t}(1,1,1)>0$ for $M$ between $M^{t}(1,1,1)$ and $M^{t}(1,0,0), P_{3}^{t}(1,1,1)>P_{1}^{t}(1,0,0)$ for $M>M^{t}(1,1,1)$.

Step 2 For $M$ between $U_{1}-U_{2}<M \leq M^{t}(1,1,1), x=(1,1,1)$ is a CPNE and any CPNE generates the same outcome Proof. Step 1 implies that $x=(1,1,1)$ is a NE. In order to verify that $x=(1,1,1)$ is a CPNE we notice that in $G_{1,2}^{m}(1)$ it is impossible to improve for both 1 and 2 . In $G_{1,3}^{m}(1), 3$ may gain a positive profit only if $x_{1}=0$, but then 1 earns $P_{1}^{d}$, or $P_{1}^{t}(0,0,0)$, or 0 , or $P_{1}^{t}(0,0,1)$. Since none of them is larger than $P_{1}^{d}$, it is impossible that 1 and 3 both improve in $G_{1,3}^{m}(1)$. In $G_{2,3}^{m}(1), 3$ may gain a positive profit only if $x_{2}=0$, but then 2 earns $P_{2}^{d}$ or $P_{2}^{t}(0,0,0)$ or 0 or $P_{2}^{t}(0,1,0)$. Since none of them is larger than $P_{2}^{d}$, it is impossible that 2 and 3 both improve in $G_{2,3}^{m}(1)$.

Furthermore, we prove that any CPNE generates the same outcome as $x=(1,1,1)$. A different outcome requires that 3 is active, and for this to occur it is necessary that $y=(0,1,0)$, or $y=(0,0,1)$, or $y=(0,0,0)$. We can exclude $y=(0,0,0)$ since Lemma 4 applies also when A1 is violated and establishes that $x=(0,0,0)$ is not a CPNE. We can exclude $y=(0,1,0)$ since if 3 is active, then 2 earns either 0 or $P_{2}^{t}(0,1,0)$, but 2 can earn $P_{2}^{d}$ by playing $x_{2}=1$; since $P_{2}^{d}>\max \left\{0, P_{2}^{t}(0,1,0)\right\}$, there is no NE with $y=(0,1,0)$ in which 3 is active. A very similar argument applies to rule out $y=(0,0,1)$ : if 3 is active, then 1 earns either 0 or $P_{1}^{t}(0,0,1)$, but he can earn $P_{1}^{d}$ by playing $x_{1}=1$; since $P_{1}^{d}>\max \left\{0, P_{1}^{t}(0,0,1)\right\}$, there is no NE with $y=(0,0,1)$ in which 3 is active.

Step 3 For $M$ smaller than $U_{1}-U_{2}, x=(1,1,1)$ is a CPNE and any CPNE generates the same outcome Proof. When $M \leq U_{1}-U_{2}$, we find that $A^{*}=\{1\}$ for any $y$ except $y=(0,0,1)$, in which case $A^{*}=\{2,3\}$ and 1 earns zero. But 1 can be sure to earn $M$ by playing $x_{1}=1$, hence any NE (and CPNE) is such that $A^{*}=\{1\}$. It is routine to verify that $x=(1,1,1)$ is a CPNE.

Step 4 For $M$ between $M^{t}(1,1,1)$ and $U-I_{12}, x=(1,1,1)$ is the unique CPNE Proof. Step 3 in the proof of Proposition 2 applies since, even though A1 is violated 
(i) it is still true that $x=(1,1,1)$ is a $\mathrm{NE}$ of $G^{m}$ (see step 1 in this proof); (ii) given $M>M^{t}(1,1,1)$, for each publisher $i$ playing $x_{i}=1$ strictly dominates $x_{i}=0$ if at least one of the other publishers interconnects; (iii) Lemma 4 establishes that $x=(0,0,0)$ is not a CPNE.

\subsection{Proof of Lemma 5}

We argue by contradiction and suppose that $y_{13}^{*}=0$ (the case of $y_{23}^{*}=0$ can be dealt with almost exactly in the same way, and thus is omitted). This requires that $x_{13}^{*}=0$, since 3 would play $x_{31}=1$ if $x_{13}^{*}$ were equal to 1 and $y_{13}=1$ would result; for a similar reason, $x_{31}^{*}=0$. The proof relies on the fact that in $G_{1,3}^{b}\left(\mathbf{x}_{2}^{*}\right)$ there exists a NE $\left(\mathbf{x}_{1}^{\prime}, \mathbf{x}_{3}^{\prime}\right)$ such that $\mathbf{x}_{1}^{\prime}=\left(x_{12}^{*}, 1\right)$ and $\mathbf{x}_{3}^{\prime}=(1,1)$. The resulting interconnection profile $y^{\prime}=\left(y_{12}^{\prime}, y_{13}^{\prime}, y_{23}^{\prime}\right)$ is such that $y_{12}^{\prime}=y_{12}^{*}$ and $y_{13}^{\prime}=1$. About $y_{23}^{\prime}$, if $x_{23}^{*}=0$ then $y_{23}^{\prime}=0=y_{23}^{*}$; if $x_{23}^{*}=1$, then $x_{32}^{*}=1$ since $\mathbf{x}^{*}$ is a NE and $\mathbf{x}_{3}^{*}$ is a best reply to $\mathbf{x}_{1}^{*}, \mathbf{x}_{2}^{*}$, thus $y_{23}^{*}=1=y_{23}^{\prime}$. Hence, the only difference between $y^{\prime}$ and $y^{*}$ is $y_{13}^{\prime}=1 \neq y_{13}^{*}=0$, and the profit of both 1 and 3 with $\left(\mathbf{x}_{1}^{\prime}, \mathbf{x}_{3}^{\prime}\right)$ is higher by $\frac{1}{3} I_{13}$ than with $\left(\mathbf{x}_{1}^{*}, \mathbf{x}_{3}^{*}\right)$. This establishes that $\mathbf{x}^{*}$ is not a CPNE if we prove that $\left(\mathbf{x}_{1}^{\prime}, \mathbf{x}_{3}^{\prime}\right)$ is a $\mathrm{NE}$ of $G_{1,3}^{b}\left(\mathbf{x}_{2}^{*}\right)$.

In order to prove that $\mathbf{x}_{3}^{\prime}$ is a best reply to $\mathbf{x}_{1}^{\prime}$, we notice that $\mathbf{x}_{3}=(1,1)$ is a weakly dominant strategy for publisher 3 because it weakly reduces $M^{t}(y)$ and weakly increases 3 's profit, $P_{3}^{t}(y)$, if 3 is active.

In order to prove that $\mathbf{x}_{1}^{\prime}$ is a best reply to $\mathbf{x}_{3}^{\prime}$, we first notice that against $\mathbf{x}_{3}^{\prime}, \mathbf{x}_{1}^{\prime}$ is better than $\mathbf{x}_{1}^{*}=\left(x_{12}^{*}, 0\right)$ because it yields a profit higher by $\frac{1}{3} I_{13}$ (recall that 3 is active if 1 plays $\left.\mathbf{x}_{1}^{*}\right)$. We now show that $\mathbf{x}_{1}^{\prime}$ is a (weakly) better reply also than $\left(\tilde{x}_{12}^{*}, 0\right)$ and $\left(\tilde{x}_{12}^{*}, 1\right)$, where $\tilde{x}_{12}^{*}$ is the opposite choice with respect to $x_{12}^{*}$, that is $\tilde{x}_{12}^{*}=1-x_{12}^{*}$. There are three cases to consider: (i) if $x_{21}^{*}=0$, then $\tilde{x}_{12}^{*}$ has no effect; (ii) if $x_{21}^{*}=1$ and $x_{12}^{*}=1$, then playing $\tilde{x}_{12}^{*}=0$ yields 1 a profit which is by $\frac{1}{3} I_{12}$ smaller than his profit with $x_{12}^{*}=1$ (recall that 3 is active if 1 plays $\mathbf{x}_{1}^{*}$ ); (iii) if $x_{21}^{*}=1$ and $x_{12}^{*}=0$, then we obtain a contradiction as follows. Since $x_{12}^{*}=0$, we infer that 1 prefers the interconnection profile $y^{*}=\left(0,0, y_{23}^{*}\right)$ to $y=\left(1,0, y_{23}^{*}\right)$; notice that $M>M^{t}\left(0,0, y_{23}^{*}\right)$ in order for 3 to be active. With the profile $y^{*}$, 1 earns $P_{1}^{t}\left(0,0, y_{23}^{*}\right)$ and with $y=\left(1,0, y_{23}^{*}\right)$ he would earn either $P_{1}^{d}$ if $M \leq M^{t}\left(1,0, y_{23}^{*}\right)$ or $P_{1}^{t}\left(1,0, y_{23}^{*}\right)$ if $M>M^{t}\left(1,0, y_{23}^{*}\right)$. But $P_{1}^{d}>P_{1}^{t}\left(0,0, y_{23}^{*}\right)$ for $M$ between $M^{t}\left(0,0, y_{23}^{*}\right)$ and $M^{t}\left(1,0, y_{23}^{*}\right)$, and $P_{1}^{t}\left(1,0, y_{23}^{*}\right)>P_{1}^{t}\left(0,0, y_{23}^{*}\right)$ for $M>M^{t}\left(1,0, y_{23}^{*}\right)$. Hence, $x_{21}^{*}=1$ and $x_{12}^{*}=0$ cannot occur in a NE.

\subsection{Proof of Proposition 3}

In the strong version of proposition 3, (iii) and (iv) are stated as follows 
(iii) for $M$ between $M^{t}(1,1,1)$ and $M^{t}(1,1,0): A^{*}=\{1,2\}$ if

$$
P_{1}^{d} \geq P_{1}^{t}(0,1,0) \quad \text { or } \quad\left(P_{1}^{t}(0,1,0)>P_{1}^{d} \geq P_{1}^{t}(1,1,1) \quad \text { and } \quad P_{2}^{d} \geq P_{2}^{t}(0,0,1)\right)
$$

but $A^{*}=\{1,2,3\}$ with $y=(0,1,1)$ otherwise;

(iv) for $M$ between $M^{t}(1,1,0)$ and $M^{t}(1,0,1)$ : $A^{*}=\{1,2\}$ if

$$
P_{1}^{d} \geq P_{1}^{t}(1,1,1) \quad \text { and } \quad P_{2}^{d} \geq P_{2}^{t}(0,0,1)
$$

but $A^{*}=\{1,2,3\}$ with $y=(0,1,1)$ if $P_{1}^{d}>P_{1}^{t}(1,1,1)$ and $P_{2}^{d}<P_{2}^{t}(0,0,1)$, and $A^{*}=$ $\{1,2,3\}$ with $y=(1,1,1)$ if $P_{1}^{t}(1,1,1) \geq P_{1}^{d}$;

The strong version includes the weak version. In what follows, we prove the strong version. Note that we do not need to prove the results for $U_{1}-U_{2}<M \leq M^{t}(0,1,0)$ and $M^{t}(1,0,0)<M \leq U-I_{12}$ since they are explained in the main text just before the statement of the proposition.

Proof for the case of $M^{t}(0,1,0)<M \leq M^{t}(1,1,1)$

Step 1 If $P_{1}^{t}(0,1,1)>P_{1}^{d}$, then 3 is active and $y=(0,1,1)$ in any CPNE.

Proof. Suppose that $\mathbf{x}^{*}$ is such that 3 is non-active; then we show that $\mathbf{x}^{*}$ is not a CPNE: then, 3 is active in any CPNE and $M \leq M^{t}(1,1,1)$ and Lemma 5 imply that $y=(0,1,1)$ in any CPNE.

The profits of 1 and 3 with $\mathbf{x}^{*}$ are $P_{1}^{d}$ and zero, respectively, and now we prove that $\mathbf{x}_{1}^{\prime}=(0,1), \mathbf{x}_{3}^{\prime}=(1,1)$ is a NE of $G_{1,3}^{b}\left(\mathbf{x}_{2}^{*}\right)$ which both 1 and 3 prefer to $\left(\mathbf{x}_{1}^{*}, \mathbf{x}_{3}^{*}\right)$. About $\mathbf{x}_{3}^{\prime}$, we know that it is a best reply to $\mathbf{x}_{1}^{\prime}$. About $\mathbf{x}_{1}^{\prime}$, notice that it generates $y=\left(0,1, x_{23}^{*}\right)$ and then 1 's profit is $P_{1}^{t}\left(0,1, x_{23}^{*}\right)$, while $\mathbf{x}_{1}=(0,0)$ generates $y=\left(0,0, x_{23}^{*}\right)$ and then 1 's profit is either $P_{1}^{d}$ or $P_{1}^{t}\left(0,0, x_{23}^{*}\right)$, depending on whether $M \leq M^{t}\left(0,0, x_{23}^{*}\right)$ or $M>$ $M^{t}\left(0,0, x_{23}^{*}\right)$; since $P_{1}^{t}\left(0,1, x_{23}^{*}\right) \geq P_{1}^{t}(0,1,1)>P_{1}^{d}$ and $P_{1}^{t}\left(0,1, x_{23}^{*}\right)>P_{1}^{t}\left(0,0, x_{23}^{*}\right)$, in either case 1 prefers $\mathbf{x}_{1}^{\prime}$ to $\mathbf{x}_{1}=(0,0)$. Regarding $\mathbf{x}_{1}=(1,1)$ and $\mathbf{x}_{1}=(1,0)$, they generate $y=\left(x_{21}^{*}, 1, x_{23}^{*}\right)$ and $y=\left(x_{21}^{*}, 0, x_{23}^{*}\right)$, respectively. If $x_{21}^{*}=0$, there is no difference with respect to the comparison between $\mathbf{x}_{1}^{\prime}$ and $\mathbf{x}_{1}=(0,0)$. If $x_{21}^{*}=1$, then 3 is excluded and 1 gains $P_{1}^{d}$. But since $P_{1}^{t}\left(0,1, x_{23}^{*}\right) \geq P_{1}^{t}(0,1,1)>P_{1}^{d}$ holds, we infer that $\mathbf{x}_{1}^{\prime}$ is 1 's unique best reply against $\mathbf{x}_{3}^{\prime}$. Thus $\left(\mathbf{x}_{1}^{\prime}, \mathbf{x}_{3}^{\prime}\right)$ is a NE of $G_{1,3}^{b}\left(\mathbf{x}_{2}^{*}\right)$ which Pareto dominates $\left(\mathbf{x}_{1}^{*}, \mathbf{x}_{3}^{*}\right)$, as the profits of 1 and 3 are $P_{1}^{t}\left(0,1, x_{23}^{*}\right)>P_{1}^{d}$ and $P_{3}^{t}\left(0,1, x_{23}^{*}\right)>0$ respectively. As a consequence, $\mathbf{x}^{*}$ is not a CPNE and 3 is active in any CPNE.

Step 2 If $P_{1}^{t}(0,1,1)>P_{1}^{d}$, then (for instance) $\mathbf{x}_{1}^{*}=\mathbf{x}_{2}^{*}=(0,1), \mathbf{x}_{3}^{*}=(1,1)$ is a CPNE. Proof. In order to verify that $\mathbf{x}^{*}$ is a NE, it suffices to notice that (i) 1 could exclude 3 (for some values of $M$ ), but $P_{1}^{t}(0,1,1)>P_{1}^{d}$ and thus he prefers to play $x_{13}=1$; (ii) 2 cannot exclude 3 , given $\mathbf{x}_{1}^{*}$ and $\mathbf{x}_{3}^{*}$, and so maximizes the own profit by playing $x_{23}=1$; (iii) 3 is playing the own weakly dominant strategy.

In order to verify that $\mathbf{x}^{*}$ is a CPNE, we examine $G_{1,2}^{b}(1,1), G_{1,3}^{b}(0,1), G_{2,3}^{b}(0,1)$. 
- In $G_{1,2}^{b}(1,1)$, publisher 1 cannot make a profit higher than $P_{1}^{t}(0,1,1)$ if 3 is nonactive, as $P_{1}^{d}<P_{1}^{t}(0,1,1)$. Thus $y_{12}=0$, and the profit of 1 may be higher than $P_{1}^{t}(0,1,1)$ only if $y_{23}=0$. But this cannot occur since 2 plays $x_{23}=1$ in any NE, as $x_{32}^{*}=1$.

- In $G_{1,3}^{b}(0,1), 3$ cannot earn more than $P_{3}^{t}(0,1,1)$, as that is his highest feasible profit. $^{48}$

- In $G_{2,3}^{b}(0,1), 3$ cannot earn more than $P_{3}^{t}(0,1,1)$, as that is his highest feasible profit.

Step 3 If $P_{1}^{d}>P_{1}^{t}(0,1,1)$, then 3 is non-active in any CPNE.

Proof. Suppose that $\mathbf{x}^{*}$ is a CPNE such that 3 is active; then Lemma 5 implies $y^{*}=$ $(0,1,1)$ and hence the profits of 1 and 2 are $P_{1}^{t}(0,1,1)$ and $P_{2}^{t}(0,1,1)$, respectively. We now prove that $\mathbf{x}_{1}^{\prime}=\mathbf{x}_{2}^{\prime}=(1,1)$ is a $\mathrm{NE}$ in $G_{1,2}^{b}(1,1)$ which both 1 and 2 prefer to $\left(\mathbf{x}_{1}^{*}, \mathbf{x}_{2}^{*}\right)$. When 2 plays $\mathbf{x}_{2}^{\prime}$, 1's profit from playing $\mathbf{x}_{1}^{\prime}$ or $\mathbf{x}_{1}=(1,0)$ is $P_{1}^{d}$; if instead 1 plays $\mathbf{x}_{1}=(0,1)$ or $\mathbf{x}_{1}=(0,0)$, then he earns $P_{1}^{t}(0,1,1)$, or $P_{1}^{t}(0,0,1)$, or $P_{1}^{d}$. The assumption $P_{1}^{d}>P_{1}^{t}(0,1,1)$ guarantees that $\mathbf{x}_{1}^{\prime}$ is a best reply to $\mathbf{x}_{2}^{\prime}$, and a very similar argument reveals that $\mathbf{x}_{2}^{\prime}$ is a best reply to $\mathbf{x}_{1}^{\prime}$. Thus $\left(\mathbf{x}_{1}^{\prime}, \mathbf{x}_{2}^{\prime}\right)$ is a NE of $G_{1,2}^{b}(1,1)$ which Pareto dominates $\left(\mathbf{x}_{1}^{*}, \mathbf{x}_{2}^{*}\right): 3$ is non-active in this NE and the profits of 1 and 2 are $P_{1}^{d}>P_{1}^{t}(0,1,1)$ and $P_{2}^{d}>P_{2}^{t}(0,1,1)$ respectively. As a consequence, $\mathbf{x}^{*}$ is not a CPNE and there exists no CPNE such that 3 is active.

Step 4 If $P_{1}^{d}>P_{1}^{t}(0,1,1)$, then (for instance) $\mathbf{x}_{1}^{*}=\mathbf{x}_{2}^{*}=(1,1), \mathbf{x}_{3}^{*}=(0,0)$ is a CPNE. Proof. When $M \leq M^{t}(0,0,0)$, it is clear that $\mathbf{x}^{*}$ is a NE, since no firm can individually modify the fact that 3 is not going to be active. For $M>M^{t}(0,0,0), \mathbf{x}^{*}$ is a NE since $P_{1}^{d}>P_{1}^{t}(0,0,0)$ and $P_{2}^{d}>P_{2}^{t}(0,0,0)$ hold.

In order to verify that $\mathbf{x}^{*}$ is a CPNE, we examine $G_{1,2}^{b}(0,0), G_{1,3}^{b}(1,1), G_{2,3}^{b}(1,1)$.

- In $G_{1,2}^{b}\left(\mathbf{x}_{3}^{*}\right), 1$ and 2 cannot both increase their profit, as they gain $M$ in aggregate with $\left(\mathbf{x}_{1}^{*}, \mathbf{x}_{2}^{*}\right) \cdot{ }^{49}$

- In $G_{1,3}^{b}(1,1)$, if 3 is active, then $x_{12}=0$ must hold. Furthermore, $x_{32}=1$ in any NE as $x_{23}^{*}=1$. Thus $y_{23}=1$ and the highest profit 1 can make is $P_{1}^{t}(0,1,1)$, which is smaller than $P_{1}^{d}$ by assumption.

- In $G_{2,3}^{b}(1,1)$, if 3 is active, then $x_{21}=0$ must hold. Furthermore, $x_{31}=1$ in any NE as $x_{13}^{*}=1$. Thus $y_{13}=1$ and the highest profit 2 can make is $P_{2}^{t}(0,1,1)$, which is smaller than $P_{2}^{d}$.

\footnotetext{
${ }^{48}$ As a general result, if $\mathbf{x}^{*}$ is such that $y=(0,1,1)$, then in $G_{1,3}^{b}\left(\mathbf{x}_{2}^{*}\right)$ and $G_{2,3}^{b}\left(\mathbf{x}_{1}^{*}\right)$ publisher 3 cannot earn more than $P_{3}^{t}(0,1,1)$, as that is his highest feasible profit.

${ }^{49}$ As a general result, if $\mathbf{x}^{*}$ is such that 3 is non-active then 1 and 2 cannot both increase their profits in $G_{1,2}^{b}\left(\mathbf{x}_{3}^{*}\right)$, as they gain $M$ in aggregate with $\left(\mathbf{x}_{1}^{*}, \mathbf{x}_{2}^{*}\right)$.
} 
The proof for the case of $M^{t}(1,1,1)<M \leq M^{t}(1,1,0)$

Step 1 There exists a CPNE such that 3 is inactive if and only if

$$
P_{1}^{d} \geq P_{1}^{t}(0,1,0) \quad \text { or } \quad\left(P_{1}^{d} \geq P_{1}^{t}(1,1,1) \text { and } P_{2}^{d} \geq P_{2}^{t}(0,0,1)\right)
$$

Proof. Since $M^{t}(1,1,1)<M \leq M^{t}(1,1,0)$, if a CPNE $\mathbf{x}^{*}$ is such that 3 is inactive, then $y_{12}^{*}=1$ and $x_{13}^{*}=0$ and/or $x_{23}^{*}=0$, otherwise $M>M^{t}(y)$ holds if 3 plays $\mathbf{x}_{3}=(1,1)$. In terms of $\left(\mathbf{x}_{1}^{*}, \mathbf{x}_{2}^{*}\right)$, this is equivalent to $\left(\mathbf{x}_{1}^{*}=(1,1), \mathbf{x}_{2}^{*}=(1,0)\right)$, or $\left(\mathbf{x}_{1}^{*}=(1,0), \mathbf{x}_{2}^{*}=(1,1)\right)$, or $\left(\mathbf{x}_{1}^{*}=(1,0), \mathbf{x}_{2}^{*}=(1,0)\right)$.

- Step 1.1 There exists a CPNE such that 3 is inactive and $\mathbf{x}_{1}^{*}=(1,1), \mathbf{x}_{2}^{*}=(1,0)$ if and only if $P_{1}^{d} \geq P_{1}^{t}(0,1,0)$.

About $G_{1,2}^{b}\left(\mathbf{x}_{3}^{*}\right)$, see footnote 49 .

Proof. In $G_{1,3}^{b}(1,0), 3$ is active if and only if $x_{12}=0$. In the case of $P_{1}^{t}(0,1,0)>P_{1}^{d}$, then $\mathbf{x}_{1}^{\prime}=(0,1), \mathbf{x}_{3}^{\prime}=(1,0)$ is a NE which Pareto dominates $\left(\mathbf{x}_{1}^{*}, \mathbf{x}_{3}^{*}\right)$ and this eliminates the candidate CPNE. If instead $P_{1}^{t}(0,1,0) \leq P_{1}^{d}$, then there exists no NE of $G_{1,3}^{b}(1,0)$ in which 1 earns a profit higher than $P_{1}^{d}$.

In $G_{2,3}^{b}(1,1), 3$ is active if and only if $\mathbf{x}_{2}=\mathbf{x}_{3}=(1,1)$ or $x_{21}=0$. In either case the profit of 2 cannot be higher than $P_{2}^{d}$. First, $P_{2}^{d}>P_{2}^{t}(1,1,1)$ holds, which rules out the first case. For the second case, given that $x_{13}^{*}=1,3$ plays $x_{31}=1$ in any NE and thus $y=(0,1,1)$ or $y=(0,1,0)$; but in each case 2 earns less than $P_{2}^{d}$.

Therefore, we conclude that there exists no CPNE such that 3 is inactive and $\mathbf{x}_{1}^{*}=$ $(1,1), \mathbf{x}_{2}^{*}=(1,0)$ if $P_{1}^{d}<P_{1}^{t}(0,1,0)$. If $P_{1}^{d} \geq P_{1}^{t}(0,1,0)$, the profile $\mathbf{x}_{1}^{*}=(1,1), \mathbf{x}_{2}^{*}=(1,0)$, $\mathbf{x}_{3}^{*}=(0,0)$ (for instance) is a NE and a CPNE.

- Step 1.2 There exists a CPNE such that 3 is inactive and $\mathbf{x}_{1}^{*}=(1,0), \mathbf{x}_{2}^{*}=(1,1)$ if and only if $P_{1}^{d} \geq P_{1}^{t}(1,1,1)$ and $P_{2}^{d} \geq P_{2}^{t}(0,0,1)$. Then the profile $\mathbf{x}_{1}^{*}=(1,0)$, $\mathbf{x}_{2}^{*}=(1,1), \mathbf{x}_{3}^{*}=(0,0)$ (for instance) is a CPNE.

Proof. About $G_{1,2}^{b}\left(\mathbf{x}_{3}^{*}\right)$, see footnote 49 .

In $G_{1,3}^{b}(1,1), 3$ is active if and only if $\mathbf{x}_{1}=\mathbf{x}_{3}=(1,1)$ or $x_{12}=0$. In particular, $\mathbf{x}_{1}^{\prime}=\mathbf{x}_{3}^{\prime}=(1,1)$ is a NE which Pareto dominates $\left(\mathbf{x}_{1}^{*}, \mathbf{x}_{3}^{*}\right)$ if and only if $P_{1}^{t}(1,1,1)>P_{1}^{d}$. If instead $P_{1}^{d} \geq P_{1}^{t}(1,1,1)$, then there is no NE of $G_{1,3}^{b}(1,1)$ in which 1 earns more than $P_{1}^{d}$. If $x_{12}=0$, then $P_{1}^{t}(0,1,1)$ is the highest feasible equilibrium profit for 1 (since 3 plays $x_{32}=1$ in any NE given that $\left.x_{23}^{*}=1\right)$ but $P_{1}^{t}(1,1,1)>P_{1}^{t}(0,1,1)$. Therefore, if $P_{1}^{d} \geq P_{1}^{t}(1,1,1)$, there exists no NE of $G_{1,3}^{b}(1,1)$ in which 1 earns a profit higher than $P_{1}^{d}$.

In $G_{2,3}^{b}(1,0), 3$ is active if and only if $x_{21}=0$. In the case of $P_{2}^{t}(0,0,1)>P_{2}^{d}$, then $\mathbf{x}_{2}^{\prime}=(0,1), \mathbf{x}_{3}^{\prime}=(0,1)$ is a NE which Pareto dominates $\left(\mathbf{x}_{2}^{*}, \mathbf{x}_{3}^{*}\right)$ and this eliminates the 
candidate CPNE. If instead $P_{2}^{d} \geq P_{2}^{t}(0,0,1)$, then there exists no NE of $G_{2,3}^{b}(1,0)$ in which 2 earns a profit higher than $P_{2}^{d}$.

Therefore, we conclude that there exists no CPNE such that 3 is inactive and $\mathbf{x}_{1}^{*}=$ $(1,0), \mathbf{x}_{2}^{*}=(1,1)$ if $P_{1}^{d}<P_{1}^{t}(1,1,1)$ or $P_{2}^{d}<P_{2}^{t}(0,0,1)$. If $P_{1}^{d} \geq P_{1}^{t}(1,1,1)$ and $P_{2}^{d} \geq$ $P_{2}^{t}(0,0,1)$, then the profile $\mathbf{x}_{1}^{*}=(1,0), \mathbf{x}_{2}^{*}=(1,1), \mathbf{x}_{3}^{*}=(0,0)$ (for instance) is a NE and a CPNE in which 3 is non-active.

- Step 1.3 If there exists a CPNE such that 3 is inactive and $\mathbf{x}_{1}^{*}=\mathbf{x}_{2}^{*}=(1,0)$, then also $\mathbf{x}_{1}^{*}=(1,1), \mathbf{x}_{2}^{*}=(1,0), \mathbf{x}_{3}^{*}=(0,0)$ is a CPNE with the same outcome.

Proof. Consider the candidate equilibrium in which 3 is inactive and $\mathbf{x}_{1}^{*}=\mathbf{x}_{2}^{*}=(1,0)$. In $G_{1,3}^{b}(1,0), 3$ is active if and only if $x_{12}=0$. If $P_{1}^{t}(0,1,0)>P_{1}^{d}$, then $\mathbf{x}_{1}^{\prime}=(0,1)$, $\mathbf{x}_{3}^{\prime}=(1,0)$ is a NE which Pareto dominates $\left(\mathbf{x}_{1}^{*}, \mathbf{x}_{3}^{*}\right)$ and this eliminates the candidate CPNE. Hence, $P_{1}^{d} \geq P_{1}^{t}(0,1,0)$ is a necessary condition to have such a CPNE. But, from step 1.1, this condition is sufficient for the existence of a CPNE with the same outcome, for instance $\mathbf{x}_{1}^{*}=(1,1), \mathbf{x}_{2}^{*}=(1,0), \mathbf{x}_{3}^{*}=(0,0)$.

Step 2 There exists a CPNE such that 3 is active if and only if (33) is violated. In such a case, the unique CPNE is $\mathbf{x}_{1}^{*}=\mathbf{x}_{2}^{*}=(0,1), \mathbf{x}_{3}^{*}=(1,1)$ and thus $y^{*}=(0,1,1)$.

Proof. If 3 is active, then $y=(0,1,1)$ or $y=(1,1,1)$ in any CPNE. However, it is simple to see that $\mathbf{x}_{1}=\mathbf{x}_{2}=\mathbf{x}_{3}=(1,1)$ is not a NE of $G^{b}$ because 2 can exclude 3 by playing $\mathbf{x}_{2}=(1,0)$ and $P_{2}^{d}>P_{2}^{t}(1,1,1)$. Thus, $\mathbf{x}_{2}=(1,0)$ is 2 's best reply given $\mathbf{x}_{1}=\mathbf{x}_{3}=(1,1)$.

In order to verify that $\mathbf{x}^{*}$ is a CPNE if and only if (33) does not hold, we examine $G_{1,2}^{b}(1,1), G_{1,3}^{b}(0,1), G_{2,3}^{b}(0,1)$.

- About $G_{1,3}^{b}\left(\mathbf{x}_{2}^{*}\right)$ and $G_{2,3}^{b}\left(\mathbf{x}_{1}^{*}\right)$, see footnote 48 .

- About $G_{1,2}^{b}(1,1)$, it is useful to see the normal form of this game:

\begin{tabular}{c|c|c|c|c|}
$\mathbf{x}_{1} \backslash \mathbf{x}_{2}$ & $\mathbf{x}_{2}=(1,0)$ & $\mathbf{x}_{2}=(0,1)$ & $\mathbf{x}_{2}=(1,1)$ & $\mathbf{x}_{2}=(0,0)$ \\
\cline { 2 - 5 }$(1,0)$ & $P_{1}^{d}, P_{2}^{d}$ & $P_{1}^{t}(0,0,1), P_{2}^{t}(0,0,1)$ & $P_{1}^{d}, P_{2}^{d}$ & $P_{1}^{t}(0,0,0), P_{2}^{t}(0,0,0)$ \\
\cline { 2 - 5 }$(0,1)$ & $P_{1}^{t}(0,1,0), P_{2}^{t}(0,1,0)$ & $P_{1}^{t}(0,1,1), P_{2}^{t}(0,1,1)$ & $P_{1}^{t}(0,1,1), P_{2}^{t}(0,1,1)$ & $P_{1}^{t}(0,1,0), P_{2}^{t}(0,1,0)$ \\
\cline { 2 - 5 }$(1,1)$ & $P_{1}^{d}, P_{2}^{d}$ & $P_{1}^{t}(0,1,1), P_{2}^{t}(0,1,1)$ & $P_{1}^{t}(1,1,1), P_{2}^{t}(1,1,1)$ & $P_{1}^{t}(0,1,0), P_{2}^{t}(0,1,0)$ \\
\cline { 2 - 5 }$(0,0)$ & $P_{1}^{t}(0,0,0), P_{2}^{t}(0,0,0)$ & $P_{1}^{t}(0,0,1), P_{2}^{t}(0,0,1)$ & $P_{1}^{t}(0,0,1), P_{2}^{t}(0,0,1)$ & $P_{1}^{t}(0,0,0), P_{2}^{t}(0,0,0)$ \\
\cline { 2 - 5 } & & &
\end{tabular}

- The only $\left(\mathbf{x}_{1}, \mathbf{x}_{2}\right)$ in which both 1 and 2 earn more than $P_{1}^{t}(0,1,1), P_{2}^{t}(0,1,1)$ are $\mathbf{x}_{1}=\mathbf{x}_{2}=(1,1)$, and the $\left(\mathbf{x}_{1}, \mathbf{x}_{2}\right)$ which lead to the exclusion of 3 . However, $\mathbf{x}_{1}=\mathbf{x}_{2}=(1,1)$ is not a NE since $P_{2}^{d}>P_{2}^{t}(1,1,1)$. On the other hand, $\mathbf{x}_{1}^{\prime}=(1,1)$, $\mathbf{x}_{2}^{\prime}=(1,0)$ is a NE which Pareto dominates $\mathbf{x}_{1}^{*}=\mathbf{x}_{2}^{*}=(0,1)$ if $P_{1}^{d} \geq P_{1}^{t}(0,1,0)$, while 
$\mathbf{x}_{1}^{\prime}=(1,0), \mathbf{x}_{2}^{\prime}=(1,1)$ is a NE which Pareto dominates $\left(\mathbf{x}_{1}^{*}, \mathbf{x}_{2}^{*}\right)$ if $P_{1}^{d} \geq P_{1}^{t}(1,1,1)$ and $P_{2}^{d} \geq P_{2}^{t}(0,0,1)$.

Therefore, $\mathbf{x}^{*}$ is a CPNE if and only if (33) is not satisfied. In order to see that $\mathbf{x}^{*}$ is the unique CPNE in this case, notice that (i) we have proved that $y^{*}=(0,1,1)$, thus it is necessary that $\mathbf{x}_{3}=(1,1)$ and $x_{13}=x_{23}=1$; (ii) if $x_{12}=1$, then 2 has an incentive to play $\mathbf{x}_{2}=(1,0)$ (which induces 3's exclusion), as noted above; (iii) if $x_{21}=1$, then 1 has an incentive to play $\mathbf{x}_{1}=(1,1)$ or $\mathbf{x}_{1}=(1,0)$, which implies a $y$ different from $(0,1,1)$.

The proof for the case of $M^{t}(1,1,0)<M \leq M^{t}(1,0,1)$

Step 1 A CPNE such that 3 is non-active exists if and only if

$$
P_{1}^{d} \geq P_{1}^{t}(1,1,1) \quad \text { and } \quad P_{2}^{d} \geq P_{2}^{t}(0,0,1)
$$

Proof. Suppose that a CPNE $\mathbf{x}^{*}$ is such that 3 is inactive; then $y_{12}^{*}=1$ and $x_{13}^{*}=0$, otherwise $M>M^{t}(y)$ holds if 3 plays $\mathbf{x}_{3}=(1,1)$; thus, either $\mathbf{x}_{1}^{*}=\mathbf{x}_{2}^{*}=(1,0)$ or $\mathbf{x}_{1}^{*}=(1,0), \mathbf{x}_{2}^{*}=(1,1)$.

In the case of $\mathbf{x}_{1}^{*}=\mathbf{x}_{2}^{*}=(1,0)$, we find that $\mathbf{x}_{1}^{\prime}=\mathbf{x}_{3}^{\prime}=(1,1)$ is a NE in $G_{1,3}^{b}(1,0)$ which both 1 and 3 prefer to $\left(\mathbf{x}_{1}^{*}, \mathbf{x}_{3}^{*}\right)$. Indeed, given $\mathbf{x}_{3}^{\prime}, 1$ can earn $P_{1}^{t}(0,0,0)$, or $P_{1}^{t}(0,1,0)$, or $P_{1}^{d}$, or $P_{1}^{t}(1,1,0)$, and $P_{1}^{t}(1,1,0)>P_{1}^{d}$ holds, which implies that $\mathbf{x}_{1}^{\prime}$ is 1 's unique best reply to $\mathbf{x}_{3}^{\prime}$. The NE $\left(\mathbf{x}_{1}^{\prime}, \mathbf{x}_{3}^{\prime}\right)$ Pareto dominates $\left(\mathbf{x}_{1}^{*}, \mathbf{x}_{3}^{*}\right)$, as the profits of 1 and 3 are $P_{1}^{t}(1,1,0)>P_{1}^{d}$ and $P_{3}^{t}(1,1,0)>0$ respectively. As a consequence, there is no CPNE $\mathbf{x}^{*}$ such that 3 is inactive and $\mathbf{x}_{2}^{*}=(1,0)$.

In the case of $\mathbf{x}_{1}^{*}=(1,0)$ and $\mathbf{x}_{2}^{*}=(1,1)$, we examine $G_{1,3}^{b}(1,1)$ and $G_{2,3}^{b}(1,0)$, as footnote 49 applies to $G_{1,2}^{b}\left(\mathbf{x}_{3}^{*}\right)$.

- In $G_{1,3}^{b}(1,1)$, if $P_{1}^{t}(1,1,1)>P_{1}^{d}$ then $\mathbf{x}_{1}^{\prime}=\mathbf{x}_{3}^{\prime}=(1,1)$ is a NE which 1 and 3 prefer to $\left(\mathbf{x}_{1}^{*}, \mathbf{x}_{3}^{*}\right)$. Indeed, given $\mathbf{x}_{3}^{\prime}, 1$ can earn $P_{1}^{t}(0,0,1)$, or $P_{1}^{t}(0,1,1)$, or $P_{1}^{d}$, or $P_{1}^{t}(1,1,1)$, and if $P_{1}^{t}(1,1,1)>P_{1}^{d}$ then $\mathbf{x}_{1}^{\prime}$ is a best reply to $\mathbf{x}_{3}^{\prime}$. Thus $\left(\mathbf{x}_{1}^{\prime}, \mathbf{x}_{3}^{\prime}\right)$ is a NE of $G_{1,3}^{b}\left(\mathbf{x}_{2}^{*}\right)$ which Pareto dominates $\left(\mathbf{x}_{1}^{*}, \mathbf{x}_{3}^{*}\right)$, as the profits of 1 and 3 are $P_{1}^{t}(1,1,1)>P_{1}^{d}$ and $P_{3}^{t}(1,1,1)>0$ respectively. As a consequence, $\mathbf{x}^{*}$ is not a CPNE. If instead $P_{1}^{t}(1,1,1) \leq P_{1}^{d}$, then $y_{23}=0$ is a necessary condition for the existence of a profitable deviation for 1 . But this cannot occur in a NE since 3 plays $x_{32}=1$, given $x_{23}^{*}=1$. Therefore, if $P_{1}^{t}(1,1,1) \leq P_{1}^{d}$, there is no NE of $G_{1,3}^{b}\left(\mathbf{x}_{2}^{*}\right)$ which Pareto dominates $\left(\mathbf{x}_{1}^{*}, \mathbf{x}_{3}^{*}\right)$.

- In $G_{2,3}^{b}(1,0)$, we always have $y_{13}=0$. Therefore, $y_{12}=0$ needs to hold for 3 to be active. In the case of $P_{2}^{t}(0,0,1)>P_{2}^{d}$, then $\mathbf{x}_{2}^{\prime}=\mathbf{x}_{3}^{\prime}=(0,1)$ a NE in $G_{2,3}^{b}(1,0)$ which both 2 and 3 prefer to $\left(\mathbf{x}_{2}^{*}, \mathbf{x}_{3}^{*}\right)$. Indeed, given $\mathbf{x}_{3}^{\prime}, 2$ can earn $P_{2}^{t}(0,0,0)$, or 
$P_{2}^{t}(0,0,1)$, or $P_{2}^{d}$, and $P_{2}^{t}(0,0,1)>P_{2}^{d}$ implies that $\mathbf{x}_{2}^{\prime}$ is a best reply to $\mathbf{x}_{3}^{\prime}$. The $\mathrm{NE}\left(\mathbf{x}_{2}^{\prime}, \mathbf{x}_{3}^{\prime}\right)$ Pareto dominates $\left(\mathbf{x}_{2}^{*}, \mathbf{x}_{3}^{*}\right)$, as the profits of 2 and 3 are $P_{2}^{t}(0,0,1)>P_{2}^{d}$ and $P_{3}^{t}(0,0,1)>0$ respectively; as a consequence, $\mathbf{x}^{*}$ is not a CPNE. If instead $P_{2}^{t}(0,0,1) \leq P_{2}^{d}$, then there is no NE in $G_{2,3}^{b}(1,0)$ in which 2 earns more than $P_{2}^{d}$.

Therefore, a CPNE such that 3 is non-active exists if and only if $P_{1}^{d} \geq P_{1}^{t}(1,1,1)$ and $P_{2}^{d} \geq P_{2}^{t}(0,0,1)$. For instance, $\mathbf{x}_{1}^{*}=(1,0), \mathbf{x}_{2}^{*}=(1,1), \mathbf{x}_{3}^{*}=(0,0)$ is a CPNE.

Step 2 A CPNE such that 3 is active exists if and only if (34) is violated. Precisely, the unique CPNE is $\mathbf{x}_{1}=\mathbf{x}_{2}=(0,1), \mathbf{x}_{3}=(1,1)$ if $P_{1}^{d}>P_{1}^{t}(1,1,1)$ and $P_{2}^{t}(0,0,1)>P_{2}^{d}$; the unique CPNE is $\mathbf{x}_{1}=\mathbf{x}_{2}=\mathbf{x}_{3}=(1,1)$ if $P_{1}^{t}(1,1,1)>P_{1}^{d}$.

Proof. If $\mathbf{x}^{*}$ is a CPNE such that 3 is active, then $y^{*}=(0,1,1)$ or $y^{*}=(1,1,1)$.

Consider first the case of $y^{*}=(0,1,1)$.

- About $G_{1,3}^{b}\left(\mathbf{x}_{2}^{*}\right)$ and $G_{2,3}^{b}\left(\mathbf{x}_{1}^{*}\right)$, see footnote 48

- About $G_{1,2}^{b}(1,1)$, it is useful to see the normal form of this game:

\begin{tabular}{|c|c|c|c|c|}
\hline $\mathbf{x}_{1} \backslash \mathbf{x}_{2}$ & $\mathbf{x}_{2}=(1,0)$ & $\mathbf{x}_{2}=(0,1)$ & $\mathbf{x}_{2}=(1,1)$ & $\mathbf{x}_{2}=(0,0)$ \\
\hline$(1,0)$ & $P_{1}^{d}, P_{2}^{d}$ & $P_{1}^{t}(0,0,1), P_{2}^{t}(0,0,1)$ & $P_{1}^{d}, P_{2}^{d}$ & $P_{1}^{t}(0,0,0), P_{2}^{t}(0,0,0)$ \\
\hline$(0,1)$ & $P_{1}^{t}(0,1,0), P_{2}^{t}(0,1,0)$ & $P_{1}^{t}(0,1,1), P_{2}^{t}(0,1,1)$ & $P_{1}^{t}(0,1,1), P_{2}^{t}(0,1,1)$ & $P_{1}^{t}(0,1,0), P_{2}^{t}(0,1,0)$ \\
\hline$(1,1)$ & $P_{1}^{t}(1,1,0), P_{2}^{t}(1,1,0)$ & $P_{1}^{t}(0,1,1), P_{2}^{t}(0,1,1)$ & $P_{1}^{t}(1,1,1), P_{2}^{t}(1,1,1)$ & $P_{1}^{t}(0,1,0), P_{2}^{t}(0,1,0)$ \\
\hline$(0,0)$ & $P_{1}^{t}(0,0,0), P_{2}^{t}(0,0,0)$ & $P_{1}^{t}(0,0,1), P_{2}^{t}(0,0,1)$ & $P_{1}^{t}(0,0,1), P_{2}^{t}(0,0,1)$ & $P_{1}^{t}(0,0,0), P_{2}^{t}(0,0,0)$ \\
\hline
\end{tabular}

There are two kinds of joint deviations in $G_{1,2}^{b}(1,1)$ depending on whether or not a deviation induces exclusion of 3 . First, a joint deviation that excludes 3 yields 1 and 2 more than $P_{1}^{t}(0,1,1)$ and $P_{2}^{t}(0,1,1)$ if and only if $P_{1}^{d}>P_{1}^{t}(0,1,1)$. But since $P_{1}^{t}(1,1,0)>P_{1}^{d}, \mathbf{x}_{1}=\mathbf{x}_{2}=(1,0)$ is not a NE, and exclusion of 3 may occur only with $\mathbf{x}_{1}^{\prime}=(1,0), \mathbf{x}_{2}^{\prime}=(1,1)$, which is a NE if and only if $P_{1}^{d} \geq P_{1}^{t}(1,1,1)$ and $P_{2}^{t}(0,0,1) \leq P_{2}^{d}$. Under these inequalities, the profits of 1 and 2 are higher than $P_{1}^{t}(0,1,1)$ and $P_{2}^{t}(0,1,1)$, respectively, and $\mathbf{x}^{*}$ is not a CPNE. Consider now a joint deviation that does not exclude 3 . Then, $x_{13}=x_{23}=1$ must hold in any NE of $G_{1,2}^{b}(1,1)$ and therefore it must be the case that $\mathbf{x}_{1}^{\prime}=\mathbf{x}_{2}^{\prime}=(1,1)$. Although it Pareto dominates $\left(\mathbf{x}_{1}^{*}, \mathbf{x}_{2}^{*}\right),\left(\mathbf{x}_{1}^{\prime}, \mathbf{x}_{2}^{\prime}\right)$ is a NE if and only if $P_{1}^{t}(1,1,1) \geq P_{1}^{d}$. Summarizing, $\mathbf{x}^{*}$ is a CPNE if and only if $P_{1}^{d}>P_{1}^{t}(1,1,1)$ and $P_{2}^{t}(0,0,1)>P_{2}^{d}$. In order to see that $\mathbf{x}^{*}$ is the unique CPNE in this case, notice that (i) we have proved that $y^{*}=(0,1,1)$, thus it is necessary that $\mathbf{x}_{3}=(1,1)$ and $x_{13}=x_{23}=1$; (ii) if $x_{12}=1$, then 2 has an incentive to play $\mathbf{x}_{2}=(1,1)$, since $P_{2}^{t}(1,1,1)>P_{2}^{t}(0,1,1)$; (iii) if $x_{21}=1$, then 1 has an incentive to play $\mathbf{x}_{1}=(1,0)$, which implies the exclusion of 3 . 
Now consider the case of $y=(1,1,1)$, which requires $\mathbf{x}_{1}^{*}=\mathbf{x}_{2}^{*}=\mathbf{x}_{3}^{*}=(1,1)$. From the normal form of $G_{1,2}^{b}(1,1)$, we see that $\mathbf{x}_{1}^{*}$ is a best reply given $\left(\mathbf{x}_{2}^{*}, \mathbf{x}_{3}^{*}\right)$ if and only if $P_{1}^{t}(1,1,1) \geq P_{1}^{d}$.

- In $G_{1,2}^{b}(1,1)$, there is no NE in which 3 is inactive, as neither $\mathbf{x}_{1}=\mathbf{x}_{2}=(1,0)$ nor $\mathbf{x}_{1}=(1,0), \mathbf{x}_{2}=(1,1)$ is a NE. Therefore $x_{13}=x_{23}=1$, and in no NE the profits of 1 and 2 are higher than $P_{1}^{t}(1,1,1)$.

- In $G_{1,3}^{b}(1,1)$, given that publisher 3 is active, a necessary condition for 1 to earn more than $P_{1}^{t}(1,1,1)$ is that $y_{23}=0$. But this cannot occur since in any NE 3 plays $x_{32}=1$, as $x_{23}^{*}=1$.

- In $G_{2,3}^{b}(1,1)$, given that publisher 3 is active, a necessary condition for 2 to earn more than $P_{2}^{t}(1,1,1)$ is that $y_{13}=0$. But this cannot occur since in any NE 3 plays $x_{31}=1$, as $x_{13}^{*}=1$.

Therefore, $\mathbf{x}_{1}^{*}=\mathbf{x}_{2}^{*}=\mathbf{x}_{3}^{*}=(1,1)$ is a CPNE if and only if $P_{1}^{t}(1,1,1) \geq P_{1}^{d}$.

The proof for the case of $M^{t}(1,0,1)<M \leq M^{t}(1,0,0)$

Step 1 Publisher 3 is active in any CPNE

Proof. Suppose that $\mathbf{x}^{*}$ is such that 3 is inactive; then $\mathbf{x}_{1}^{*}=\mathbf{x}_{2}^{*}=(1,0)$, otherwise $M>M^{t}(y)$ holds if 3 plays $\mathbf{x}_{3}=(1,1)$. However, $\mathbf{x}_{1}^{\prime}=(1,1), \mathbf{x}_{3}^{\prime}=(1,1)$ is a $\mathrm{NE}$ in $G_{1,3}^{b}(1,0)$ which both 1 and 3 prefer to $\left(\mathbf{x}_{1}^{*}, \mathbf{x}_{3}^{*}\right)$. About $\mathbf{x}_{1}^{\prime}$, notice that given $\mathbf{x}_{3}^{\prime}, 1$ can earn $P_{1}^{t}(0,0,0)$, or $P_{1}^{t}(0,1,0)$, or $P_{1}^{d}$, or $P_{1}^{t}(1,1,0)$; since $P_{1}^{t}(1,1,0)>P_{1}^{d}$, we find that $\mathbf{x}_{1}^{\prime}$ is 1 's (unique) best reply to $\mathbf{x}_{3}^{\prime}$. Thus $\left(\mathbf{x}_{1}^{\prime}, \mathbf{x}_{3}^{\prime}\right)$ is a NE of $G_{1,3}^{b}(1,0)$ which Pareto dominates $\left(\mathbf{x}_{1}^{*}, \mathbf{x}_{3}^{*}\right)$ as the profits of 1 and 3 are $P_{1}^{t}(1,1,0)>P_{1}^{d}$ and $P_{3}^{t}(1,1,0)>0$ respectively. As a consequence, $\mathrm{x}^{*}$ is not a CPNE.

Step 2 The unique CPNE is $\mathbf{x}_{1}=\mathbf{x}_{2}=\mathbf{x}_{3}=(1,1)$

Proof. By step 1, publisher 3 is active in any CPNE $\mathbf{x}^{*}$ and thus $y^{*}=(0,1,1)$ or $y^{*}=(1,1,1)$. Furthermore, $y_{12}^{*}=1$ because $\mathbf{x}_{1}=\mathbf{x}_{2}=(1,1)$ is a NE in $G_{1,2}^{b}(1,1)$ which Pareto dominates the NE $\mathbf{x}_{1}=\mathbf{x}_{2}=(0,1)$ as can be seen from the following normal form of the game $G_{1,2}^{b}(1,1)$ :

\begin{tabular}{c|c|c|c|c|}
$\mathbf{x}_{1} \backslash \mathbf{x}_{2}$ & $\mathbf{x}_{2}=(1,0)$ & $\mathbf{x}_{2}=(0,1)$ & $\mathbf{x}_{2}=(1,1)$ & $\mathbf{x}_{2}=(0,0)$ \\
\cline { 2 - 5 }$(1,0)$ & $P_{1}^{d}, P_{2}^{d}$ & $P_{1}^{t}(0,0,1), P_{2}^{t}(0,0,1)$ & $P_{1}^{t}(1,0,1), P_{2}^{t}(1,0,1)$ & $P_{1}^{t}(0,0,0), P_{2}^{t}(0,0,0)$ \\
\cline { 2 - 5 }$(0,1)$ & $P_{1}^{t}(0,1,0), P_{2}^{t}(0,1,0)$ & $P_{1}^{t}(0,1,1), P_{2}^{t}(0,1,1)$ & $P_{1}^{t}(0,1,1), P_{2}^{t}(0,1,1)$ & $P_{1}^{t}(0,1,0), P_{2}^{t}(0,1,0)$ \\
\cline { 2 - 5 }$(1,1)$ & $P_{1}^{t}(1,1,0), P_{2}^{t}(1,1,0)$ & $P_{1}^{t}(0,1,1), P_{2}^{t}(0,1,1)$ & $P_{1}^{t}(1,1,1), P_{2}^{t}(1,1,1)$ & $P_{1}^{t}(0,1,0), P_{2}^{t}(0,1,0)$ \\
\cline { 2 - 5 }$(0,0)$ & $P_{1}^{t}(0,0,0), P_{2}^{t}(0,0,0)$ & $P_{1}^{t}(0,0,1), P_{2}^{t}(0,0,1)$ & $P_{1}^{t}(0,0,1), P_{2}^{t}(0,0,1)$ & $P_{1}^{t}(0,0,0), P_{2}^{t}(0,0,0)$ \\
\cline { 2 - 5 } & & &
\end{tabular}

Therefore, $\mathbf{x}_{1}^{*}=\mathbf{x}_{2}^{*}=\mathbf{x}_{3}^{*}=(1,1)$ is the unique candidate for CPNE, and it is simple to see that it is a NE. In order to see that $\mathbf{x}^{*}$ is a CPNE we examine $G_{1,2}(1,1), G_{1,3}(1,1)$, $G_{2,3}(1,1)$. 
- In $G_{1,2}(1,1), 1$ and 2 can exclude 3 only by playing $\mathbf{x}_{1}=\mathbf{x}_{2}=(1,0)$, but this is not a NE of $G_{1,2}^{b}(1,1)$ since $\mathbf{x}_{1}=(1,1)$ is 1 's unique best reply to $\mathbf{x}_{2}=(1,0)$. If instead 1 and 2 do not play $\mathbf{x}_{1}=\mathbf{x}_{2}=(1,0), 3$ is active and in order for the profit of 1 to be higher than $P_{1}^{t}(1,1,1), y_{23}=0$ is necessary. But this cannot occur since 2 plays $x_{23}=1$ in any NE, as $x_{32}^{*}=1$.

- In $G_{1,3}(1,1), 3$ is certainly active given that $\mathbf{x}_{2}^{*}=(1,1)$, and in order for his profit to be higher than $P_{3}^{t}(1,1,1), y_{12}=0$ is necessary . But this cannot occur since 1 plays $x_{12}=1$ in any NE, as $x_{21}^{*}=1$.

- In $G_{2,3}(1,1), 3$ is certainly active given that $\mathbf{x}_{1}^{*}=(1,1)$, and in order for his profit to be higher than $P_{3}^{t}(1,1,1) y_{12}=0$. is necessary. But this cannot occur since 2 plays $x_{21}=1$ in any NE, as $x_{12}^{*}=1$.

\subsection{Proof of Proposition 4}

Proof of (i). We start by showing that $\left(X_{1}^{*}, X_{2}^{*}, X_{3}^{*}\right)$ is a NE of $G^{h}$. First notice that publisher $i$ 's choice of $x_{i}$ has no effect since $x_{j}^{*}=x_{k}^{*}=0$. Second, publisher $i$ cannot increase his profit by playing $\left(x_{i j}, x_{i k}\right) \neq \mathbf{x}_{i}^{*}$ since $\mathbf{x}^{*}$ is a NE of $G^{b}$.

In order to show that $X^{*}$ is a CPNE of $G^{h}$, consider $G_{i, j}^{h}\left(X_{k}^{*}\right)$ and notice that since $\mathbf{x}^{*}$ is a CPNE in $G^{b}$, there exists no NE $\left(X_{i}, X_{j}\right)$ of $G_{i, j}^{h}\left(X_{k}^{*}\right)$ which is Pareto improving for both $i$ and $j$ if $x_{i}=x_{j}=0$. Furthermore, since $x_{k}^{*}=0$, the only effect of $x_{i}$ and $x_{j}$ can be that of inducing $y_{i j}=1$ (when $x_{i}=x_{j}=1$ ), but $i$ and $j$ can obtain the same effect also by playing $x_{i}=x_{j}=0$ and $\mathbf{x}_{i}$ and $\mathbf{x}_{j}$ such that $x_{i j}=x_{j i}=1$.

Proof of (ii). This proof is split in five steps

Step 1 Suppose that $M$ is between $M^{t}(1,1,1)$ and $M^{t}(1,1,0)$ and $P_{1}^{t}(1,1,1)>P_{1}^{d}$. Then $\hat{X}=\left(\hat{X}_{1}, \hat{X}_{2}, \hat{X}_{3}\right)$ is a CPNE of $G^{h}$, even when no full interconnection CPNE exists in $G^{b}$ Proof. We first show that $\hat{X}$ is a NE of $G^{h}$. Since $\mathbf{x}_{j}=\mathbf{x}_{k}=(0,0)$, publisher $i$ 's choice of $\mathbf{x}_{i}=\left(x_{i j}, x_{i k}\right)$ has no effect; since $\left(x_{1}, x_{2}, x_{3}\right)=(1,1,1)$ is the NE of $G^{m}$, publisher $i$ cannot increase his profit by playing $x_{i}=0$ instead of $x_{i}=1$.

Now we show that $\hat{X}$ is a CPNE of $G^{h}$. Regarding $G_{1,2}^{h}\left(\hat{X}_{3}\right), 1$ and 2 may have the possibility to exclude 3 but then the profit of 1 decreases since $P_{1}^{t}(1,1,1)>P_{1}^{d}$. If instead 3 is not excluded, then $y_{23}=0$ is necessary for 1 to earn more than $P_{1}^{t}(1,1,1)$, but $y_{23}=1$ must hold in any NE of $G_{1,2}^{h}\left(\hat{X}_{3}\right)$ : given that 3 is active and $\hat{x}_{3}=1$, it is profitable for 2 to play $x_{2}=1$ to interconnect with 3 . Regarding $G_{1,3}^{h}\left(\hat{X}_{2}\right)$, in order for 3 to increase his profit it is necessary that he is active, and in this case 1 may increase his profit only if $y_{23}=0$; but $y_{23}=1$ in any NE given that it is profitable for 3 to play $x_{3}=1$ and to interconnect with 2 . Regarding $G_{2,3}^{h}\left(\hat{X}_{1}\right), y_{13}=0$ is necessary for 2 to increase his profit 
but this cannot occur in a NE, given that it is profitable for 3 to play $x_{3}=1$ and to interconnect with $1 .{ }^{50}$

In steps 2-4 below, we show that for $M>M^{t}(1,1,1)$, if $P_{1}^{d}>P_{1}^{t}(1,1,1)$ then the unique CPNE outcome of $G^{h}$ is equal to the CPNE outcome of $G^{b}$.

Step 2 There exists a CPNE in $G^{h}$ such that $y=(0,1,1)$ if and only if it exists in $G^{b}$ Proof. We start by proving that there is no CPNE such that $y=(0,1,1)$ and $x_{3}=1$. Indeed, if $x_{3}=1$ then (independently of $\left.x_{31}, x_{32}\right)$ a NE of $G_{1,2}^{h}\left(X_{3}\right)$ is $X_{1}=X_{2}=(1,0,0)$, and 1 and 2 earn $P_{1}^{t}(1,1,1), P_{2}^{t}(1,1,1)$ which are larger than $P_{1}^{t}(0,1,1), P_{2}^{t}(0,1,1)$. In order to see that $X_{1}=X_{2}=(1,0,0)$ is a NE of $G_{1,2}^{h}\left(X_{3}\right)$, notice that $x_{3}=1$ and $X_{2}=(1,0,0)$ implies $y_{23}=1$ and 1 is unable to exclude 3 ; thus interconnecting with 2 and 3 is the best option for 1 . The same argument applies to 2 and therefore if $y=(0,1,1)$ in a CPNE of $G^{h}$, then $x_{3}=0$ and $\mathbf{x}_{3}=(1,1)$, which in turn implies $x_{13}=x_{23}=1$. In order to verify whether there exists a CPNE of this sort, it is enough to consider only $G_{1,2}^{h}\left(X_{3}\right)$; and here $x_{1}, x_{2}$ have the same effect as $x_{12}, x_{21}$ (that is, we can think without loss of generality that $\left.x_{1}=x_{2}=0\right)$. Hence, the same result as in $G^{b}$ is obtained.

\section{Step 3 There exists a CPNE in $G^{h}$ which makes 3 inactive if and only if it} exists in $G^{b}$ Proof. Consider first the case of $M^{t}(1,1,1)<M \leq M^{t}(1,1,0)$. We prove that there exists a CPNE in $G^{h}$ in which 3 is inactive if and only if $P_{1}^{d}>P_{1}^{t}(0,1,0)$. In the case of $P_{1}^{d}>P_{1}^{t}(0,1,0)$, the existence of such a CPNE is delivered by Propositions 3(iiia) and 4(i). If instead $P_{1}^{t}(0,1,0)>P_{1}^{d}$, we show that there is no CPNE in which 3 is excluded. We distinguish three cases, depending on the values of $x_{2}, x_{23}$.

If $X_{2}$ is such that $x_{2}=1$ (independently of $x_{23}$ ) then $X_{1}=X_{3}=(1,0,0)$ is a NE of $G_{1,3}^{h}\left(X_{2}\right)$ and both 1 and 3 improve their profits as $P_{1}^{t}(1,1,1)>P_{1}^{t}(0,1,0)>P_{1}^{d}$.

If $x_{2}=0, x_{23}=1$, then exclusion of 3 requires $x_{12}=x_{21}=1$ but in $G_{1,3}^{h}\left(X_{2}\right), X_{1}=$ $(0,1,1), X_{3}=(0,1,1)$ is a NE and both 1 and 3 improve their profits as $P_{1}^{t}(1,1,1)>P_{1}^{d}$. If $x_{2}=x_{23}=0$, then $X_{1}=(0,0,1), X_{3}=(0,1,0)$ is a NE in $G_{1,3}^{h}\left(X_{2}\right)$ and both 1 and 3 improve their profits as $P_{1}^{t}(0,1,0)>P_{1}^{d}$.

Now consider the case of $M^{t}(1,1,0)<M<M^{t}(1,0,1)$. We prove that there exists a CPNE of $G^{h}$ in which 3 is inactive if and only if $P_{1}^{d}>P_{1}^{t}(1,1,1)$, as in $G^{b}$. If $P_{1}^{d}>$ $P_{1}^{t}(1,1,1)$, then existence follows from Propositions 3 (iva) and 4(i). If instead $P_{1}^{t}(1,1,1)>$ $P_{1}^{d}$, we show that there exists no CPNE in which 3 is excluded; notice that for 3 to be inactive it is necessary that $y_{12}=1$ and $y_{13}=0$, which implies $x_{12}=x_{21}=1, x_{1}=0$, $x_{13}=0$.

\footnotetext{
${ }^{50}$ This proof does not require that the inequality $M \leq M(1,1,0)$ is satisfied, but if $M>M(1,1,0)$ then we know from Propositions 3(ivb) and 4(i) that a full interconnection CPNE exists in $G^{h}$ if $P_{1}^{t}(1,1,1)>$ $P_{1}^{d}$.
} 
If $x_{2}=1$ (independently of $x_{23}$ ), a NE in $G_{1,3}^{h}\left(X_{2}\right)$ is $X_{1}=X_{3}=(1,0,0)$ and both 1 and 3 improve their profits since $P_{1}^{t}(1,1,1)>P_{1}^{d}$.

If $x_{2}=x_{23}=0$, then $X_{1}=(0,1,1), X_{3}=(0,1,0)$ is a NE of $G_{1,3}^{h}\left(X_{2}\right)$ and both 1 and 3 improve their profits as $P_{1}^{t}(1,1,0)>P_{1}^{t}(1,1,1)>P_{1}^{d}$.

If $x_{2}=0, x_{23}=1$, then $X_{1}=(0,1,1), X_{3}=(0,1,1)$ is a NE in $G_{1,3}^{h}\left(X_{2}\right)$ and both 1 and 3 improve their profits.

Finally, the case of $M^{t}(1,0,1)<M \leq M^{t}(1,0,0)$ is straightforward, as the exclusion of 3 requires $X_{1}=X_{2}=(0,1,0)$, but $X_{1}=(0,1,1), X_{3}=(0,1,0)$ is a NE of $G_{1,3}^{h}\left(X_{2}\right)$ and both 1 and 3 improve their profits. Thus, there exists no CPNE in which 3 is inactive.

Step 4 For $M$ between $M^{t}(1,1,1)$ and $M^{t}(1,0,1)$ there exists no CPNE with full interconnection in $G^{h}$ if $P_{1}^{d}>P_{1}^{t}(1,1,1) \quad$ Proof. Consider first the case of $M$ between $M^{t}(1,1,1)$ and $M^{t}(1,1,0)$. No full interconnection exists since $X_{1}=X_{2}=(0,1,0)$ a $\mathrm{NE}$ in $G_{1,2}^{h}\left(X_{3}\right)$ and yields $P_{1}^{d}, P_{2}^{d}$, so that both 1 and 2 improve. Precisely, $X_{1}=(0,1,0)$ is a best reply for 1 because there is no $y$ which makes 3 active and yields 1 more than $P_{1}^{d}$, given that $P_{1}^{d}>P_{1}^{t}(1,1,1)>P_{1}^{t}(0,1,0) ; X_{2}$ is a best reply for 2 since $P_{2}^{d}>P_{2}^{t}(0,0,1)$.

In the case of $M$ between $M^{t}(1,1,0)$ and $M^{t}(1,0,1)$, full interconnection requires either $x_{3}=1$, or $\left(x_{3}=0, x_{31}=x_{32}=1\right)$. In the first case, in $G_{1,2}^{h}\left(X_{3}\right), X_{1}=(0,1,0)$, $X_{2}=(1,1,0)$ leads to $y=(1,0,1)$ and exclusion of 3 . It is a NE since (i) for $1, y_{23}=1$ and $P_{1}^{d}>P_{1}^{t}(1,1,1) \geq P_{1}^{t}\left(y_{12}, y_{13}, 1\right)$; (ii) for $2, y_{13}=0$ and $P_{2}^{d}>P_{2}^{t}(0,0,1)$. In the second case, in $G_{1,2}^{h}\left(X_{3}\right), X_{1}=(0,1,0), X_{2}=(0,1,1)$ leads to $y=(1,0,1)$ and exclusion of 3 ; it is a NE by the same arguments described above.

Therefore, for $M$ between $M^{t}(1,1,1)$ and $M^{t}(1,0,1)$, there is no full interconnection CPNE in $G^{h}$ if $P_{1}^{d}>P_{1}^{t}(1,1,1)$. In the case of $M$ between $M^{t}(1,0,1)$ and $U-I_{12}$ we already have full interconnection in $G^{b}$ (and hence in $G^{h}$ ) independently of whether $P_{1}^{d}>P_{1}^{t}(1,1,1)$ holds or not.

Step 5 For $M$ smaller than $M^{t}(1,1,1), G^{h}$ has the same CPNE outcome as $G^{b}$ Proof. If $M^{t}(0,1,0)<M \leq M^{t}(1,1,1)$ and $P_{1}^{t}(0,1,1)>P_{1}^{d}$, then we can argue as in the proof of Proposition 3 to show that 3 is active in any CPNE. Indeed, if $\left(X_{1}^{*}, X_{2}^{*}, X_{3}^{*}\right)$ is a strategy profile such that 3 is inactive, then $X_{1}^{\prime}=(0,0,1), X_{3}^{\prime}=(1,1,1)$ is a NE in $G_{1,3}^{h}\left(X_{2}^{*}\right)$ which increases the payoff of both 1 and 3 . Precisely, $X_{3}^{\prime}=(1,1,1)$ is a weakly dominant strategy for 3 . If 1 plays $x_{1}=1$, then either 3 is excluded (if $x_{2}^{*}=1$ ) or $x_{1}=1$ is equivalent to $x_{13}=1$; since $P_{1}^{t}(0,1,1)>P_{1}^{d}, 1$ prefers to play $X_{1}^{\prime}=(0,0,1)$ rather than some $X_{1}$ such that $x_{1}=1$ (in the same way as we prove that 1 prefers to play $x_{12}=0$ rather than $\left.x_{12}=1\right)$.

If $M^{t}(0,1,0)<M \leq M^{t}(1,1,1)$ and $P_{1}^{t}(0,1,1)<P_{1}^{d}$, then 3 is inactive in any CPNE. If 3 is active, then it is necessary that $x_{1}=0$; otherwise, publisher 2 would play $x_{2}=1$ in 
order to exclude 3. Likewise, it is necessary that $x_{2}=0$; otherwise, 3 would play $x_{3}=1$ in order to interconnect with 2 , and then 1 would also play $x_{1}=1$ in order to exclude 3 given that $P_{1}^{t}(0,1,1)<P_{1}^{d}$. It is also necessary that $y_{12}=0$, and by arguing as in Lemma 5 we can prove that $y=(0,1,1)$ in any CPNE in which 3 is active. However, in $G_{1,2}^{h}\left(X_{3}\right)$ there exists a $\mathrm{NE} X_{1}=X_{2}=(0,1,1)$ (we can argue like in the proof of Proposition 3 ) which excludes 3 and makes better off both 1 and 2 .

\subsection{Proof of Proposition 5}

In the proof, we consider five different cases depending on the level of $M$.

\subsubsection{When $M^{t}(0,1,0)<M \leq M^{t}(0,0,1)$}

Proof. For these values of $M$, the profiles $x=(1,1,1),{ }^{51} x=(1,1,0),{ }^{52} x=(0,1,1)$ are not NE. Hence, the platform can make money only by inducing the publishers to play $x=$ $(1,0,1)$. The publishers' profits with $x=(1,0,1)$ are $P_{1}^{t}(0,1,0)-f_{1}, P_{2}^{t}(0,1,0), P_{3}^{t}(0,1,0)-$ $f_{3}$, and we prove that $x=(1,0,1)$ is the unique CPNE if

$$
f_{1}<P_{1}^{t}(0,1,0)-P_{1}^{d},{ }^{53} \quad f_{2} \text { large, } \quad f_{3}<P_{3}^{t}(0,1,0)
$$

Indeed, it is readily seen that $x=(1,0,1)$ is NE when (35) is satisfied: (i) by playing $x_{1}=0,1$ could exclude 3 and obtain $P_{1}^{d}$; (ii) 2 is not going to interconnect since $f_{2}$ is large; (iii) 3 is not active and obtains 0 if he does not interconnect. In order to verify that $x=(1,0,1)$ is also a CPNE, we need to examine $G_{1,2}^{m}(1), G_{1,3}^{m}(0), G_{2,3}^{m}(1)$, but notice that the analysis is simple for $G_{1,2}^{m}(1), G_{2,3}^{m}(1)$, as $f_{2}$ large implies that 2 plays $x_{2}=0$; thus the unique NE of $G_{1,2}^{m}(1)$ is $\left(x_{1}, x_{2}\right)=(1,0)$ and the unique NE of $G_{2,3}^{m}(1)$ is $\left(x_{2}, x_{3}\right)=(0,1)$ :

$$
\begin{aligned}
& \begin{array}{cccc} 
& \multicolumn{1}{c}{1 \backslash 2} & x_{2}=0 & x_{2}=1 \\
\cline { 3 - 4 } G_{1,2}^{m}(1): & x_{1}=0 & P_{1}^{d}, P_{2}^{d} & P_{1}^{d}, P_{2}^{d}-f_{2} \\
\cline { 3 - 4 } & x_{1}=1 & P_{1}^{t}(0,1,0)-f_{1}, P_{2}^{t}(0,1,0) & P_{1}^{d}-f_{1}, P_{2}^{d}-f_{2} \\
\cline { 3 - 4 } & &
\end{array} \\
& 2 \backslash 3 \quad x_{3}=0 \quad x_{3}=1 \\
& \begin{array}{cc|c|c|}
G_{2,3}^{m}(1): & x_{2}=0 & P_{2}^{d}, 0 & P_{2}^{t}(0,1,0), P_{3}^{t}(0,1,0)-f_{3} \\
\cline { 3 - 4 } & x_{2}=1 & P_{2}^{d}-f_{2}, 0 & P_{2}^{d}-f_{2},-f_{3} \\
\cline { 3 - 4 }
\end{array}
\end{aligned}
$$

Things are less obvious in $G_{1,3}^{m}(0)$ :

$$
\begin{array}{ccccc}
\multicolumn{1}{c}{1 \backslash 3} & x_{3}=0 & x_{3}=1 \\
\cline { 3 - 4 } G_{1,3}^{m}(0): & x_{1}=0 & P_{1}^{d}, 0 & P_{1}^{d},-f_{3} \\
\cline { 3 - 4 } & x_{1}=1 & P_{1}^{d}-f_{1}, 0 & P_{1}^{t}(0,1,0)-f_{1}, P_{3}^{t}(0,1,0)-f_{3} \\
\cline { 3 - 4 } & & &
\end{array}
$$

\footnotetext{
${ }^{51}$ Publisher 3 will not pay for interconnection, given that later on he will be inactive.

${ }^{52}$ There is no point in interconnection for publisher 2 , given that 3 will be inactive anyway.
} 
Here both $\left(x_{1}, x_{3}\right)=(1,1)$ and $\left(x_{1}, x_{3}\right)=(0,0)$ are NE, but the former strictly Pareto dominates the latter since $f_{1}<P(0,1,0)-P_{1}^{d}$ and $f_{3}<P_{3}^{t}(0,1,0)$. Hence $x=(1,0,1)$ is a CPNE, and is actually the unique CPNE given (35). Indeed, the only possible alternative is $x=(0,0,0)$ [we mentioned above that $x=(1,1,1), x=(1,1,0), x=(0,1,1)$ are not even NE], but $G_{1,3}^{m}(0)$ reveals that $x=(0,0,0)$ is not a CPNE because $\left(x_{1}, x_{3}\right)=(1,1)$ is a NE of $G_{1,3}^{m}(0)$ which strictly Pareto dominates $\left(x_{1}, x_{3}\right)=(0,0)$.

Let $R$ denote the revenue of the platform. When $M^{t}(0,1,0)<M \leq M^{t}(0,0,1)$, $R$ can be made arbitrarily close to $P_{1}^{t}(0,1,0)+P_{3}^{t}(0,1,0)-P_{1}^{d}$, and thus for simplicity we consider $R$ equal to $P_{1}^{t}(0,1,0)+P_{3}^{t}(0,1,0)-P_{1}^{d} \cdot{ }^{54}$ The same remark applies in the following of the analysis.

\subsubsection{When $M^{t}(0,0,1)<M \leq M^{t}(0,0,0)$}

Proof. For these values of $M$ the profiles $x=(1,1,1)$ and $x=(1,1,0)$ are not NE, and thus the platform can make money only by inducing the publishers to play $x=(1,0,1)$ or $x=(0,1,1)$.

First we show that if the fees satisfy $(35)$, then $x=(1,0,1)$ is the unique CPNE. If (35) holds, it is still true (as above) that $x=(1,0,1)$ is a CPNE since the only difference in $G_{1,2}^{m}(1), G_{1,3}^{m}(0), G_{2,3}^{m}(1)$ with respect to the above analysis occurs in $G_{1,2}^{m}(1)$, when $x_{1}=0$ and $x_{2}=1$, but $\left(x_{1}, x_{2}\right)=(0,1)$ is not a NE of $G_{1,2}^{m}(1)$ given $f_{2}$ large. Furthermore, $G_{1,3}^{m}(0)$ still reveals that $x=(0,0,0)$ is not a CPNE, and $x=(0,1,1)$ is not a NE given that $f_{2}$ is large. Hence, the revenue the platform can make by inducing $x=(1,0,1)$ is (again) $P_{1}^{t}(0,1,0)+P_{3}^{t}(0,1,0)-P_{1}^{d}$.

If

$$
f_{1} \text { large, } \quad f_{2}<P_{2}^{t}(0,0,1)-P_{2}^{d}, \quad f_{3}<P_{3}^{t}(0,0,1)
$$

holds, then the profile $x=(0,1,1)$ is the unique CPNE. The publishers' profits with $x=(0,1,1)$ are $P_{1}^{t}(0,0,1), P_{2}^{t}(0,0,1)-f_{2}, P_{3}^{t}(0,0,1)-f_{3}$ and we can see that it is a NE if (36) is satisfied: (i) 1 is not going to interconnect since $f_{1}$ is large; (ii) by playing $x_{2}=0,2$ could exclude 3 and obtain $P_{2}^{d}$; (iii) 3 is not active and obtains 0 if he does not interconnect. In order to verify that $x=(0,1,1)$ is also a CPNE, we need to examine $G_{1,2}^{m}(1), G_{1,3}^{m}(1), G_{2,3}^{m}(0)$, but notice that the analysis is simple for $G_{1,2}^{m}(1), G_{1,3}^{m}(1)$, as $f_{1}$ large implies that 1 plays $x_{1}=0$; thus the unique NE of $G_{1,2}^{m}(1)$ is $\left(x_{1}, x_{2}\right)=(0,1)$ and the unique NE of $G_{1,3}^{m}(1)$ is $\left(x_{1}, x_{3}\right)=(0,1)$.

\footnotetext{
${ }^{54}$ Notice that $R$ cannot be larger than $P_{1}(0,1,0)-\hat{P}_{1}+P_{3}(0,1,0)$, because if $f_{1}>P_{1}(0,1,0)-\hat{P}_{1}$ and/or $f_{3}>P_{3}(0,1,0)$ then $x=(1,0,1)$ is not a NE.
} 
Things are less obvious in $G_{2,3}^{m}(0)$ :

$$
\begin{array}{rc|c|c|}
\multicolumn{1}{c}{2 \backslash 3} & x_{3}=0 & x_{3}=1 \\
\cline { 3 - 4 } G_{2,3}^{m}(0): & x_{2}=0 & P_{2}^{d}, 0 & P_{2}^{d},-f_{3} \\
\cline { 3 - 4 } & x_{2}=1 & P_{2}^{d}-f_{2}, 0 & P_{2}^{t}(0,0,1)-f_{2}, P_{3}^{t}(0,0,1)-f_{3} \\
\cline { 3 - 4 } & &
\end{array}
$$

Here both $\left(x_{2}, x_{3}\right)=(1,1)$ and $\left(x_{2}, x_{3}\right)=(0,0)$ are NE, but the former strictly Pareto dominates the latter since $f_{2}<P_{2}^{t}(0,0,1)-P_{2}^{d}$ and $f_{3}<P_{3}^{t}(0,0,1)$. Hence $x=(0,1,1)$ is a CPNE, and is actually the unique CPNE given (36) because the only possible alternatives are $x=(0,0,0)$ and $x=(1,0,1)$, but $G_{2,3}^{m}(0)$ reveals that $x=(0,0,0)$ is not a CPNE, and $x=(1,0,1)$ is not a NE given that $f_{1}$ is large. Therefore, $R$ is (arbitrarily close to) $P_{2}^{t}(0,0,1)+P_{3}^{t}(0,0,1)-P_{2}^{d}$ if the platform induces $x=(0,1,1)$.

What does the platform prefer between $x=(1,0,1)$ and $x=(0,1,1)$ ? We need to compare $P_{1}^{t}(0,1,0)+P_{3}^{t}(0,1,0)-P_{1}^{d}=P_{1}^{t}(0,0,0)+P_{3}^{t}(0,0,0)-P_{1}^{d}+\frac{2}{3} I_{13}$ and $P_{2}^{t}(0,0,1)+$ $P_{3}^{t}(0,0,1)-P_{2}^{d}=P_{2}^{t}(0,0,0)+P_{3}^{t}(0,0,0)-P_{2}^{d}+\frac{2}{3} I_{23}$. Since $P_{1}^{t}(0,0,0)+P_{3}^{t}(0,0,0)-P_{1}^{d}=$ $P_{2}^{t}(0,0,0)+P_{3}^{t}(0,0,0)-P_{2}^{d}$, we conclude that the platform prefers $x=(1,0,1)$ because inducing interconnection of 1 and 3 generates more surplus to 1 and 3 (which can be extracted) than inducing interconnection of 2 and 3 does to 2 and 3.

11.10.3 When $M^{t}(0,0,0)<M<M^{t}(1,1,1)$

Proof. For these values of $M$, the profile $x=(1,1,1)$ is not a NE, but $x=(1,1,0)$, $x=(1,0,1), x=(0,1,1)$ are each a CPNE with a suitable profile of fees.

In particular, the profile $x=(1,0,1)$ is the unique CPNE if

$$
f_{1}<\frac{1}{3} I_{13}, \quad f_{2} \text { large }, \quad f_{3}<\frac{1}{3} I_{13}
$$

This claim is proved by arguing essentially as in the proof of Step 1. We below report only $G_{1,3}^{m}(0)$ because $f_{2}$ large implies that 2 plays $x_{2}=0$, thus $\left(x_{1}, x_{2}\right)=(1,0)$ is the unique NE of $G_{1,2}^{m}(1)$ and $\left(x_{2}, x_{3}\right)=(0,1)$ is the unique NE of $G_{2,3}^{m}(1)$ :

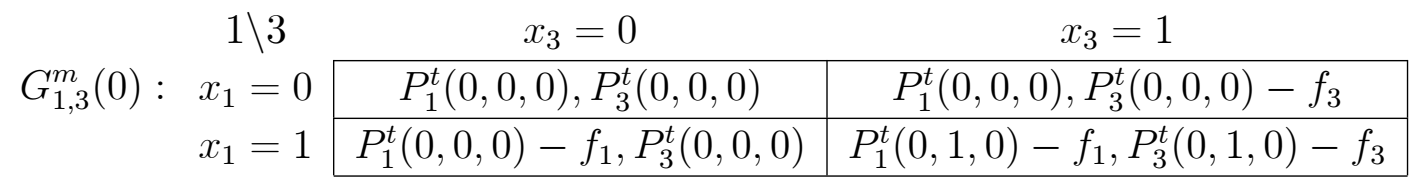

In this case $R=\frac{2}{3} I_{13}$ (actually, arbitrarily close to $\frac{2}{3} I_{13}$ ).

The profile $x=(0,1,1)$ is the unique CPNE if

$$
f_{1} \text { is large, } \quad f_{2}<\frac{1}{3} I_{23}, \quad f_{3}<\frac{1}{3} I_{23}
$$


This claim is proved by arguing essentially as in the proof of Step 2. We below report only $G_{2,3}^{(0)}$ because $f_{1}$ large implies that 1 plays $x_{1}=0$, thus $\left(x_{1}, x_{2}\right)=(0,1)$ is the unique $\mathrm{NE}$ of $G_{1,2}^{m}(1)$ and $\left(x_{1}, x_{3}\right)=(0,1)$ is the unique NE of $G_{1,3}^{m}(1)$ :

$$
\begin{array}{ccccc} 
& \multicolumn{2}{c}{2 \backslash 3} & x_{3}=0 & x_{3}=1 \\
\cline { 3 - 4 } G_{2,3}^{m}(0): & x_{2}=0 & P_{2}^{t}(0,0,0), P_{3}^{t}(0,0,0) & P_{2}^{t}(0,0,0), P_{3}^{t}(0,0,0)-f_{3} \\
\cline { 3 - 4 } & x_{2}=1 & P_{2}^{t}(0,0,0)-f_{2}, P_{3}^{t}(0,0,0) & P_{2}^{t}(0,0,1)-f_{2}, P_{3}^{t}(0,0,1)-f_{3} \\
\cline { 3 - 4 } & &
\end{array}
$$

In this case $R=\frac{2}{3} I_{23}$.

The profile $x=(1,1,0)$ is the unique CPNE if

$$
f_{1}<P_{1}^{d}-P_{1}^{t}(0,0,0), \quad f_{2}<P_{2}^{d}-P_{2}^{t}(0,0,0), \quad f_{3} \text { is large }
$$

In order to prove this claim, we study $G_{1,2}^{m}(0), G_{1,3}^{m}(1), G_{2,3}^{m}(1)$ :

$$
\begin{array}{cc|c|c|}
\multicolumn{2}{c}{1 \backslash 2} & \multicolumn{1}{c}{x_{2}=0} & x_{2}=1 \\
\cline { 3 - 4 } G_{1,2}^{m}(0): & x_{1}=0 & P_{1}^{t}(0,0,0), P_{2}^{t}(0,0,0) & P_{1}^{t}(0,0,0), P_{2}^{t}(0,0,0)-f_{2} \\
\cline { 3 - 4 } & x_{1}=1 & P_{1}^{t}(0,0,0)-f_{1}, P_{2}^{t}(0,0,0) & P_{1}^{d}-f_{1}, P_{2}^{d}-f_{2} \\
\cline { 3 - 4 } & &
\end{array}
$$

Here $\left(x_{1}, x_{2}\right)=(0,0)$ and $\left(x_{1}, x_{2}\right)=(1,1)$ are both NE, but the latter NE strictly Pareto dominates the former [this implies that $x=(0,0,0)$ is not a CPNE].

$$
\begin{array}{rcccc} 
& \multicolumn{1}{c}{1 \backslash 3} & x_{3}=0 & x_{3}=1 \\
\cline { 3 - 4 } G_{1,3}^{m}(1): & x_{1}=0 & P_{1}^{t}(0,0,0), P_{3}^{t}(0,0,0) & P_{1}^{t}(0,0,1), P_{3}^{t}(0,0,1)-f_{3} \\
\cline { 3 - 4 } & x_{1}=1 & P_{1}^{d}-f_{1}, 0 & P_{1}^{d}-f_{1},-f_{3} \\
\cline { 3 - 4 } & & &
\end{array}
$$

Here $x_{1}=1$ is strictly dominant for publisher 1 and $x_{3}=0$ is strictly dominant for publisher 3 ; hence $\left(x_{1}, x_{3}\right)=(1,0)$ is the unique NE.

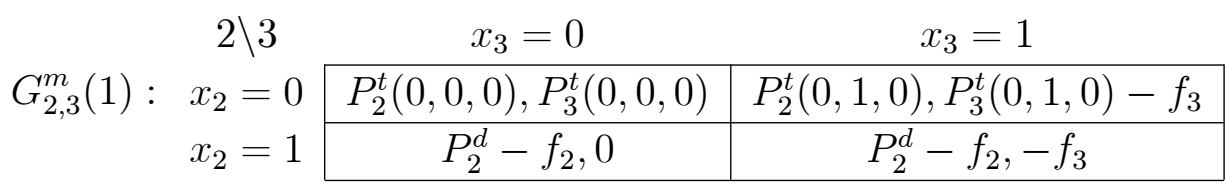

Here $x_{2}=1$ is strictly dominant for firm 2 and $x_{3}=0$ is strictly dominant for firm 3 ; hence $\left(x_{2}, x_{3}\right)=(1,0)$ is the unique NE.

Since $f_{3}$ is large, it is obvious that $x=(0,1,1)$ and $x=(1,0,1)$ are not NE with the fees in (39). Hence $R=M-P_{1}^{t}(0,0,0)-P_{2}^{t}(0,0,0)=P_{3}^{t}(0,0,0)=U_{3}+\frac{1}{3}(M-U)$.

In order to find the optimal fees, the platform needs to compare $P_{3}^{t}(0,0,0)$ with $\frac{2}{3} I_{13}$ (since $\left.\frac{2}{3} I_{13}>\frac{2}{3} I_{23}\right)$. For $M$ close to $M^{t}(0,0,0), P_{3}^{t}(0,0,0)$ is close to 0 ; for $M$ close to $M^{t}(1,1,1)$, instead, $P_{3}^{t}(0,0,0)$ is close to $\frac{2}{3} I_{12}-\frac{1}{3} I_{13}-\frac{1}{3} I_{23}$ which is larger than $\frac{2}{3} I_{13}$ for some parameters. 
11.10.4 When $M^{t}(1,1,1)<M \leq M^{t}(1,0,0)$

For these values of $M$, we can argue like in step 3 to show that the platform

- can earn $\frac{2}{3} I_{13}$ by inducing $x=(1,0,1)$ with the fees in $(37)$;

- can earn $\frac{2}{3} I_{23}$ by inducing $x=(0,1,1)$ with the fees in $(38)$;

- can earn $P_{3}^{t}(0,0,0)$ by inducing $x=(1,1,0)$ with the fees in $(39)$.

Now we study the revenue the platform can make by inducing $x=(1,1,1)$. This profile is a NE if and only if

$$
f_{1} \leq \frac{1}{3}\left(I_{12}+I_{13}\right), \quad f_{2} \leq \frac{1}{3}\left(I_{12}+I_{23}\right), \quad f_{3} \leq P_{3}^{t}(1,1,1)
$$

In order to see whether $x=(1,1,1)$ is a CPNE, we study $G_{1,2}^{m}(1), G_{1,3}^{m}(1), G_{2,3}^{m}(1)$ :

$$
\begin{aligned}
& \begin{array}{rr}
x_{2}=0 & x_{2}=1
\end{array} \\
& \begin{array}{|cc|c|c|}
\cline { 2 - 4 } G_{1,2}^{m}(1): & x_{1}=0 & P_{1}^{t}(0,0,0), P_{2}^{t}(0,0,0) & P_{1}^{t}(0,0,1), P_{2}^{t}(0,0,1)-f_{2} \\
\cline { 3 - 4 } & x_{1}=1 & P_{1}^{t}(0,1,0)-f_{1}, P_{2}^{t}(0,1,0) & P_{1}^{t}(1,1,1)-f_{1}, P_{2}^{t}(1,1,1)-f_{2} \\
\cline { 2 - 4 }
\end{array}
\end{aligned}
$$

here $\left(x_{1}, x_{2}\right)=(1,1)$ is a NE, but for some values of $f_{1}, f_{2}$ consistent with (40) we find that also $\left(x_{1}, x_{2}\right)=(0,0)$ is a NE and strictly Pareto dominates $\left(x_{1}, x_{2}\right)=(1,1)$; in these cases $x=(1,1,1)$ is not a CPNE. Precisely, $x=(1,1,1)$ is not a CPNE if and only if $f_{1} \geq \frac{1}{3} I_{13}, f_{2} \geq \frac{1}{3} I_{23}$ and $f_{1}>\frac{1}{3}\left(I_{12}+I_{13}-2 I_{23}\right), f_{2}>\frac{1}{3}\left(I_{12}+I_{23}-2 I_{13}\right)$.

$$
\begin{array}{cc|c|c|}
\multicolumn{1}{c}{1 \backslash 3} & \multicolumn{2}{c}{x_{3}=0} & x_{3}=1 \\
\cline { 3 - 3 } G_{1,3}^{m}(1): & x_{1}=0 & P_{1}^{t}(0,0,0), P_{3}^{t}(0,0,0) & P_{1}^{t}(0,0,1), P_{3}^{t}(0,0,1)-f_{3} \\
\cline { 3 - 4 } & x_{1}=1 & P_{1}^{d}-f_{1}, 0 & P_{1}^{t}(1,1,1)-f_{1}, P_{3}^{t}(1,1,1)-f_{3} \\
\cline { 3 - 4 } & &
\end{array}
$$

Here $\left(x_{1}, x_{3}\right)=(1,1)$ is a NE, but for some values of $f_{1}, f_{3}$ consistent with (40) we find that also $\left(x_{1}, x_{3}\right)=(0,0)$ is a NE and strictly Pareto dominates $\left(x_{1}, x_{3}\right)=(1,1)$; in these cases $x=(1,1,1)$ is not a CPNE. Precisely, $x=(1,1,1)$ is not a CPNE if and only if $f_{1} \geq P_{1}^{d}-P_{1}^{t}(0,0,0), f_{3} \geq \frac{1}{3} I_{23}$ and $f_{1}>\frac{1}{3}\left(I_{12}+I_{13}-2 I_{23}\right)$.

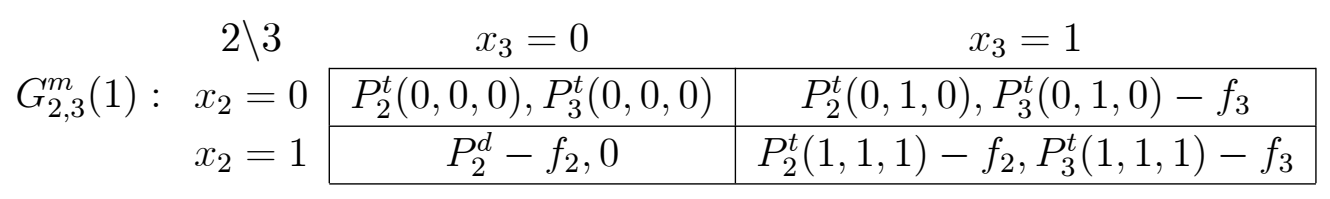

Here $\left(x_{2}, x_{3}\right)=(1,1)$ is a NE, but for some values of $f_{2}, f_{3}$ consistent with (40) we find that also $\left(x_{2}, x_{3}\right)=(0,0)$ is a NE and strictly Pareto dominates $\left(x_{2}, x_{3}\right)=(1,1)$. Hence, $x=(1,1,1)$ is not a CPNE if $f_{2} \geq P_{2}^{d}-P_{2}^{t}(0,0,0), f_{3} \geq \frac{1}{3} I_{13}$ and $f_{2}>\frac{1}{3}\left(I_{12}+I_{23}-2 I_{13}\right)$, 
but actually the first condition implies the third one for any $M$ between $M^{t}(1,1,1)$ and $M^{t}(1,0,0)$.

Therefore, $x=(1,1,1)$ is not a CPNE if at least one of the three sets of conditions (41)-(43) below is satisfied. Equivalently, $x=(1,1,1)$ is a CPNE if and only if the following three sets of conditions are all violated [and (40) is satisfied]

$$
\begin{aligned}
& f_{1} \geq \frac{1}{3} I_{13}, \quad f_{1}>\frac{1}{3}\left(I_{12}+I_{13}-2 I_{23}\right) \quad \text { and } \quad f_{2} \geq \frac{1}{3} I_{23}, \quad f_{2}>\frac{1}{3}\left(I_{12}+I_{23}-2 I_{(3(3) 1)}\right) \\
& f_{1} \geq P_{1}^{d}-P_{1}^{t}(0,0,0), \quad f_{1}>\frac{1}{3}\left(I_{12}+I_{13}-2 I_{23}\right) \quad \text { and } \quad f_{3} \geq \frac{1}{3} I_{23} \\
& f_{2} \geq P_{2}^{d}-P_{2}^{t}(0,0,0) \quad \text { and } \quad f_{3} \geq \frac{1}{3} I_{13}
\end{aligned}
$$

Furthermore, for some fees which violate (41)-(43), we have that $x=(0,0,0)$ is a CPNE. Precisely, we know that $x=(0,0,0)$ is always a NE and we need to study $G_{1,2}^{m}(0), G_{1,3}^{m}(0), G_{2,3}^{m}(0)$ to establish whether it is also a CPNE

$$
\begin{aligned}
& \begin{array}{ccccc|} 
& \multicolumn{2}{c}{1 \backslash 2} & x_{2}=0 & x_{2}=1 \\
\cline { 3 - 4 } G_{1,2}^{m}(0): & x_{1}=0 & P_{1}^{t}(0,0,0), P_{2}^{t}(0,0,0) & P_{1}^{t}(0,0,0), P_{2}^{t}(0,0,0)-f_{2} \\
\cline { 3 - 4 } & x_{1}=1 & P_{1}^{t}(0,0,0)-f_{1}, P_{2}^{t}(0,0,0) & P_{1}^{d}-f_{1}, P_{2}^{d}-f_{2} \\
\cline { 3 - 4 } & &
\end{array} \\
& 1 \backslash 3 \quad x_{3}=0 \quad x_{3}=1 \\
& \begin{array}{|cc|c|c|}
\cline { 3 - 4 } G_{1,3}^{m}(0): & x_{1}=0 & P_{1}^{t}(0,0,0), P_{3}^{t}(0,0,0) & P_{1}^{t}(0,0,0), P_{3}^{t}(0,0,0)-f_{3} \\
\cline { 3 - 4 } & x_{1}=1 & P_{1}^{t}(0,0,0)-f_{1}, P_{3}^{t}(0,0,0) & P_{1}^{t}(0,1,0)-f_{1}, P_{3}^{t}(0,1,0)-f_{3} \\
\cline { 3 - 4 }
\end{array} \\
& \begin{array}{ccc|c|c|} 
& & 2 \backslash 3 & x_{3}=0 & x_{3}=1 \\
\cline { 3 - 4 } G_{2,3}^{m}(0) & : & x_{2}=0 & P_{2}^{t}(0,0,0), P_{3}^{t}(0,0,0) & P_{2}^{t}(0,0,0), P_{3}^{t}(0,0,0)-f_{3} \\
\cline { 3 - 4 } & x_{2}=1 & P_{2}^{t}(0,0,0)-f_{2}, P_{3}^{t}(0,0,0) & P_{2}^{t}(0,0,1)-f_{2}, P_{3}^{t}(0,0,1)-f_{3} \\
\cline { 3 - 4 } & &
\end{array}
\end{aligned}
$$

In $G_{1,2}^{m}(0)$ there exists a NE which strictly Pareto dominates $\left(x_{1}, x_{2}\right)=(0,0)$ if and only if $f_{1}<P_{1}^{d}-P_{1}^{t}(0,0,0)$ and $f_{2}<P_{2}^{d}-P_{2}^{t}(0,0,0)$. Likewise, in $G_{1,3}^{m}(0)$ there exists a NE which strictly Pareto dominates $\left(x_{1}, x_{3}\right)=(0,0)$ if and only if $f_{1}<\frac{1}{3} I_{13}$ and $f_{3}<\frac{1}{3} I_{13}$. Finally, in $G_{2,3}^{m}(0)$ there exists a NE which strictly Pareto dominates $\left(x_{2}, x_{3}\right)=(0,0)$ if and only if $f_{2}<\frac{1}{3} I_{23}$ and $f_{3}<\frac{1}{3} I_{23}$. Thus, $x=(0,0,0)$ is not a CPNE if at least one of the following sets of conditions is satisfied:

$$
\begin{aligned}
f_{1} & <P_{1}^{d}-P_{1}^{t}(0,0,0) \quad \text { and } f_{2}<P_{2}^{d}-P_{2}^{t}(0,0,0) \\
f_{1} & <\frac{1}{3} I_{13} \text { and } f_{3}<\frac{1}{3} I_{13} \\
f_{2} & <\frac{1}{3} I_{23} \text { and } f_{3}<\frac{1}{3} I_{23}
\end{aligned}
$$

We now maximize $f_{1}+f_{2}+f_{3}$ subject to the constraint (40) and to the constraint that (41)-(43) are all violated and at least one among (44), (45), (46) holds; these constraints makes $x=(1,1,1)$ the unique CPNE. However, it is useful to notice that 
- if (44) holds, then (42) and (43) are both violated;

- if (45) holds, then (41) and (43) are both violated;

- if (46) holds, then (41)-(43) are all violated.

In order to solve this problem we need to consider various values of $M$

The case in which $M$ is between $M^{t}(1,1,1)$ and $M^{t}(1,1,0)$ Let

$$
\tilde{f}_{1} \equiv \min \left\{\frac{1}{2} P_{3}^{t}(0,0,0), \max \left\{\frac{1}{3} I_{13}, \frac{1}{3}\left(I_{12}+I_{13}-2 I_{23}\right)\right\}\right\}
$$

Then in this case the optimal fees are

$$
\begin{aligned}
f_{1} & =\frac{1}{3}\left(I_{12}+I_{13}\right), \quad f_{2}=\frac{1}{3} I_{23}, \quad f_{3}=P_{3}^{t}(1,1,1) \\
\text { if } \frac{1}{3}\left(I_{12}+I_{13}+I_{23}\right)+P_{3}^{t}(1,1,1) & >\tilde{f}_{1}+\frac{1}{2} P_{3}^{t}(0,0,0)+P_{3}^{t}(1,1,1) \\
f_{1} & =\tilde{f}_{1}, \quad f_{2}=\frac{1}{2} P_{3}^{t}(0,0,0), \quad f_{3}=P_{3}^{t}(1,1,1) \\
\text { if } \tilde{f}_{1}+\frac{1}{2} P_{3}^{t}(0,0,0)+P_{3}^{t}(1,1,1) & >\frac{1}{3}\left(I_{12}+I_{13}+I_{23}\right)+P_{3}^{t}(1,1,1)
\end{aligned}
$$

When $M$ is between $M^{t}(1,1,1)$ and $M^{t}(1,1,0)$ we find that $P_{3}^{t}(1,1,1) \leq \frac{1}{3} I_{23}$ and therefore $f_{3}=P_{3}^{t}(1,1,1)$. Now we consider the three ways to satisfy at least one among $(44),(45)$, (46). If the platform wants to satisfy (44), then [in order to violate (41)] it is optimal to $\operatorname{set}^{55} f_{1}=\tilde{f}_{1}, f_{2}=\frac{1}{2} P_{3}^{t}(0,0,0)$ where $P_{1}^{d}-P_{1}^{t}(0,0,0)=P_{2}^{d}-P_{2}^{t}(0,0,0)=\frac{1}{2} P_{3}^{t}(0,0,0)$; thus $R=\tilde{f}_{1}+\frac{1}{2} P_{3}^{t}(0,0,0)+P_{3}^{t}(1,1,1)$. If the platform wants to satisfy $(45)$, then it is optimal to set $f_{1}=\frac{1}{3} I_{13}$; thus $f_{2}=\frac{1}{3}\left(I_{12}+I_{23}\right)$ since (41)-(43) are all violated from $f_{1}=\frac{1}{3} I_{13}$ and $f_{3}<\frac{1}{3} I_{23}$. Hence, $R=\frac{1}{3}\left(I_{12}+I_{13}+I_{23}\right)+P_{3}^{t}(1,1,1)$. If the platform wants to satisfy (46), then it is optimal to set $f_{2}=\frac{1}{3} I_{23}$; thus $f_{1}=\frac{1}{3}\left(I_{12}+I_{13}\right)$ in view of $(40)$ and $R=\frac{1}{3}\left(I_{12}+I_{13}+I_{23}\right)+P_{3}^{t}(1,1,1)$.

In this way we see that for $M$ between $M^{t}(1,1,1)$ and $M^{t}(1,1,0)$, the platform earns $P_{3}^{t}(1,1,1)+\max \left\{\frac{1}{3}\left(I_{12}+I_{13}+I_{23}\right), \tilde{f}_{1}+\frac{1}{2} P_{3}^{t}(0,0,0)\right\}$ by inducing $x=(1,1,1)$, which is higher than the revenue which the platform can make by inducing $x \neq(1,1,1)$. The highest revenue which can be obtained by inducing $x=(1,0,1)$, or $x=(0,1,1)$, or $x=(1,1,0)$ is $\max \left\{\frac{2}{3} I_{13}, P_{3}^{t}(0,0,0)\right\}$. It is clear that $\frac{1}{3}\left(I_{12}+I_{13}+I_{23}\right)>\frac{2}{3} I_{13}$, while $\frac{1}{3}\left(I_{12}+I_{13}+I_{23}\right)$ may be smaller than $P_{3}^{t}(0,0,0)$ only if $I_{12}>2 I_{13}+I_{23}$. But in such a case $\max \left\{\frac{1}{3} I_{23}, \frac{1}{3}\left(I_{12}+I_{13}-2 I_{23}\right)\right\}=\frac{1}{3}\left(I_{12}+I_{13}-2 I_{23}\right)$ and $\tilde{f}_{1}=\frac{1}{3}\left(I_{12}+I_{13}-2 I_{23}\right)$ or $\tilde{f}_{1}=\frac{1}{2} P_{3}^{t}(0,0,0)$. In either case we find that $P_{3}^{t}(1,1,1)+\tilde{f}_{1}+\frac{1}{2} P_{3}^{t}(0,0,0)>P_{3}^{t}(0,0,0)$.

\footnotetext{
${ }^{55}$ The fees in the solution determine the upper bound of the profit the platform can realize. The actual profit can be made arbitrarily close to the bound and this remark applies generally to the optimal fees we characterize.
} 
The case in which $M$ is between $M^{t}(1,1,0)$ and $M^{t}(1,0,1)$ Let

$$
\bar{f}_{1} \equiv \min \left\{\frac{1}{3} I_{13}, \max \left\{\frac{1}{2} P_{3}^{t}(0,0,0), \frac{1}{3}\left(I_{12}+I_{13}-2 I_{23}\right)\right\}\right\}
$$

Then in this case the optimal fees are

$$
\begin{aligned}
& f_{1}=\frac{1}{3}\left(I_{12}+I_{13}\right), \quad f_{2}=\frac{1}{3} I_{23}, \quad f_{3}=\frac{1}{3} I_{23} \\
& \text { if } \frac{1}{3}\left(I_{12}+I_{13}+2 I_{23}\right)>\max \left\{\bar{f}_{1}+\frac{1}{3}\left(I_{12}+I_{23}\right)+P_{3}^{t}(1,1,1), \tilde{f}_{1}+\frac{1}{2} P_{3}^{t}(0,0,0)+P_{3}^{t}(1,1,1)\right\} \\
& f_{1}=\bar{f}_{1}, \quad f_{2}=\frac{1}{3}\left(I_{12}+I_{23}\right), \quad f_{3}=P_{3}^{t}(1,1,1) \\
& \text { if } \bar{f}_{1}+\frac{1}{3}\left(I_{12}+I_{23}\right)+P_{3}^{t}(1,1,1)>\max \left\{\frac{1}{3}\left(I_{12}+I_{13}+2 I_{23}\right), \tilde{f}_{1}+\frac{1}{2} P_{3}^{t}(0,0,0)+P_{3}^{t}(1,1,1)\right\} \\
& f_{1}=\tilde{f}_{1}, \quad f_{2}=\frac{1}{2} P_{3}^{t}(0,0,0), \quad f_{3}=P_{3}^{t}(1,1,1) \\
& \text { if } \tilde{f}_{1}+\frac{1}{2} P_{3}^{t}(0,0,0)+P_{3}^{t}(1,1,1)>\max \left\{\frac{1}{3}\left(I_{12}+I_{13}+2 I_{23}\right), \bar{f}_{1}+\frac{1}{3}\left(I_{12}+I_{23}\right)+P_{3}^{t}(1,1,1)\right\}
\end{aligned}
$$

When $M$ is between $M^{t}(1,1,0)$ and $M^{t}(1,0,1)$ we find that $\frac{1}{3} I_{23}<P_{3}^{t}(1,1,1) \leq \frac{1}{3} I_{13}$ and the platform needs to choose between setting $f_{3}=P_{3}^{t}(1,1,1)$ and $f_{3}=\frac{1}{3} I_{23}$ (actually, slightly smaller). In the first case (46) is violated, and in order to satisfy (45) [and violate (42)] it is necessary to set $f_{1}=\bar{f}_{1}$, then $f_{2}=\frac{1}{3}\left(I_{12}+I_{23}\right)$ and thus $R=\bar{f}_{1}+\frac{1}{3}\left(I_{12}+\right.$ $\left.I_{23}\right)+P_{3}^{t}(1,1,1)$. In alternative, given $f_{3}=P_{3}^{t}(1,1,1)$, the platform can satisfy (44) with $f_{1}=\tilde{f}_{1}, f_{2}=\frac{1}{2} P_{3}^{t}(0,0,0)$, thus $R=\tilde{f}_{1}+\frac{1}{2} P_{3}^{t}(0,0,0)+P_{3}^{t}(1,1,1)$.

In the case of $f_{3}=\frac{1}{3} I_{23}$, the platform can satisfy (46) with $f_{2}=\frac{1}{3} I_{23}$ and then $f_{1}=$ $\frac{1}{3}\left(I_{12}+I_{13}\right) ;$ thus $R=\frac{1}{3}\left(I_{12}+I_{13}+2 I_{23}\right)$. In alternative, the platform can satisfy $(45)$ with $f_{1}=\frac{1}{3} I_{13}$ and then $f_{2}=\frac{1}{3}\left(I_{12}+I_{23}\right)$; thus $R=\frac{1}{3}\left(I_{12}+I_{13}+2 I_{23}\right)$. Finally, the platform can choose $f_{1}=\tilde{f}_{1}$ [in order to violate (41)], $f_{2}=\frac{1}{2} P_{3}^{t}(0,0,0)$ to satisfy (44); then $R=\tilde{f}_{1}+\frac{1}{2} P_{3}^{t}(0,0,0)+\frac{1}{3} I_{23}$.

The revenue which the platform can make is the highest among $\bar{f}_{1}+\frac{1}{3}\left(I_{12}+I_{23}\right)+P_{3}^{t}(1,1,1)$, $\tilde{f}_{1}+\frac{1}{2} P_{3}^{t}(0,0,0)+P_{3}^{t}(1,1,1), \frac{1}{3}\left(I_{12}+I_{13}+2 I_{23}\right)$. It is clear that $\frac{1}{3}\left(I_{12}+I_{13}+2 I_{23}\right)>\frac{2}{3} I_{13}$, and if $\frac{1}{3}\left(I_{12}+I_{13}+2 I_{23}\right)<P_{3}^{t}(0,0,0)$ then $I_{12}>I_{13}+3 I_{23}$, which implies $\max \left\{\frac{1}{3} I_{23}, \frac{1}{3}\left(I_{12}+\right.\right.$ $\left.\left.I_{13}-2 I_{23}\right)\right\}=\frac{1}{3}\left(I_{12}+I_{13}-2 I_{23}\right)$, so that $\tilde{f}_{1}=\frac{1}{3}\left(I_{12}+I_{13}-2 I_{23}\right)$ or $\tilde{f}_{1}=\frac{1}{2} P_{3}^{t}(0,0,0)$. In either case we find that $P_{3}^{t}(1,1,1)+\tilde{f}_{1}+\frac{1}{2} P_{3}^{t}(0,0,0)>P_{3}^{t}(0,0,0)$.

The case in which $M$ is between $M^{t}(1,0,1)$ and $M^{t}(1,0,0) \quad$ In this case the optimal fees are

$$
\begin{aligned}
f_{1} & =\frac{1}{3}\left(I_{12}+I_{13}\right), \quad f_{2}=\frac{1}{3} I_{23}, \quad f_{3}=\frac{1}{3} I_{23} \\
\text { if } \frac{1}{3}\left(I_{12}+I_{13}+2 I_{23}\right) & >\max \left\{\bar{f}_{1}+\frac{1}{3}\left(I_{12}+I_{13}+I_{23}\right), \tilde{f}_{1}+\frac{1}{2} P_{3}^{t}(0,0,0)+P_{3}^{t}(1,1,1)\right\}
\end{aligned}
$$




$$
\begin{gathered}
f_{1}=\bar{f}_{1}, \quad f_{2}=\frac{1}{3}\left(I_{12}+I_{23}\right), \quad f_{3}=\frac{1}{3} I_{13} \\
\text { if } \bar{f}_{1}+\frac{1}{3}\left(I_{12}+I_{13}+I_{23}\right)>\max \left\{\frac{1}{3}\left(I_{12}+I_{13}+2 I_{23}\right), \tilde{f}_{1}+\frac{1}{2} P_{3}^{t}(0,0,0)+P_{3}^{t}(1,1,1)\right\} \\
f_{1}=\tilde{f}_{1}, \quad f_{2}=\frac{1}{2} P_{3}^{t}(0,0,0), \quad f_{3}=P_{3}^{t}(1,1,1) \\
\text { if } \tilde{f}_{1}+\frac{1}{2} P_{3}^{t}(0,0,0)+P_{3}^{t}(1,1,1)>\max \left\{\frac{1}{3}\left(I_{12}+I_{13}+2 I_{23}\right), \bar{f}_{1}+\frac{1}{3}\left(I_{12}+I_{13}+I_{23}\right)\right\}
\end{gathered}
$$

When $M$ is between $M^{t}(1,0,1)$ and $M^{t}(1,0,0)$ we find that $\frac{1}{3} I_{13}<P_{3}^{t}(1,1,1)$. The platform needs to choose among $f_{3}=\frac{1}{3} I_{23}, f_{3}=\frac{1}{3} I_{13}$, and $f_{3}=P_{3}^{t}(1,1,1)$. In the first case it is possible to satisfy (46) with $f_{2}=\frac{1}{3} I_{23}$; thus $f_{1}=\frac{1}{3}\left(I_{12}+I_{13}\right)$ and $R=\frac{1}{3}\left(I_{12}+I_{13}+2 I_{23}\right)$. In alternative, the platform can satisfy (45) with $f_{1}=\frac{1}{3} I_{13}$; then $f_{2}=\frac{1}{3}\left(I_{12}+I_{23}\right)$ and $R=\frac{1}{3}\left(I_{12}+I_{13}+2 I_{23}\right)$. Finally, it is possible to satisfy (44) and violate (41) with $f_{1}=\tilde{f}_{1}$, $f_{2}=\frac{1}{2} P_{3}^{t}(0,0,0)$; thus $R=\frac{1}{3} I_{23}+\tilde{f}_{1}+\frac{1}{2} P_{3}^{t}(0,0,0)$.

In the case of $f_{3}=\frac{1}{3} I_{13},(46)$ is violated and the platform can satisfy (45) and violate (42) with $f_{1}=\bar{f}_{1}$; then $f_{2}=\frac{1}{3}\left(I_{12}+I_{23}\right)$ and $R=\bar{f}_{1}+\frac{1}{3}\left(I_{12}+I_{13}+I_{23}\right)$. In alternative, the platform can satisfy (44) and violate (41) with $f_{1}=\tilde{f}_{1}, f_{2}=\frac{1}{2} P_{3}^{t}(0,0,0)$; thus $R=\tilde{f}_{1}+\frac{1}{2} P_{3}^{t}(0,0,0)+\frac{1}{3} I_{13}$.

In the case of $f_{3}=P_{3}^{t}(1,1,1),(45)-(46)$ are violated and thus it is necessary to choose $f_{1}=$ $\tilde{f}_{1}, f_{2}=\frac{1}{2} P_{3}^{t}(0,0,0)$ to satisfy (44) and violate (41); thus $R=\tilde{f}_{1}+\frac{1}{2} P_{3}^{t}(0,0,0)+P_{3}^{t}(1,1,1)$. Since $P_{3}^{t}(1,1,1)>\frac{1}{3} I_{13}$, the revenue which the platform can make is the highest among $\frac{1}{3}\left(I_{12}+I_{13}+2 I_{23}\right), \bar{f}_{1}+\frac{1}{3}\left(I_{12}+I_{13}+I_{23}\right), \tilde{f}_{1}+\frac{1}{2} P_{3}^{t}(0,0,0)+P_{3}^{t}(1,1,1)$. It is clear that $\frac{1}{3}\left(I_{12}+I_{13}+2 I_{23}\right)>\frac{2}{3} I_{13}$, and if $\frac{1}{3}\left(I_{12}+I_{13}+2 I_{23}\right)<P_{3}^{t}(0,0,0)$ then $I_{12}>I_{13}+2 I_{23}$, which implies $\max \left\{\frac{1}{3} I_{23}, \frac{1}{3}\left(I_{12}+I_{13}-2 I_{23}\right)\right\}=\frac{1}{3}\left(I_{12}+I_{13}-2 I_{23}\right)$, so that $\tilde{f}_{1}=\frac{1}{3}\left(I_{12}+I_{13}-2 I_{23}\right)$ or $\tilde{f}_{1}=\frac{1}{2} P_{3}^{t}(0,0,0)$. In either case we find that $P_{3}^{t}(1,1,1)+\tilde{f}_{1}+\frac{1}{2} P_{3}^{t}(0,0,0)>P_{3}^{t}(0,0,0)$.

11.10.5 When $M^{t}(1,0,0)<M<U-I_{12}$ the optimal fees are $f_{1}=\hat{f}_{1}, f_{2}=\frac{1}{3} I_{12}$, $f_{3}=\frac{1}{3}\left(I_{13}+I_{23}\right)$

Proof. For these values of $M$, we can argue like in step 3 to show that the platform

- can earn $\frac{2}{3} I_{13}$ by inducing $x=(1,0,1)$ with the fees in $(37)$;

- can earn $\frac{2}{3} I_{23}$ by inducing $x=(0,1,1)$ with the fees in $(38)$;

- can earn $\frac{2}{3} I_{12}$ by inducing $x=(1,1,0)$ with the following fees (notice that $x=$ $(1,1,0)$ does not induce 3 to stay inactive)

$$
f_{1}<\frac{1}{3} I_{12}, \quad f_{2}<\frac{1}{3} I_{12}, \quad f_{3} \text { large }
$$


Now we study the revenue the platform can make by inducing $x=(1,1,1)$. This profile is a NE if and only if

$$
f_{1} \leq \frac{1}{3}\left(I_{12}+I_{13}\right), \quad f_{2} \leq \frac{1}{3}\left(I_{12}+I_{23}\right), \quad f_{3} \leq \frac{1}{3}\left(I_{13}+I_{23}\right)
$$

In order to see whether $x=(1,1,1)$ is a CPNE, we study $G_{1,2}^{m}(1), G_{1,3}^{m}(1), G_{2,3}^{m}(1)$ :

$$
\begin{array}{rl|c|c|}
\multicolumn{2}{c}{1 \backslash 2} & \multicolumn{1}{c}{x_{2}=0} & x_{2}=1 \\
\cline { 3 - 4 } G_{1,2}^{m}(1): & x_{1}=0 & P_{1}^{t}(0,0,0), P_{2}^{t}(0,0,0) & P_{1}^{t}(0,0,1), P_{2}^{t}(0,0,1)-f_{2} \\
\cline { 3 - 4 } & x_{1}=1 & P_{1}^{t}(0,1,0)-f_{1}, P_{2}^{t}(0,1,0) & P_{1}^{t}(1,1,1)-f_{1}, P_{2}^{t}(1,1,1)-f_{2} \\
\cline { 3 - 4 } & &
\end{array}
$$

Here $\left(x_{1}, x_{2}\right)=(1,1)$ is a NE, but for some values of $f_{1}, f_{2}$ consistent with (47) we find that $\left(x_{1}, x_{2}\right)=(0,0)$ is a NE and strictly Pareto dominates $\left(x_{1}, x_{2}\right)=(1,1)$. Hence, $x=(1,1,1)$ is not a CPNE if $f_{1} \geq \frac{1}{3} I_{13}, f_{2} \geq \frac{1}{3} I_{23}$ and $f_{1}>\frac{1}{3}\left(I_{12}+I_{13}-2 I_{23}\right)$, $f_{2}>\frac{1}{3}\left(I_{12}+I_{23}-2 I_{13}\right)$.

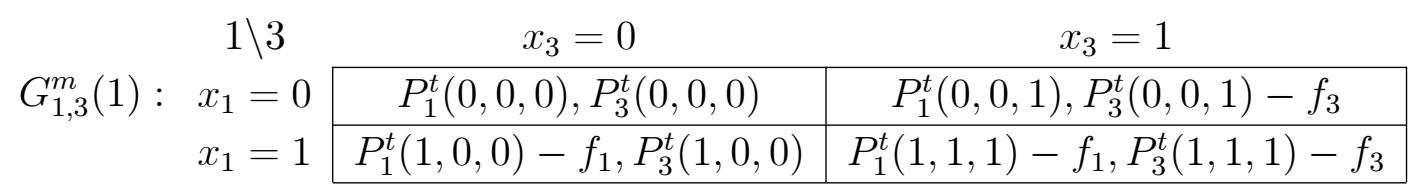

here $\left(x_{1}, x_{3}\right)=(1,1)$ is a NE, but for some values of $f_{1}, f_{3}$ consistent with (47) we find that $\left(x_{1}, x_{3}\right)=(0,0)$ is a NE and strictly Pareto dominates $\left(x_{1}, x_{3}\right)=(1,1)$. Hence, $x=(1,1,1)$ is not a CPNE if $f_{1} \geq \frac{1}{3} I_{12}, f_{3} \geq \frac{1}{3} I_{23}$ and $f_{1}>\frac{1}{3}\left(I_{12}+I_{13}-2 I_{23}\right)$.

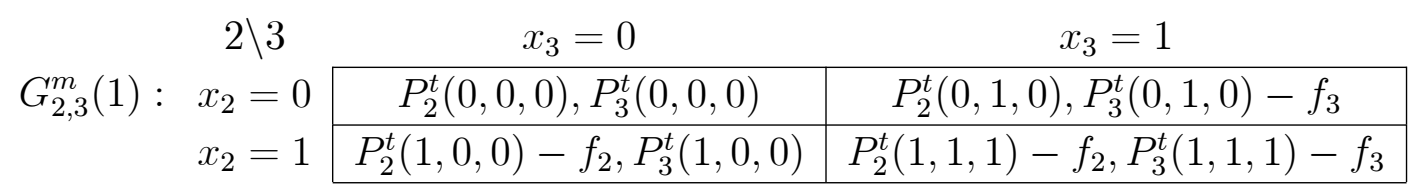

here $\left(x_{2}, x_{3}\right)=(1,1)$ is a NE, but for some values of $f_{2}, f_{3}$ consistent with (47) we find that $\left(x_{2}, x_{3}\right)=(0,0)$ is a $\mathrm{NE}$, and strictly Pareto dominates $\left(x_{2}, x_{3}\right)=(1,1)$. Hence, $x=(1,1,1)$ is not a CPNE if $f_{2} \geq \frac{1}{3} I_{12}, f_{3} \geq \frac{1}{3} I_{13}$ and $f_{2}>\frac{1}{3}\left(I_{12}+I_{23}-2 I_{13}\right)$, but the latter inequality is satisfied when the first inequality holds.

Therefore, $x=(1,1,1)$ is not a CPNE if at least one of the three sets of conditions (48)-(50) below is satisfied. Equivalently, $x=(1,1,1)$ is a CPNE if and only if the following three sets of conditions are all violated [and (47) is satisfied]

$$
\begin{array}{ll}
f_{1} \geq \frac{1}{3} I_{13}, \quad f_{1}>\frac{1}{3}\left(I_{12}+I_{13}-2 I_{23}\right) & \text { and } \quad f_{2} \geq \frac{1}{3} I_{23}, \quad f_{2}>\frac{1}{3}\left(I_{12}+I_{23}-2 I_{13}(48)\right. \\
f_{1} \geq \frac{1}{3} I_{12}, \quad f_{1}>\frac{1}{3}\left(I_{12}+I_{13}-2 I_{23}\right) & \text { and } \quad f_{3} \geq \frac{1}{3} I_{23} \\
f_{2} \geq \frac{1}{3} I_{12} & \text { and } \quad f_{3} \geq \frac{1}{3} I_{13}
\end{array}
$$


Furthermore, for some fees which violate (48)-(50) we have that $x=(0,0,0)$ is a CPNE. Precisely, $x=(0,0,0)$ is always a NE and we need to study $G_{1,2}^{m}(0), G_{1,3}^{m}(0), G_{2,3}^{m}(0)$ to establish whether it is also a CPNE

$$
\begin{aligned}
& \begin{array}{ccccc|} 
& \multicolumn{1}{c}{1 \backslash 2} & \multicolumn{2}{c}{x_{2}=0} & x_{2}=1 \\
\cline { 3 - 4 } G_{1,2}^{m}(0): & x_{1}=0 & P_{1}^{t}(0,0,0), P_{2}^{t}(0,0,0) & P_{1}^{t}(0,0,0), P_{2}^{t}(0,0,0)-f_{2} \\
\cline { 3 - 4 } & x_{1}=1 & P_{1}^{t}(0,0,0)-f_{1}, P_{2}^{t}(0,0,0) & P_{1}^{t}(1,0,0)-f_{1}, P_{2}^{t}(1,0,0)-f_{2} \\
\cline { 3 - 4 } & & &
\end{array} \\
& 1 \backslash 3 \quad x_{3}=0 \quad x_{3}=1 \\
& \begin{array}{ccc|c|c|}
G_{1,3}^{m}(0): & x_{1}=0 & P_{1}^{t}(0,0,0), P_{3}^{t}(0,0,0) & P_{1}^{t}(0,0,0), P_{3}^{t}(0,0,0)-f_{3} \\
\cline { 3 - 4 } & x_{1}=1 & P_{1}^{t}(0,0,0)-f_{1}, P_{3}^{t}(0,0,0) & P_{1}^{t}(0,1,0)-f_{1}, P_{3}^{t}(0,1,0)-f_{3} \\
\cline { 3 - 4 } & &
\end{array}
\end{aligned}
$$

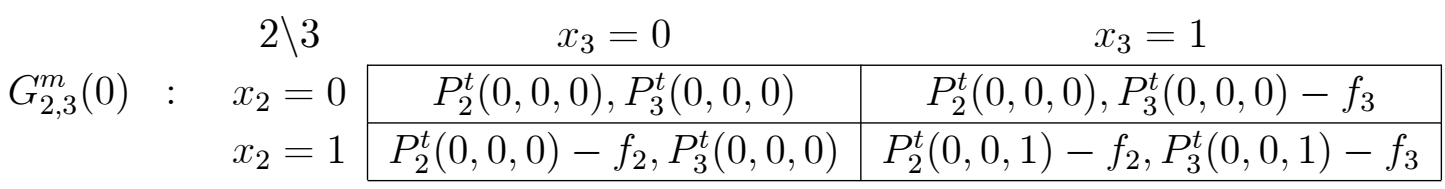

By arguing as in step 4 we see that $x=(0,0,0)$ is not a CPNE if and only if at least one of the following conditions is satisfied:

$$
\begin{aligned}
f_{1} & <\frac{1}{3} I_{12} & \text { and } & f_{2} & <\frac{1}{3} I_{12} \\
f_{1} & <\frac{1}{3} I_{13} & \text { and } & f_{3} & <\frac{1}{3} I_{13} \\
f_{2} & <\frac{1}{3} I_{23} & \text { and } & f_{3} & <\frac{1}{3} I_{23}
\end{aligned}
$$

Therefore we maximize $f_{1}+f_{2}+f_{3}$ subject to the constraint (47) and to the constraint that (48)-(50) are all violated, and at least one among (51), (52), (53) is satisfied. However, it is useful to notice that

- if (51) holds, then (49)-(50) are both violated;

- if (52) holds, then (48)-(50) are all violated;

- if (53) holds, then (48)-(50) are all violated.

In order to solve this problem, we consider the three ways to satisfy at least one condition among (51), (52), (53). If the platform wants to satisfy (51), then it is optimal to set

$$
f_{1}=\hat{f}_{1} \equiv \min \left\{\frac{1}{3} I_{12}, \max \left\{\frac{1}{3} I_{13}, \frac{1}{3}\left(I_{12}+I_{13}-2 I_{23}\right)\right\}\right\}
$$

[in order to violate (48)], $f_{2}=\frac{1}{3} I_{12}$; thus $f_{3}=\frac{1}{3}\left(I_{13}+I_{23}\right)$ in view of (47). Then, the revenue is $R=\hat{f}_{1}+\frac{1}{3}\left(I_{12}+I_{13}+I_{23}\right)$. If the platform wants to satisfy (52), then it is optimal to set $f_{1}=\frac{1}{3} I_{13}, f_{3}=\frac{1}{3} I_{13}$; thus $f_{2}=\frac{1}{3}\left(I_{12}+I_{23}\right)$ in view of $(47)$ and $R=\frac{1}{3}\left(I_{12}+2 I_{13}+I_{23}\right)$. If the platform wants to satisfy (53), then it is optimal to set $f_{2}=\frac{1}{3} I_{23}, f_{3}=\frac{1}{3} I_{23}$; thus 
$f_{1}=\frac{1}{3}\left(I_{12}+I_{13}\right)$ in view of $(47)$ and $R=\frac{1}{3}\left(I_{12}+I_{13}+2 I_{23}\right)$. Since $\hat{f}_{1} \geq \frac{1}{3} I_{13}$, we conclude that $\hat{f}_{1}+\frac{1}{3}\left(I_{12}+I_{13}+I_{23}\right)$ is the highest among the three revenues. Moreover, we can prove that $\hat{f}_{1}+\frac{1}{3}\left(I_{12}+I_{13}+I_{23}\right)$ is also higher than $\frac{2}{3} I_{12}$, the highest revenue which can be obtained by inducing $x=(1,0,1)$, or $x=(0,1,1)$, or $x=(1,1,0)$ : (i) if $\hat{f}_{1}=\frac{1}{3} I_{12}$, then it is obvious that $\hat{f}_{1}+\frac{1}{3}\left(I_{12}+I_{13}+I_{23}\right)>\frac{2}{3} I_{12}$; (ii) if $\hat{f}_{1}=\frac{1}{3}\left(I_{12}+I_{13}-2 I_{23}\right)$, then $\hat{f}_{1}+\frac{1}{3}\left(I_{12}+I_{13}+I_{23}\right)-\frac{2}{3} I_{12}=\frac{1}{3}\left(2 I_{13}-I_{23}\right)>0$; (iii) if $\hat{f}_{1}=\frac{1}{3} I_{13}$, then $I_{12}<2 I_{23}$ and $\hat{f}_{1}+\frac{1}{3}\left(I_{12}+I_{13}+I_{23}\right)-\frac{2}{3} I_{12}=\frac{1}{3}\left(2 I_{13}+I_{23}-I_{12}\right)>0$. Hence, the optimal fees are $f_{1}=\hat{f}_{1}$, $f_{2}=\frac{1}{3} I_{12}, f_{3}=\frac{1}{3}\left(I_{13}+I_{23}\right)$.

\subsection{Proof of Corollary 2}

We first prove a result, called Step 0, and then prove (i) and (ii)

Step 0 For each publisher $i, x_{i}=1$ weakly dominates $x_{i}=0$ in $\hat{G}^{m}$ Proof. Step 1 in the proof of Proposition 2 for the general version establishes that $x_{i}=1$ weakly dominates $x_{i}=0$ in the market for library $h$, that is $\pi_{i}^{h}\left(x_{i}=1, x_{j}, x_{k}\right) \geq \pi_{i}^{h}\left(x_{i}=0, x_{j}, x_{k}\right)$ for $h=1, \ldots, n$. Since $\pi_{i}(x)=\pi_{i}^{1}(x)+\ldots+\pi_{i}^{n}(x)$, it follows that $x_{i}=1$ weakly dominates $x_{i}=0$ in $\hat{G}^{m}$, for each publisher $i$.

\section{Proof of Corollary 2(i)}

This proof is split into two steps

Step $1 x=(0,0,0)$ is not a CPNE of $\hat{G}^{m} \quad$ Proof. Without loss of generality, suppose that market $n$ is such that $M^{n}>M^{t, n}(1,1,1)$ and $U_{i}^{n}>U_{j}^{n}>U_{k}^{n}>0$. We prove that $\left(x_{i}, x_{j}\right)=(1,1)$ is a NE of $\hat{G}_{i, j}^{m}(0)$ and is a Pareto improvement for $i$ and $j$. Indeed, step 0 implies that $\left(x_{i}, x_{j}\right)=(1,1)$ is a NE of $\hat{G}_{i, j}^{m}(0)$ and we need to prove that $\pi_{i}\left(x_{i}=1, x_{j}=\right.$ $\left.1, x_{k}=0\right)>\pi_{i}(0,0,0)$ and $\pi_{j}\left(x_{i}=1, x_{j}=1, x_{k}=0\right)>\pi_{j}(0,0,0)$. We start by proving that $\pi_{i}^{n}\left(x_{i}=1, x_{j}=1, x_{k}=0\right)>\pi_{i}^{n}(0,0,0)$ and $\pi_{j}^{n}\left(x_{i}=1, x_{j}=1, x_{k}=0\right)>\pi_{j}^{n}(0,0,0)$, that is both publishers $i$ and $j$ increase their profits in market $n$ by getting interconnected. Indeed, this makes the profit of firm $i$ in market $n$ equal to either $P_{i, j}^{d, n}$ or $P_{i}^{t, n}\left(y_{i j}=1, y_{i k}=\right.$ $\left.y_{j k}=0\right)$, and both these terms are strictly larger than $P_{i}^{t, n}(0,0,0)$, the profit of $i$ with $y=(0,0,0)$. Furthermore, for any $h \neq n, \pi_{i}^{h}\left(x_{i}=1, x_{j}=1, x_{k}=0\right) \geq \pi_{i}^{h}\left(x_{i}=0, x_{j}=\right.$ $\left.1, x_{k}=0\right)$ holds by step 0 and $\pi_{i}^{h}\left(x_{i}=0, x_{j}=1, x_{k}=0\right)=\pi_{i}^{h}(0,0,0)$ because the interconnection of a single publisher has no effect. Since $\pi_{i}(x)=\pi_{i}^{1}(x)+\ldots+\pi_{i}^{n}(x)$, our result is obtained.

Step $2 x=(1,1,1)$ is the unique CPNE Proof. Step 0 implies that $x=(1,1,1)$ is a NE in $\hat{G}^{m}$. In order to verify that it is also a CPNE, consider $\hat{G}_{i, j}^{m}(1)$ for arbitrary $i, j$ and notice that for $h$ such that $M^{h}>M^{t, h}(1,1,1), x_{i}=1$ strictly dominates $x_{i}=0$ for 
publisher $i$ in market $h$, given that $x_{k}=1: \pi_{i}^{h}\left(x_{i}=1, x_{j}, x_{k}=1\right)>\pi_{i}^{h}\left(x_{i}=0, x_{j}, x_{k}=1\right)$. For $h$ such that $M^{h} \leq M^{t, h}(1,1,1), x_{i}=1$ weakly dominates $x_{i}=0$ for publisher $i$ in market $h: \pi_{i}^{h}\left(x_{i}=1, x_{j}, x_{k}=1\right) \geq \pi_{i}^{h}\left(x_{i}=0, x_{j}, x_{k}=1\right)$. Thus, $x_{i}=1$ strictly dominates $x_{i}=0$ for publisher $i$ in $\hat{G}^{m}$ if $x_{j}=1$ and/or $x_{k}=1$; hence $x_{i}=1$ is strictly dominant in $\hat{G}_{i, j}^{m}(1)$. It follows that $\left(x_{i}, x_{j}\right)=(1,1)$ is the unique NE of $\hat{G}_{i, j}^{m}(1)$ and that $x=(1,1,1)$ is a CPNE in $\hat{G}^{m}$.

We prove uniqueness as in the proof of Proposition 2: Step 1 rules out that $x=(0,0,0)$ is a CPNE, thus at least one publisher interconnects in any CPNE, and therefore all interconnect because of strict dominance.

\section{Proof of Corollary 2 (ii)}

This proof is split into two steps

Step $1 x=(1,1,1)$ is a CPNE Proof. Step 0 implies that $x=(1,1,1)$ is a NE in $\hat{G}^{m}$. In order to prove that it is a CPNE, we consider any two publishers $i, j$ and $\hat{G}_{i, j}^{m}(1)$. Clearly, $\left(x_{i}, x_{j}\right)=(1,1)$ is a $\mathrm{NE}$ in this game and we prove that there exists no Pareto improving NE by examining the other profiles in $\hat{G}_{i, j}^{m}(1):\left(x_{i}, x_{j}\right)=(1,0),\left(x_{i}, x_{j}\right)=(0,0)$ and $\left(x_{i}, x_{j}\right)=(0,1)$. Without loss of generality, we suppose $U_{i}^{h}>U_{j}^{h}$. Then, there are four possible cases to consider: $U_{i}^{h}>U_{j}^{h}>U_{k}^{h}, U_{i}^{h}>U_{k}^{h}>U_{j}^{h}$ and $U_{k}^{h}>U_{i}^{h}>U_{j}^{h}$.

- $\left(x_{i}, x_{j}\right)=(1,0)$ implies $y_{i k}=1, y_{i j}=y_{j k}=0$. If a market $h$ is such that $U_{i}^{h}>$ $U_{j}^{h}>U_{k}^{h}$, then $y_{i k}=1, y_{i j}=y_{j k}=0$ induces the same outcome as $y=(1,1,1)$ if $M^{h} \leq \max \left\{U_{i}^{h}-U_{k}^{h}, M^{t, h}\left(y_{i k}=1, y_{i j}=y_{j k}=0\right)\right\}$ (by Lemmas 3 and 8) and induces a different outcome otherwise. However, this different outcome yields publisher $j$ profit $P_{j}^{t, h}(y)$ or 0 , which is smaller than his profit $P_{j, i}^{d, h}$ when $y=(1,1,1)$ [recall that $M^{h} \leq M^{t, h}(1,1,1)$ for any $h$; hence, $j$ increases his profit in market $h$ by playing $x_{j}=1$. Since $x_{j}=1$ weakly dominates $x_{j}=0$ in every market, we infer that $\left(x_{i}, x_{j}\right)=(1,0)$ is not a NE in $\hat{G}_{i, j}^{m}(1)$ if there is a market $h$ such that $U_{i}^{h}>U_{j}^{h}>U_{k}^{h}$ in which it generates a different outcome with respect to $\left(x_{i}, x_{j}\right)=(1,1)$.

When we consider a market $h$ such that $U_{i}^{h}>U_{k}^{h}>U_{j}^{h}$ or $U_{k}^{h}>U_{i}^{h}>U_{j}^{h}$, we see that $\left(x_{i}, x_{j}\right)=(1,0)$ always generates the same outcome as $\left(x_{i}, x_{j}\right)=(1,1)$ [recall that $M^{h} \leq M^{t, h}(1,1,1)$ for any $h$ ], and thus it cannot generate a Pareto improvement for publishers $i$ and $j$ with respect to $\left(x_{i}, x_{j}\right)=(1,1)$.

- $\left(x_{i}, x_{j}\right)=(0,0)$ implies $y=(0,0,0)$. If a market $h$ is such that $U_{i}^{h}>U_{j}^{h}>U_{k}^{h}$, then $y=(0,0,0)$ induces the same outcome as $y=(1,1,1)$ if $M^{h} \leq M^{t, h}(0,0,0)$, and induces a different outcome otherwise. However, this different outcome yields publisher $i$ a profit of $P_{i}^{t, h}(0,0,0)$, which is smaller than his profit $P_{i, k}^{d, h}$ or $P_{i}^{t, h}\left(y_{i k}=\right.$ $1, y_{i j}=y_{j k}=0$ ) when he plays $x_{i}=1$ (see Lemma 8); thus, $i$ increases his profit in market $h$ by playing $x_{i}=1$. Since $x_{i}=1$ weakly dominates $x_{i}=0$ in every 
market, we infer that $\left(x_{i}, x_{j}\right)=(0,0)$ is not a NE in $\hat{G}_{i, j}^{m}(1)$ if there is a market $h$ such that $U_{i}^{h}>U_{j}^{h}>U_{k}^{h}$ in which it generates a different outcome with respect to $\left(x_{i}, x_{j}\right)=(1,1)$. When we consider a market $h$ such that $U_{i}^{h}>U_{k}^{h}>U_{j}^{h}$ or $U_{k}^{h}>U_{i}^{h}>U_{j}^{h}$, an argument similar to the above one applies, but relies on Lemma 3 and not on Lemma 8.

- $\left(x_{i}, x_{j}\right)=(0,1)$ implies $y_{j k}=1, y_{i j}=y_{i k}=0$. If a market $h$ is such that $U_{i}^{h}>$ $U_{j}^{h}>U_{k}^{h}$, then $y_{j k}=1, y_{i j}=y_{i k}=0$ may induce a different outcome with respect to $y=(1,1,1)$ (see Lemma 11) for $M^{h} \leq U_{i}^{h}-U_{j}^{h}$ if $A^{h}=\{j, k\}$ instead of $A^{h}=\{i\}$, but $i$ can profitably deviate with $x_{i}=1$ and earn $M^{h}$ instead of 0 ; or for $U_{i}^{h}-U_{j}^{h}<M^{h} \leq M^{t, h}(1,1,1)$ if $A^{h}=\{j, k\}$ or $A^{h}=\{1,2,3\}$ instead of $A^{h}=\{i, j\}$, but $i$ can profitably deviate with $x_{i}=1$ and increase his profit as $P_{i, j}^{d, h}>0$ and $P_{i, j}^{d, h}>P_{i}^{t, h}\left(y_{j k}=1, y_{i j}=y_{i k}=0\right)$. When we consider a market $h$ such that $U_{i}^{h}>U_{k}^{h}>U_{j}^{h}$, we can argue like when $U_{i}^{h}>U_{j}^{h}>U_{k}^{h}$. If a market $h$ is such that $U_{k}^{h}>U_{i}^{h}>U_{j}^{h}$, we can again prove that in case that $\left(x_{i}, x_{j}\right)=(0,1)$ induces a different outcome with respect to $\left(x_{i}, x_{j}\right)=(1,1)$, then $i$ can profitably deviate by playing $x_{i}=1$ [the argument relies on Lemmas 3 and 8 like above in the proof of this step].

Step 2 Any CPNE yields the same outcome as $x=(1,1,1)$ Proof. Let us start by considering $x=(0,0,0)$. For any market $h$ such that $M^{h} \leq M^{t, h}(0,0,0)$, the outcome in market $h$ is exactly like when $x=(1,1,1)$. However, if $M^{h}>M^{t, h}(0,0,0)$ for at least one $h$, then all publishers are active in that market when $x=(0,0,0)$, while only the two largest publishers, $1^{h}$ and $2^{h}$ are active in that market if $x=(1,1,1)$. By arguing like in step 1 in the proof of part (1) of this corollary we can prove that $x=(0,0,0)$ is not a CPNE.

Now let us consider $x=(1,0,0)$. This profile of interconnection implies $y=(0,0,0)$ like when $x=(0,0,0)$, and indeed we can argue almost exactly like above to show that $x=(1,0,0)$ is not a CPNE if $M^{h}>M^{t, h}(0,0,0)$ for at least one $h$. If instead $M^{h} \leq M^{t, h}(0,0,0)$ for any $h$, then the outcome in each market is like when $x=(1,1,1)$. In a similar way we deal with $x=(0,1,0)$ and $x=(0,0,1)$.

About $x=(1,1,0), x=(1,0,1), x=(0,1,1)$, we argue like in step 1 in the proof of part (ii) of this corollary to show that whenever there is at least one market in which the outcome is different with respect to the outcome with $x=(1,1,1)$, then the (unique) firm which is not interconnected has a strict incentive to deviate by interconnecting. 


\subsection{Proof of Proposition 6}

(i) This result is a corollary of Proposition 2(ii), given that $M^{t}(1,1,1)=0$ under symmetry.

(ii) The proof of this part is organized in four steps.

Step 1 For any $M$ between 0 and $U-I$, there is no $\mathbf{x}$ which is CPNE and such that $y=(0,0,0)$.

Proof. Let $\mathbf{x}$ be such that $y=(0,0,0)$. First, if $x_{i j}=1$ for some publishers $i, j$, then $\mathbf{x}$ is not a NE of $G^{b}$. Indeed, it must be the case that $x_{j i}=0$ in order to obtain $y_{i j}=0$, but if $j$ deviates with $x_{j i}=1, x_{j k}=0$ then $y_{i j}=1, y_{i k}=y_{j k}=0$ and then $j$ earns $\frac{M}{2}$ if $M \leq 2 I$ (as publisher $k$ is not active) or $\frac{M+I}{3}$ if $M>2 I$ (as publisher $k$ is active). Since $\frac{M}{2}$ and $\frac{M+I}{3}$ are larger than $\frac{M}{3}, j$ 's profit when $y=(0,0,0)$, we infer that $j$ 's deviation is profitable. Therefore we need to consider only the case of $\mathbf{x}_{1}=\mathbf{x}_{2}=\mathbf{x}_{3}=(0,0)$, which is a NE of $G^{b}$. However, $\mathbf{x}_{1}=\mathbf{x}_{2}=\mathbf{x}_{3}=(0,0)$ is not a CPNE because $\mathbf{x}_{1}=\mathbf{x}_{2}=(1,0)$ is $\mathrm{NE}$ in $G_{1,2}^{b}(0,0)$ and the profit of both 1 and 2 is either $\frac{M}{2}$ (if $M \leq 2 I$ ) or $\frac{M+I}{3}$ (if $M>2 I$ ), which are both higher than $\frac{M}{3}$, the profit of 1 and 2 with $\mathbf{x}_{1}=\mathbf{x}_{2}=\mathbf{x}_{3}=(0,0)$.

Step 2 For any $M$ between 0 and $I$, any CPNE is such that $A^{*}=\{1,2\}$ and $P_{1}=$ $P_{2}=\frac{M}{2}$; for instance, $x_{1}=x_{2}=(1,0), x_{3}=(0,0)$ is a CPNE.

Proof. First, having excluded $y=(0,0,0)$, when $M \leq I$ the three publishers are all active if and only if $y=(1,1,1)$, which occurs if and only if $\mathbf{x}_{1}=\mathbf{x}_{2}=\mathbf{x}_{3}=(1,1)$. However, $\mathbf{x}_{1}=\mathbf{x}_{2}=\mathbf{x}_{3}=(1,1)$ is not a CPNE because each publisher makes a profit $\frac{M}{3}$, but $\mathbf{x}_{1}=\mathbf{x}_{2}=(1,0)$ is a NE in $G_{1,2}^{b}(1,1)$ and in this NE both 1 and 2 make a profit $\frac{M}{2}>\frac{M}{3}$.

Hence, only 1 and 2 are active and $\mathbf{x}_{1}=\mathbf{x}_{2}=(1,0), \mathbf{x}_{3}=(0,0)$ is a CPNE which induces this outcome, through the interconnection profile $y=(1,0,0)$. Precisely, with $\mathbf{x}_{1}=\mathbf{x}_{2}=(1,0), \mathbf{x}_{3}=(0,0)$ publishers 1 and 2 earn $\frac{M}{2}$ each, and it is simple to see that this profile is a NE since $\frac{M}{2}>\frac{M}{3}$. This profile is also a CPNE because

- In $G_{1,2}^{b}(1,0)$ it is infeasible that 1 and 2 both earn more than $\frac{M}{2}$, since in aggregate the profits of the publishers are equal to $M$.

- In $G_{1,3}^{b}(1,0)$ it is infeasible that 1 and 3 both increase their profits above $\frac{M}{2}$ and 0 respectively. That requires that both 1 and 3 are active, and if $A=\{1,3\}$ then each of them makes a profit equal to $\frac{M}{2}$, so that the profit of 1 is not increased. If instead all the three publishers are active, since $M \leq I$ and $x_{23}=0$ imply $y_{23}=0$, it is necessary that $y=(0,0,0)$, and thus 1 's profit is $\frac{M}{3}\left(<\frac{M}{2}\right)$.

- In $G_{2,3}^{b}(1,0)$ it is infeasible that 2 and 3 both increase their profits above $\frac{M}{2}$ and 0 respectively, by the same arguments given for $G_{1,3}^{b}(1,0)$. 
There also exists a CPNE which induces the interconnection profile $y=(1,1,0)$, such as $\mathbf{x}_{1}=(1,1), \mathbf{x}_{2}=\mathbf{x}_{3}=(1,0)$. In this CPNE only 1 and 2 are active, so that each of them earns $\frac{M}{2}$. In order to see that $\mathbf{x}_{1}=(1,1), \mathbf{x}_{2}=\mathbf{x}_{3}=(1,0)$ is a CPNE we can argue exactly like above.

Step 3 For any $M>I$ the profile $\mathbf{x}_{1}=\mathbf{x}_{2}=\mathbf{x}_{3}=(1,1)$ is a CPNE

Proof. When $\mathbf{x}_{1}=\mathbf{x}_{2}=\mathbf{x}_{3}=(1,1)$, we find that $y=(1,1,1)$ : all publishers are active and each of them gains $\frac{M}{3}$. In order to see that $\mathbf{x}_{1}=\mathbf{x}_{2}=\mathbf{x}_{3}=(1,1)$ is a NE, notice that if publisher 1 (i) plays $\mathbf{x}_{1}=(0,0)$, it gains $0<\frac{M}{3}$ if $M \leq 2 I$ or $\frac{M-2 I}{3}$ if $M>2 I$; (ii) plays $\mathbf{x}_{1}=(1,0)$ or $\mathbf{x}_{1}=(0,1)$, it gains $\frac{M-I}{3}<\frac{M}{3}$. In order to see that $\mathbf{x}_{1}=\mathbf{x}_{2}=\mathbf{x}_{3}=(1,1)$ is a CPNE, we examine $G_{1,2}^{b}(1,1)$ :

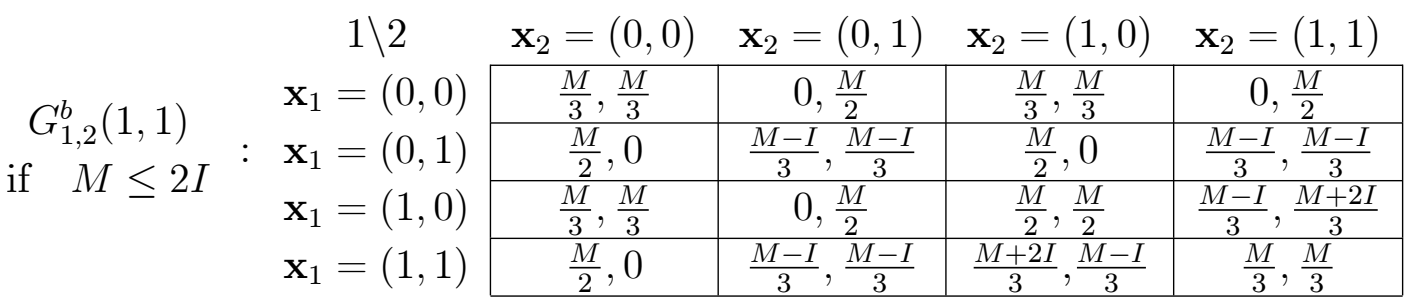

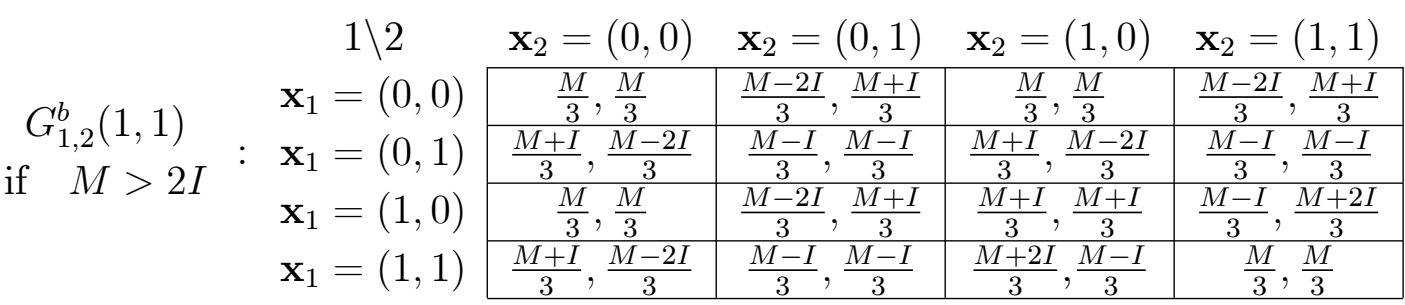

It is simple to see that (in both cases), in $G_{1,2}^{b}(1,1)$ the only $\mathbf{x}_{1}^{\prime}, \mathbf{x}_{2}^{\prime}$ which 1 and 2 both prefer to $\mathbf{x}_{1}=\mathbf{x}_{2}=(1,1)$ is $\mathbf{x}_{1}^{\prime}=\mathbf{x}_{2}^{\prime}=(1,0)$. But $\mathbf{x}_{1}^{\prime}=\mathbf{x}_{2}^{\prime}=(1,0)$ is not a $\mathrm{NE}$ of $G_{1,2}^{b}(1,1)-1$ prefers to play $(1,1)$ if 2 plays $\mathbf{x}_{2}^{\prime}-$ and thus there exists no NE in $G_{1,2}^{b}(1,1)$ which 1 and 2 both prefer to $\mathbf{x}_{1}=\mathbf{x}_{2}=(1,1)$. Furthermore, $G_{1,3}^{b}(1,1)$ and $G_{2,3}^{b}(1,1)$ are isomorphic to $G_{1,2}^{(1,1)}$, and so we get the same result.

Step 4 For any $M>I$ there is no CPNE such that $y=(1,0,0)$ or $y=(1,1,0)$ Proof. If $y=(1,1,0)$, then $\mathbf{x}_{1}=(1,1)$ and in $G_{2,3}^{b}(1,1)$ there is a NE such that $\mathbf{x}_{2}=\mathbf{x}_{3}=(1,1)$ and 2 and 3 both obtain profit $\frac{M}{3}$, which is higher than $\frac{M-I}{3}$, the profit 2 and 3 both make when $y=(1,1,0)$.

If $y=(1,0,0)$, then it is necessary that $x_{13}=x_{31}=x_{23}=x_{32}=0$ because $x_{31}=1$ (for instance) implies that 1 wants to play $x_{13}=1$ since $\frac{M+2 I}{3}>\frac{M}{2}$ (for $M \leq 2 I$ ) and $\frac{M+2 I}{3}>\frac{M+I}{3}($ for $M>2 I)$; if $x_{23}=1$, then 3 wants to deviate to $x_{32}=1$ since $\frac{M-I}{3}>$ $\max \left\{0, \frac{M-2 I}{3}\right\}$. The only remaining profile such that $y=(1,0,0)$ is $\mathbf{x}_{1}=\mathbf{x}_{2}=(1,0)$, $\mathbf{x}_{3}=(0,0)$, but we find that $\mathbf{x}_{1}=(1,1), \mathbf{x}_{3}=(1,0)$ is a NE in $G_{1,3}^{b}(1,0)$, and then all three publishers are active, 1 obtains $\frac{M+2 I}{3}$ and 3 obtains $\frac{M-I}{3}$. 